\title{
Low-lying even parity meson resonances and spin-flavor symmetry
}

\author{
C. García-Recio \\ Departamento de Física Atómica, Molecular y Nuclear, \\ Universidad de Granada, E-18071 Granada, Spain \\ L.S. Geng \\ School of Physics and Nuclear Energy Engineering, Beihang University, Beijing 100191, China and \\ Physik Department, Technische Universität München, D-85747 Garching, Germany \\ J. Nieves \\ Instituto de Física Corpuscular (IFIC), Centro Mixto CSIC-Universidad de Valencia, \\ Institutos de Investigación de Paterna, Aptd. 22085, E-46071 Valencia, Spain \\ L.L. Salcedo \\ Departamento de Física Atómica, Molecular y Nuclear, \\ Universidad de Granada, E-18071 Granada, Spain
}

(Dated: November 16, 2018)

\begin{abstract}
Based on a spin-flavor extension of chiral symmetry, a novel $s$-wave meson-meson interaction involving members of the $\rho$-nonet and of the $\pi$-octet is introduced and its predictions are analyzed. The starting point is the $\mathrm{SU}(6)$ version of the $\mathrm{SU}(3)$ flavor Weinberg-Tomozawa Lagrangian. SU(6) symmetry breaking terms are then included to account for the physical meson masses and decay constants in a way that preserves (broken) chiral symmetry. Next, the $T$-matrix amplitudes are obtained by solving the Bethe Salpeter equation in coupled-channel and the poles are identified with their possible Particle Data Group (PDG) counterparts. It is shown that most of the low-lying even parity PDG meson resonances, specially in the $J^{P}=0^{+}$and $1^{+}$sectors, can be classified according to multiplets of $\mathrm{SU}(6)$. The $f_{0}(1500), f_{1}(1420)$ and some $0^{+}\left(2^{++}\right)$resonances cannot be accommodated within this scheme and thus they would be clear candidates to be glueballs or hybrids. Finally, we predict the existence of five exotic resonances $(I \geq 3 / 2$ and/or $|Y|=2)$ with masses in the range 1.4-1.6 GeV, which would complete the $27_{1}, 10_{3}$, and $10_{3}^{*}$ multiplets of $\mathrm{SU}(3) \otimes \mathrm{SU}(2)$.
\end{abstract}

PACS numbers: 11.10.St Bound and unstable states; Bethe-Salpeter equations, 13.75.Lb Mes14.40.Rt Exotic mesons on-Meson interactions, 14.40.Be Light mesons $(\mathrm{S}=\mathrm{C}=\mathrm{B}=0)$, 14.40.Rt Exotic mesons.

\section{INTRODUCTION}

Chiral perturbation theory (ChPT), a systematic implementation of chiral symmetry and of its pattern of spontaneous and explicit breaking, provides a model independent scheme where multitude of low-energy non-perturbative strong-interaction phenomena can be understood. It has been successfully applied to study different processes, both in the meson-meson and in the meson-baryon sectors, involving light $(u$ and $d)$ or strange $(s)$ quarks [1 10 ].

However, by construction, ChPT is only valid at low-energies and it cannot describe the nature of hadron resonances. In recent years, it has been shown that by unitarizing the ChPT amplitudes one can greatly extend the region of application of ChPT. ${ }^{1}$ This approach, commonly referred as Unitary Chiral Perturbation Theory (UChPT), has received much attention and provided many interesting results [1] 46]. In particular, many meson-meson and mesonbaryon resonances and bound states appear naturally within UChPT. These states are then interpreted as having "dynamical nature." In other words, they are not genuine $q \bar{q}$ or $q q q$ states, but are mainly built out of their mesonmeson or meson-baryon components. One way to distinguish these two pictures is to study the dependence on $N_{C}$ of the resonance masses and widths [47 54].

Some examples are the low-lying scalar mesons, $f_{0}(600), f_{0}(980), a_{0}(980)$ and $K_{0}^{*}(800)$, which naturally appear as resonant states of two mesons of the pion octet [15, 18 22, 24, 27, 30], or the low-lying $J^{P}=1 / 2^{-}$baryonic resonances, $N(1535), N(1650), \Lambda(1405)$ and $\Lambda(1670)$, which are found after unitarizing the ChPT amplitudes for the scattering of $\pi$ pseudoscalar octet mesons off baryons belonging to nucleon octet [14, 17, 28, 31 34, 36, 38, 41, 42], or the low-lying

\footnotetext{
${ }^{1}$ Several frameworks have been proposed to unitarize the amplitudes, though the most common and successful are the inverse amplitude method and the solution of the Bethe-Salpeter (BS) equation. In this latter case, several renormalization schemes have been also employed, differing mostly in the treatment of the off-shell effects. In general, the different methods give similar results for the lowestlying resonances.
} 
$J^{P}=3 / 2^{-}$baryonic resonances (e.g. the $\Lambda(1520)$ ) found in the interaction of mesons of the pion octet with baryons of the $\Delta$ decuplet [35, 40, 46], and finally the low-lying axial vector mesons, $a_{1}(1260), b_{1}(1235), h_{1}(1170), f_{1}(1285)$, $K_{1}(1270)$ that can be described as resonant states of a $\pi$ octet and a $\rho$ vector nonet mesons [37, 43].

These ideas have also been extended to study three-body meson resonances 55, 56] and to systems including a heavy quark, which has allowed to describe also meson [57 60] and baryon [61 66] charmed resonances. In these latter cases, of course one cannot invoke chiral dynamics to construct the tree level amplitudes that later on will be unitarized. Thus for instance in Refs. [61 64], the universal vector-meson coupling hypothesis is exploited to break the SU(4) symmetry in a convenient and well-defined manner. This is done by a $t$-channel exchange of vector mesons between pseudoscalar mesons and baryons, in such a way that chiral symmetry is preserved in the light meson sector, while the interaction is still of the Weinberg-Tomozawa (WT) type [67, 68]. A serious limitation of this approach is that it is not consistent with heavy-quark-spin symmetry, which is a proper QCD symmetry that appears when the quark masses become much larger than the typical confinement scale, $\Lambda_{\mathrm{QCD}}$. The recent works of Refs. [65, 66] develop a scheme consistent with both chiral and heavy-quark-spin symmetry by starting from a larger SU(8) spinflavor symmetry group, and conveniently breaking the spin (in the light $u, d, s$ sector) and flavor symmetries. In both schemes, coupled-channel unitarity plays a major role.

Coupled-channel unitarity has been also the essential ingredient in other recent works, where the vector mesonvector meson [69, 70] and vector meson-octet and decuplet baryon interactions $[71$ 73] in the light sector have been studied. In these cases, the formalism of the hidden gauge interaction for vector mesons of [74, 75] is adopted, and it has led to very successful results from a phenomenological point of view [76 78].

The approach taken in Refs. 41, 44], where $\mathrm{SU}(6)$ spin-flavor symmetry is invoked, allows to assign the vector mesons of the $\rho$ nonet and the pseudoscalar mesons of the $\pi$ octet in the same (35) SU(6) multiplet, while the baryons of the nucleon octet and $\Delta$ decuplet are placed in the totally symmetric $\mathbf{5 6} \mathrm{SU}(6)$ representation. The scheme is completely constrained by requiring that its restriction to the $\pi$-N octet sector will reduce to the $\mathrm{SU}(3) s$-wave WT Lagrangian, leading order (LO) of the chiral expansion in this case. Finally, the SU(6) symmetry is broken by using physical masses and decay constants. The corresponding BS amplitudes successfully reproduce the previous $\mathrm{SU}(3)$-flavor WT results for the lowest-lying $s$-and $d$-wave odd parity baryon resonances obtained from scattering of the mesons of the pion octet off baryons of the nucleon octet and delta decuplet [44]..$^{2}$ The extension of the scheme to the charm sector [65, 66] naturally accommodates heavy-quark-spin symmetry, as mentioned above, since it encodes spin symmetry in the charm sector, while this is not the case for those models based on vector meson exchanges.

In this work, we present the extension of the meson-baryon scheme derived in Ref. [41] to the meson-meson sector. The basis of our approach is rooted in the ideas of Caldi and Pagels [79, 80]. These authors identify vector mesons of the nonet as "dormant" Nambu-Goldstone bosons of an extended chiral symmetry $\mathrm{SU}(6)_{\mathrm{L}} \otimes \mathrm{SU}(6)_{\mathrm{R}}$. This symmetry is intrinsically an approximate one and the vector mesons acquire mass through relativistic corrections. Such scheme naturally solves a number of puzzles involving the nature and classification of vectors mesons and makes predictions in remarkable agreement with the experiment [79, 80]. The low energy theorems derived from partial conservation of the tensor current have been obtained in [81]. The Skyrmion of the $\mathrm{SU}(6)_{\mathrm{L}} \otimes \mathrm{SU}(6)_{\mathrm{R}}$ has been studied in [82]. The validity of the dormant Nambu-Goldstone boson description has been verified in the lattice [83].

Even if, for illustration purposes, $\mathrm{SU}(3)$ flavor symmetry is assumed to be exact and the pseudoscalar mesons are assumed to be massless (these simplifying assumptions are not essential and can be lifted) the breaking of $\mathrm{SU}(6)$ down to $\mathrm{SU}(3)$ (due to the vector mesons masses being nonvanishing) implies a breaking of $\mathrm{SU}(6)_{\mathrm{L}} \otimes \mathrm{SU}(6)_{\mathrm{R}}$ down to $\mathrm{SU}(3)_{\mathrm{L}} \otimes \mathrm{SU}(3)_{\mathrm{R}}$. One of the contributions of the present work is the construction of suitable Lagrangian mass terms achieving such pattern of symmetry breaking. Therefore the approximate spin-flavor symmetry scheme provides a unified framework to deal with lowest lying mesons, implementing the required symmetry breaking patterns and in particular fulfilling low energy theorems derived from chiral symmetry. In addition, when the present scheme is extended to include heavier quark flavors, the QCD heavy-quark spin symmetry can also be naturally accommodated in the spin-flavor approach through a suitable flavor breaking pattern. Although the predictions so obtained are not so reliable as those derived for pseudoscalar mesons assuming only the standard chiral symmetry breaking pattern of $\mathrm{QCD}$, the fact is that vector mesons do exist and they are known to play a relevant role in hadronic physics. Inescapably, they will interact among themselves and with other pseudoscalar mesons and will certainly influence the properties of the known mesons resonances. However not so many approaches to deal with vector mesons are available, and the existing ones, e.g., the hidden gauge approach [74, 75] are also subject to a certain amount of modeling not directly rooted in QCD. The theoretically founded models to deal with vector mesons being scarce, we regard the spin-flavor symmetric scenario (suitably broken) as a reasonable alternative approach and we believe it makes sense to work out the predictions of such a model. Moreover, we remarkably find that most of the low-lying even parity

\footnotetext{
2 The predictions of this scheme for the vector meson nonet-baryon decuplet sector have not been derived yet.
} 
PDG meson resonances can be classified according to multiplets of the spin-flavor symmetry group SU(6).

We will study the $s$-wave interaction of two members of the $\mathbf{3 5}$ ( $\pi$-octet $+\rho$-nonet) SU(6) multiplet by means of an enlarged WT meson Lagrangian to accommodate vector mesons, which guarantees that chiral symmetry is recovered when interactions between pseudoscalar Nambu-Goldstone bosons are being examined. We will pay a special attention to the novel pseudoscalar-vector $(P V)$ and vector-vector $(V V)$ channels, where we will compare our predictions with previous recent results [43, 69, 70] obtained within the formalism of the hidden gauge interaction for vector mesons. In the $P V \rightarrow P V$ sector, chiral symmetry constrains the interactions, and our model and that developed in Ref. [43] totally agree at LO in the chiral expansion, despite their different apparent structure and origin. As a result of this work, we show that most of the low-lying even parity meson resonances, specially in the $J^{P}=0^{+}$and $1^{+}$sectors, can be classified according to multiplets of the spin-flavor symmetry group $\mathrm{SU}(6)$. This can be seen in Table I], which summarizes the set of dynamically generated resonances obtained in this work. The remaining firmly established positive parity PDG meson states below $2 \mathrm{GeV}$, that cannot be accommodated within $\mathrm{SU}(6)$ multiplets, are clear candidates to be glueballs or hybrids. This is the case of the $f_{0}(1500), f_{1}(1420)$ and some $0^{+}\left(2^{++}\right)$resonances. On the other hand, we predict the existence of five exotic resonances $(I \geq 3 / 2$ and/or $|Y|=2)$ with masses in the region $1.4-1.6 \mathrm{GeV}$, which would complete the $27_{1}$ and $10_{3}$ and $10_{3}^{*} \mathrm{SU}(3) \otimes \mathrm{SU}(2)$ multiplets.

The extension of the model presented here to the charm sector would naturally accommodate heavy-quark spin symmetry. On the contrary, this latter QCD requirement will not be easily met for models [57 60] based on the formalism of the hidden gauge interaction for vector mesons, since those would not treat in the same way pseudoscalar $(D)$ and vector $\left(D^{*}\right)$ charmed mesons.

This paper is organized as follows: In Sect. III, we start from the chiral Lagrangian for pseudoscalar-pseudoscalar interactions (Subsect. IIA) and extend it to calculate the interaction vertices between two pseudoscalars, one pseudoscalar and one vector, and between two vector mesons in terms of $\mathrm{SU}(6)$ projectors and the corresponding eigenvalues (Subsect IB ). Also in this subsection, we show how to obtain three relations connecting these eigenvalues by matching our amplitudes to the LO ChPT ones for two pseudoscalar scattering and how finally all eigenvalues can be fixed. Next in Subsect. IID, we discuss the nature of the SU(6) symmetry breaking terms needed to account for the physical meson decay constant and masses, without spoiling partial conservation of the axial current in the light pseudoscalar sector. Sec [II deals with the BS equation and with issues related with its renormalization. In Sect. IV] we present results in terms of the unitarized amplitudes and search for poles on the complex plane. We discuss the results sector by sector trying to identify the obtained resonances or bound states with their experimental counterparts [84], and compare our results with earlier studies. A brief summary and some conclusions follow in Sect. V. In Appendix A the various potential matrices are compiled for the different hypercharge, isospin and spin sectors. In Appendix B details are given on the computation of the eigenvalues of various operators.

\section{SU(6) DESCRIPTION OF VECTOR-PSEUDOSCALAR AND VECTOR-VECTOR INTERACTIONS}

\section{A. SU(3) and chiral symmetry}

The lowest-order chiral Lagrangian describing the interaction of pseudoscalar Nambu-Goldstone bosons is [3]

$$
\mathcal{L}=\frac{f^{2}}{4} \operatorname{Tr}\left(\partial_{\mu} U^{\dagger} \partial^{\mu} U+\mathcal{M}\left(U+U^{\dagger}-2\right)\right)
$$

where $f \sim 90 \mathrm{MeV}$ is the chiral-limit pion decay constant, $U=e^{\mathrm{i} \sqrt{2} \Phi / f}$ is the $\mathrm{SU}(3)$ representation of the meson fields, with

$$
\Phi=\left(\begin{array}{ccc}
\frac{1}{\sqrt{6}} \eta+\frac{1}{\sqrt{2}} \pi^{0} & \pi^{+} & K^{+} \\
\pi^{-} & \frac{1}{\sqrt{6}} \eta-\frac{1}{\sqrt{2}} \pi^{0} & K^{0} \\
K^{-} & \bar{K}^{0} & -\sqrt{\frac{2}{3}} \eta
\end{array}\right),
$$

and the matrix $\mathcal{M}=\operatorname{diag}\left(m_{\pi}^{2}, m_{\pi}^{2}, 2 m_{K}^{2}-m_{\pi}^{2}\right)$ is determined by the pion and kaon meson masses. Expanding up to four meson fields, one finds

$$
\mathcal{L}^{\text {int }}=\frac{1}{12 f^{2}} \operatorname{Tr}\left(\left[\Phi, \partial_{\mu} \Phi\right]\left[\Phi, \partial^{\mu} \Phi\right]+\mathcal{M} \Phi^{4}\right)
$$


TABLE I: Tentative SU(6) classification of the poles found in this work, together with their possible PDG counterparts 84 . $J^{P}, Y, I^{G}$ stand for the spin-parity, hypercharge, isospin and $G$-parity, respectively [for non-strange states, charge conjugation is given by $\left.G=(-1)^{I}\right]$. Those resonances marked with $\dagger$ need to be confirmed, while a $(*)$ symbol indicates that the resonance does not appear in the PDG. Finally, a question mark symbol expresses our reservations on the assignment. Mixings between states with the same $J^{P} I^{G} Y$ quantum numbers, but belonging to different $\mathrm{SU}(6)$ and/or SU(3) multiplets have not been considered.

\begin{tabular}{|c|c|c|c|c|c|}
\hline \multicolumn{6}{|c|}{$J^{P}=0^{+}$} \\
\hline $\begin{array}{l}\mathrm{SU}(6) \\
\text { Irrep } \\
\end{array}$ & $\begin{array}{c}\mathrm{SU}(3) \otimes \mathrm{SU}(2) \\
\text { Irrep }\end{array}$ & $Y$ & $I^{G}$ & $\begin{array}{l}\sqrt{s}[\mathrm{MeV}] \\
\text { (this work) }\end{array}$ & PDG [84] \\
\hline 1 & $1_{1}$ & 0 & $0^{+}$ & $(635,-202)$ & $f_{0}(600)$ \\
\hline \multirow{3}{*}{$35_{s}$} & \multirow{3}{*}{81} & \pm 1 & $1 / 2$ & $(830,-170)$ & $K_{0}^{*}(800)^{\dagger}$ \\
\hline & & 0 & $1^{-}$ & $(991,-46)$ & $a_{0}(980)$ \\
\hline & & 0 & $0^{+}$ & $(969,0)$ & $f_{0}(980)$ \\
\hline \multirow{10}{*}{189} & \multirow{6}{*}{$27_{1}$} & \pm 2 & 1 & $(1564,-66)$ & $(*)$ \\
\hline & & \pm 1 & $3 / 2$ & $(1433,-70)$ & $(*)$ \\
\hline & & \pm 1 & $1 / 2$ & $(1428,-24)$ & $K_{0}^{*}(1430)$ \\
\hline & & 0 & $0^{+}$ & $(1350,-62)$ & $f_{0}(1370)$ \\
\hline & & 0 & $1^{-}$ & $(1442,-5)$ & $a_{0}(1450)$ \\
\hline & & 0 & $2^{+}$ & $(1419,-54)$ & $X(1420)^{\dagger}$ \\
\hline & \multirow{3}{*}{81} & \pm 1 & $1 / 2$ & $(1787,-37)$ & $K_{0}^{*}(1950)^{\dagger}$ \\
\hline & & 0 & $1^{-}$ & $(1760,-12)$ & $a_{0}(2020)^{\dagger} ?$ \\
\hline & & 0 & $0^{+}$ & $(1723,-52)$ & $f_{0}(1710)$ \\
\hline & $1_{1}$ & 0 & $0^{+}$ & - & \\
\hline
\end{tabular}

\begin{tabular}{|c|c|c|c|c|c|}
\hline \multicolumn{6}{|c|}{$J^{P}=1^{+}$} \\
\hline 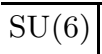 & $\mathrm{SU}(3) \otimes \mathrm{SU}(2)$ & $Y$ & $I^{G}$ & $\sqrt{s}[\mathrm{MeV}]$ & $\mathrm{PDG}[84]$ \\
\hline Irrep & Irrep & & & (this work) & \\
\hline \multirow{4}{*}{$35_{s}$} & \multirow{3}{*}{83} & \pm 1 & $1 / 2$ & $(1188,-64)$ & $K_{1}(1270)$ \\
\hline & & 0 & $1^{+}$ & $(1234,-57)$ & $b_{1}(1235)$ \\
\hline & & 0 & $0^{-}$ & $(1373,-17)$ & $h_{1}(1380)^{\dagger}$ \\
\hline & $1_{3}$ & 0 & $0^{-}$ & $(1006,-85)$ & $h_{1}(1170)$ \\
\hline \multirow{11}{*}{189} & \multirow{5}{*}{$\begin{array}{c}10_{3} \\
\& \\
10_{3}^{*}\end{array}$} & \pm 2 & 0 & $(1608,-114)$ & $(*)$ \\
\hline & & \pm 1 & $3 / 2$ & $(1499,-127)$ & $(*)$ \\
\hline & & \pm 1 & $1 / 2$ & $(1414,-66)$ & $K_{1}(1400) ?$ \\
\hline & & 0 & $1^{+}$ & $(1642,-139)$ & $b_{1}(1960)^{\dagger} ?$ \\
\hline & & 0 & $1^{-}$ & $(1568,-145)$ & $a_{1}(1640)^{\dagger}$ \\
\hline & \multirow{3}{*}{$8_{3}^{a}$} & \pm 1 & $1 / 2$ & $(1250,-31)$ & $K_{1}(\ldots)(*)$ \\
\hline & & 0 & $1^{-}$ & $(1021,-251)$ & $a_{1}(1260)$ \\
\hline & & 0 & $0^{+}$ & $(1286,0)$ & $f_{1}(1285)$ \\
\hline & \multirow{3}{*}{$8_{3}^{S}$} & \pm 1 & $1 / 2$ & $(1665,-95)$ & $K_{1}(1650)^{\dagger} ?$ \\
\hline & & 0 & $1^{+}$ & - & \\
\hline & & 0 & $0^{-}$ & $(1600,-67)$ & $h_{1}(1595)^{\dagger}$ \\
\hline
\end{tabular}

\begin{tabular}{|c|c|c|c|c|c|}
\hline \multicolumn{6}{|c|}{$J^{P}=2^{+}$} \\
\hline $\begin{array}{l}\mathrm{SU}(6) \\
\text { Irrep }\end{array}$ & $\begin{array}{c}\mathrm{SU}(3) \otimes \mathrm{SU}(2) \\
\text { Irrep }\end{array}$ & $Y$ & \begin{tabular}{|l|}
$G$ \\
\end{tabular} & $\begin{array}{l}\sqrt{s}[\mathrm{MeV}] \\
\text { (this work) }\end{array}$ & PDG [84] \\
\hline 189 & \multirow{3}{*}{85} & \pm 1 & $1 / 2$ & $(1708,-156)$ & $K_{2}^{*}(1430) ?$ \\
\hline \multirow{3}{*}{$\begin{array}{c}\& \\
\text { Contact } V V\end{array}$} & & 0 & $1^{-}$ & $(1775,-6)$ & $a_{2}(1700)^{\dagger}$ \\
\hline & & 0 & $0^{+}$ & $(1783,-19)$ & $f_{2}(1640)^{\dagger} ?$ \\
\hline & $1_{5}$ & 0 & $0^{+}$ & $(1289,0)$ & $f_{2}(1270)$ \\
\hline \multirow{4}{*}{ Contact $V V$} & \multirow{3}{*}{85} & \pm 1 & $1 / 2$ & \multirow{3}{*}{$\begin{array}{c}- \\
(1228,0) \\
-\end{array}$} & \multirow{3}{*}{$a_{2}(1320)$} \\
\hline & & 0 & $1^{-}$ & & \\
\hline & & 0 & $0^{+}$ & & \\
\hline & $1_{5}$ & 0 & $0^{+}$ & - & \\
\hline
\end{tabular}

Taking a common mass, $m$, for all the (pseudo) Nambu-Goldstone mesons and projecting into $s$-wave, the above Lagrangian leads to an interaction Hamiltonian (on shell)

$$
\mathcal{H}=\frac{3 s-4 m^{2}}{6 f^{2}} \hat{H}_{1}-\frac{m^{2}}{3 f^{2}} \hat{H}_{2}
$$

where $\sqrt{s}$ is the total energy of the meson pair in the center of mass system, and $\hat{H}_{1}$ and $\hat{H}_{2}$ are coupled-channel matrices; they are $I Y$ block diagonal, with $I$ and $Y$ the total isospin and hypercharge (strangeness) of either the initial or final meson pair. The normalization can be unambiguously fixed thanks to the relation of the diagonal matrix elements of $\mathcal{H}(s)$ with the $s$-wave scattering amplitude, $\mathcal{F}(s)$, the phase shifts $\delta(s)$ and inelasticities $\eta(s)$,

$$
\mathcal{H}_{i i}(s)=-8 \pi \sqrt{s} \mathcal{F}_{i i}(s), \quad 2 \mathrm{i} p \mathcal{F}=\eta e^{2 \mathrm{i} \delta}-1,
$$

where $p$ is the momentum in the center of mass frame of the two mesons.

The operators $\hat{H}_{1}$ and $\hat{H}_{2}$ are linear combinations of orthogonal projectors, $P_{\mu}$, onto the $\mathrm{SU}(3) \mu$ representations 
that appear in the reduction of the product of representations $8 \otimes 8$. Namely,

$$
\hat{H}_{1}=-3 P_{1}-\frac{3}{2} P_{8_{s}}+P_{27}, \quad \hat{H}_{2}=5 P_{1}+P_{8_{s}}+P_{27} .
$$

Note that only representations which are symmetric under the permutation of the two octets appear. This is a consequence of $s$-wave Bose statistics, once we have assigned a common mass for all pseudoscalar mesons.

On the other hand, by imposing just SU(3) flavor symmetry, the interaction Hamiltonian would be of the form

$$
\mathcal{H}(s)=\sum_{\mu} F_{\mu}(s) P_{\mu}
$$

with the $\mathrm{SU}(3)$ representation $\mu$ running over the $1,8_{s}$ and 27 irreducible symmetric representations that appear in the reduction of $8 \otimes 8$, and $F_{\mu}$ arbitrary functions of the Mandelstam variable $s$. The approximate chiral symmetry of QCD, which is much more restrictive than just flavor symmetry, and its pattern of spontaneous and explicit symmetry breaking fixes this enormous freedom and allows to determine the chiral expansion of the functions $F_{\mu}(s)$. At LO, the functions $F_{\mu}$ can be easily read off from Eqs. (4) and (6).

The first contribution in $\mathcal{L}^{\text {int }}$ of Eq. (3) is the WT term in this $\pi \pi$ case. There is a WT term for the interaction of Nambu-Goldstone bosons off any target. Its form follows entirely from chiral symmetry (and its pattern of symmetry breaking) [67, 68] and fully accounts for the interaction near threshold. Specifically, assuming exact chiral symmetry (and so massless Nambu-Goldstone bosons) and for $s$-wave, $\mathcal{H}(s)$ vanishes at threshold and moreover

$$
\left.\frac{d \mathcal{H}(s)}{d s}\right|_{\text {threshold }}=\xi \frac{1}{2 f^{2}} \hat{H}_{\mathrm{WT}}
$$

where $\xi$ is the symmetry factor, namely, $1 / 2$ if the target is another Nambu-Goldstone boson and 1 if it is not, and

$$
\hat{H}_{\mathrm{WT}}=\sum_{\mu} \lambda_{\mu} P_{\mu}
$$

where $\mu$ runs over the allowed $\mathrm{SU}(3)$ representations. Note that $\mathcal{H}(s)$ acts on different spaces depending on the target, e.g., $(8 \otimes 8)_{\text {sym }}$ for $\pi \pi, 8 \otimes 8$ for $\pi \rho$, and $8 \otimes 1$ for $\pi \omega_{1}$. For two flavors the WT interaction comes as the scalar product of the Nambu-Goldstone boson and target isospin operators [85] (and so it depends only on the isospin target). For any number $N_{F}$ of (massless) flavors one has instead $\sum_{a=1}^{N_{F}^{2}-1} J_{\mathrm{NG}}^{a} J_{\text {target }}^{a}$ and this fixes the eigenvalues (see e.g. [86])

$$
\lambda_{\mu}=C_{2}(\mu)-C_{2}\left(\mu_{\mathrm{NG}}\right)-C_{2}\left(\mu_{\text {target }}\right),
$$

where $C_{2}(\mu)$ refers to the value of the quadratic Casimir of the irrep $\mu$ in $\mathrm{SU}\left(N_{F}\right)$ (with normalization $\left.C_{2}(\operatorname{adj})=N_{F}\right)$, $\mu_{\mathrm{NG}}$ is the adjoint representation.

This gives for $\pi \pi$ scattering the eigenvalues quoted in (6) for $N_{F}=3$, and new ones for $\pi \rho$ and $\pi \omega_{1}\left(\omega_{1}\right.$ refers to the $\mathrm{SU}(3)$ singlet):

$$
\lambda_{1}^{\pi}=\lambda_{1}^{\rho}=-6, \quad \lambda_{8_{s}}^{\pi}=\lambda_{8_{s}}^{\rho}=\lambda_{8_{a}}^{\rho}=-3, \quad \lambda_{27}^{\pi}=\lambda_{27}^{\rho}=2, \quad \lambda_{10}^{\rho}=\lambda_{10^{*}}^{\rho}=0, \quad \lambda_{8}^{\omega_{1}}=0 .
$$

Exact SU(3) symmetry has been assumed throughout in this discussion, so $\pi$ refers to the full $\pi$ octet, and so on. Note that no configuration mixing (e.g. $\left.\left|\pi \rho ; 8_{s}\right\rangle \rightarrow\left|\pi \rho ; 8_{a}\right\rangle\right)$ takes place within the WT interaction. These results will be used next.

\section{B. Spin-flavor and chiral symmetries}

With the inclusion of spin there are 36 quark-antiquark $(q \bar{q})$ states, and the $\mathrm{SU}(6)$ group representation reduction (denoting the $\mathrm{SU}(6)$ multiplets by their dimensionality and an $\mathrm{SU}(3)$ multiplet $\mu$ of spin $J$ by $\mu_{2 J+1}$ ) reads

$$
\mathbf{6} \otimes \mathbf{6}^{*}=\mathbf{3 5} \oplus \mathbf{1}=\underbrace{8_{1} \oplus 8_{3} \oplus 1_{3}}_{\mathbf{3 5}} \oplus \underbrace{1_{1}}_{\mathbf{1}} .
$$

The lowest bound $q \bar{q}$ state is expected to be an $s$-state and the relative parity of a fermion-antifermion pair being odd, the octet of pseudoscalar $(K, \pi, \eta, \bar{K})$ and the nonet of vector $\left(K^{*}, \rho, \omega, \bar{K}^{*}, \phi\right)$ mesons are commonly placed in the 35 representation 87 89]. 
Strong interaction conserves total spin $(J)$, hypercharge $(Y)$, and isospin $(I)$ (assuming equal masses for the up and down quarks). Furthermore, since we consider only $s$-wave states, the total spin of the meson-meson states is simply the sum of their individual spins. Therefore, on account of the SU(6) group reduction

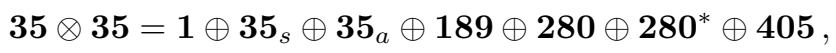

a meson-meson state written in terms of the $\mathrm{SU}(6)$ basis takes the form

$$
\left|M_{1} M_{2} ; Y I J\right\rangle=\sum_{\mu, \alpha, \mathcal{R}}\left(\begin{array}{cc|c}
\mu_{M_{1}} & \mu_{M_{2}} & \mu \\
I_{M_{1}} Y_{M_{1}} & I_{M_{2}} Y_{M_{2}} & I Y
\end{array}\right)\left(\begin{array}{cc|c}
35 & 35 & \mathcal{R} \\
\mu_{M_{1}} J_{M_{1}} & \mu_{M_{2}} J_{M_{2}} & \mu J \alpha
\end{array}\right)\left|\mathcal{R} ; \mu_{2 J+1}^{\alpha} I Y\right\rangle,
$$

where $Y=Y_{M_{1}}+Y_{M_{2}},\left|I_{M_{1}}-I_{M_{2}}\right| \leq I \leq I_{M_{1}}+I_{M_{2}},\left|J_{M_{1}}-J_{M_{2}}\right| \leq J \leq J_{M_{1}}+J_{M_{2}}, \mu$ and $\mathcal{R}$ denote generic SU(3) and SU(6) representations, respectively. $I_{M_{1,2}}, Y_{M_{1,2}}, J_{M_{1,2}}$ are the isospin, hypercharge, and spin of the two mesons. In the above equation, $\mathcal{R}=\mathbf{1}, \mathbf{1 8 9}, \mathbf{3 5}_{s}, \mathbf{4 0 5}, \mathbf{3 5}_{a}, \mathbf{2 8 0}$ and $\mathbf{2 8 0 ^ { * }}, \mu=1,8_{s}, 8_{a}, 27,10,10^{*}$, and $\alpha$ accounts for the multiplicity of each of the $\mu_{2 J+1} \mathrm{SU}(3)$ multiplets of spin $J$. In Eq. (14), the two coefficients multiplying each element of the $\mathrm{SU}(6)$ basis, $\left|\mathcal{R}, \mu_{2 J+1}^{\alpha} I Y\right\rangle$, are the $\mathrm{SU}(3)$ isoscalar factors 90] and the $\mathrm{SU}(6)$ Clebsch-Gordan coefficients [91], respectively.

At this point one can ask how does $\mathrm{SU}(6)$ symmetry go along with chiral symmetry. Certainly, because chiral symmetry must be present in any reliable approach this is a central point in this work. To clarify this issue we will consider the following exercise, namely, whether it is possible for an $\mathrm{SU}(6)$ invariant interaction to reproduce the low energy theorems quoted in Eq. (8) with the correct eigenvalues in Eq. (11). (Again we assume exact chiral symmetry and $s$-wave.) As it turns out, this is indeed possible. Such solutions correspond to operators $\mathcal{H}^{\mathrm{SU}(6)}(s)$ acting on the spin-flavor space $\mathbf{3 5} \otimes \mathbf{3 5}$ of the form

$$
\mathcal{H}^{\mathrm{SU}(6)}(s)=\frac{1}{2 f^{2}} \sum_{\mathcal{R}} F_{\mathcal{R}}(s) \lambda_{\mathcal{R}} P_{\mathcal{R}}
$$

where $\mathcal{R}$ runs over the seven $\mathrm{SU}(6)$ irreps in Eq. (13) and $P_{\mathcal{R}}$ are the corresponding projectors. The functions $F_{\mathcal{R}}(s)$ vanish at threshold and are normalized by the condition $d F_{\mathcal{R}}(s) /\left.d s\right|_{\text {threshold }}=1$, e.g. $F_{\mathcal{R}}(s)=s-\frac{1}{2} \sum_{i=1}^{4} q_{i}^{2}$. Therefore,

$$
\left.\frac{d \mathcal{H}^{\mathrm{SU}(6)}(s)}{d s}\right|_{\text {threshold }}=\frac{1}{2 f^{2}} \hat{H}_{\mathrm{WT}}^{\mathrm{SU}(6)}
$$

with

$$
\hat{H}_{\mathrm{WT}}^{\mathrm{SU}(6)}=\sum_{\mathcal{R}} \lambda_{\mathcal{R}} P_{\mathcal{R}}
$$

Finally, the eigenvalues $\lambda_{\mathcal{R}}$ reproducing those in (11) are $^{3}$

$$
\lambda_{\mathbf{1}}=-12, \quad \lambda_{\mathbf{3 5}}=\lambda_{\mathbf{3 5}_{a}}=-6, \quad \lambda_{\mathbf{1 8 9}}=-2, \quad \lambda_{\mathbf{4 0 5}}=2, \quad \lambda_{\mathbf{2 8 0}}=\lambda_{\mathbf{2 8 0}}=0 .
$$

Several comments are pertinent here. i) The functions $F_{\mathcal{R}}(s)$ depend on the concrete model. Chiral symmetry fixes the derivative of $\mathcal{H}^{\mathrm{SU}(6)}(s)$ with respect to $s$ at threshold. (A detailed model is developed below.) ii) The eigenvalues $\lambda_{\mathcal{R}}$ are unique and are such that $\hat{H}_{\mathrm{WT}}^{\mathrm{SU}(6)}$, when restricted to the $\pi \pi, \pi \rho$ and $\pi \omega_{1}$ subspaces yield the correct $\mathrm{SU}(3)$ eigenvalues of subsection $\amalg \mathrm{A}$. iii) The projectors on antisymmetric representations vanish on $P P$ states. However, for the more general case involving vector mesons, both symmetric and antisymmetric representations (e.g. $\mathbf{3 5}_{s}$ and $\mathbf{3 5}_{a}$ ) are required even in $s$-wave. Although the $\pi$ octet and the $\rho$ nonet fall in the same $\mathrm{SU}(6)$ representation they are kinematically distinguishable through their mass. To give mass to the vector mesons certainly requires breaking $\mathrm{SU}(6)$ in the Lagrangian (not only through mass terms but also by interaction terms, due to chiral symmetry). This

${ }^{3}$ For $\pi M$ scattering the relation between $\mathrm{SU}(3)$ and $\mathrm{SU}(6)$ eigenvalues is
\[ \lambda_{\mu_{2 J+1} \alpha}^{M}=2 \sum_{\mathcal{R}} \lambda_{\mathcal{R}}\left(\begin{array}{cc|c}\mathbf{3 5} & \mathbf{3 5} & \mathcal{R} \\ 8 & \mu_{2 J+1}^{M} & \mu_{2 J+1} \alpha\end{array}\right)^{2} . \] 
simply means that $\mathcal{H}^{\mathrm{SU}(6)}(s)$ is not the full $\mathcal{H}(s)$ acting on the space $\mathbf{3 5} \otimes \mathbf{3 5}$. Besides $\mathcal{H}^{\mathrm{SU}(6)}(s)$ there are further terms, $\delta \mathcal{H}(s)$, which do not have a contribution to $\mathcal{H}\left(s_{\text {threshold }}\right)$ nor $d \mathcal{H}(s) /\left.d s\right|_{\text {threshold }}$ when they are restricted to the subspaces $P P \rightarrow P P$ or $P V \rightarrow P V$. (Once again we refer to the model below which fulfills these requirements.) iv) $\hat{H}_{\mathrm{WT}}^{\mathrm{SU}(6)}$ can be regarded as an extension from $\mathrm{WT}$ in flavor $\mathrm{SU}(3)$ to a WT-like term in spin-flavor $\mathrm{SU}(6)$. The eigenvalues $\lambda_{\mathcal{R}}$ in Eq. (19) obey the general WT rule in Eq. (10) applied to SU(6) instead of SU(3). Actually there is an extra factor of two in Eq. (16) since the symmetry factor is $\xi=1 / 2$ for $\mathbf{3 5} \times \mathbf{3 5}$. However this is not related to the validity of Eq. (10) in the SU(6) extended version of WT but to the fact that $f_{6}=f / \sqrt{2}$ applies instead of $f$ in this extended version. (The same factor 2 appears in Eq. (18).) No such factors appear when $\mathrm{SU}\left(N_{F}\right)$ is extended to $\mathrm{SU}\left(N_{F}^{\prime}\right)$ (a larger number or flavors). This is because the embedding of $\mathrm{SU}\left(N_{F}\right)$ into $\mathrm{SU}\left(N_{F}^{\prime}\right)$ is different from the embedding of $\mathrm{SU}\left(N_{F}\right)$ into spin-flavor $\mathrm{SU}\left(2 N_{F}\right)$. (See below.) As will be obvious from what follows the same exercise can be repeated, successfully, for any number of flavors and not only for meson-meson scattering but also for meson-baryon [41, 49].

The previous discussion suggests that chiral symmetry, $\mathrm{SU}(3)_{\mathrm{L}} \otimes \mathrm{SU}(3)_{\mathrm{R}}$, is compatible with spin-flavor symmetry, $\mathrm{SU}(6)$. (Note that 10 couplings, $\lambda_{\mu}$, have been reproduced using only 7 unknowns, $\lambda_{\mathcal{R}}$, and a similar overdetermination exists for more flavors of for meson-baryon.) In fact such compatibility was exposed by Caldi and Pagels in [79, 80] by the simple method of extending $\mathrm{SU}(3)_{\mathrm{L}}$ to $\mathrm{SU}(6)_{\mathrm{L}}$ and $\mathrm{SU}(3)_{\mathrm{R}}$ to $\mathrm{SU}(6)_{\mathrm{R}}$ where $\mathrm{SU}(6)$ refers to spin-flavor. This produces a larger symmetry group, $\mathrm{SU}(6)_{\mathrm{L}} \otimes \mathrm{SU}(6)_{\mathrm{R}}$, which includes chiral and spin-flavor groups as subgroups. Specifically, the usual spin-flavor SU(6) corresponds to the subgroup of diagonal transformations (i.e., the same SU(6) transformation in $\mathrm{L}$ and $\mathrm{R}$ sectors) similar to $\mathrm{SU}(3)_{\mathrm{V}}$ (flavor group) in $\mathrm{SU}(3)_{\mathrm{L}} \otimes \mathrm{SU}(3)_{\mathrm{R}}$.

The spin-flavor extended chiral group $\mathrm{SU}(6)_{\mathrm{L}} \otimes \mathrm{SU}(6)_{\mathrm{R}}$ is a realization of the Feynman-Gell-Mann-Zweig algebra [92] and was introduced in [79, 80] precisely to solve an apparent inconsistency. Namely, on the one hand the phenomenological successful spin-flavor symmetry in the quark model puts $\pi$ and $\rho$ in the same SU(6) multiplet. On the other, the pion is a collective state, the Nambu-Goldstone boson from spontaneous breaking of chiral symmetry. In the scenario of [79, 80], one would find that in an exactly SU(6) symmetric world the $\pi$ octet and the $\rho$ nonet are the Nambu-Goldstone bosons of the spontaneous breaking of $\mathrm{SU}(6)_{\mathrm{L}} \otimes \mathrm{SU}(6)_{\mathrm{R}}$ down to $\mathrm{SU}(6)$. Spin-flavor symmetry is an approximated one and so the $\rho$ nonet mesons are regarded as "dormant" Nambu-Goldstone bosons. As it is known, spin-flavor symmetries cannot be exact as they cannot be accommodated with full Poincare invariance [93]. Still one can consider a static limit enjoying $\mathrm{SU}(6)_{\mathrm{L}} \otimes \mathrm{SU}(6)_{\mathrm{R}}$ symmetry. In the Caldi-Pagels scenario, relativistic (and so SU(6) breaking) corrections give mass to the vector mesons while pions are still protected by the usual SU(3) chiral symmetry.

The scenario just described solves a number of puzzles involving vector mesons while maintaining vector meson dominance, KSFR relations and so on [79, 80]. Here we comment only on two issues, namely, the consequences regarding the chiral and Lorentz transformations of vector mesons. Because the pion falls in the $\left(3,3^{*}\right)+\left(3^{*}, 3\right)$ representation of the chiral group, spin-flavor symmetry requires the $\rho$ to fall in the same representation (and both in $\left(6,6^{*}\right)+\left(6^{*}, 6\right)$ of $\left.\mathrm{SU}(6)_{\mathrm{L}} \otimes \mathrm{SU}(6)_{\mathrm{R}}\right)$. This is different from vector and axial currents which transform instead as $(8,1)+(1,8)$ under the chiral group. At first the fact that the $\rho$ meson and the vector current transform differently seems to be in conflict with vector meson dominance. As shown in [79, 80] this not so, due to the spontaneous breaking of chiral symmetry, for the same reason that PCAC relates pion and axial current, also in different chiral representations.

Related to the chiral representation is the nature of vectors mesons under Lorentz transformations. This is most easily exposed by coupling the meson fields to quark bilinears (alternatively the quark bilinear can be regarded as a representation or interpolating field of the meson, as in Nambu-Jona-Lasinio models). Let us for this discussion consider just two flavors $\left(N_{F}=2\right)$ and use a linear sigma model representation (as opposed to the non-linear one) as there it is simpler to expose the chiral transformation properties of the fields. The pion and $\sigma$ mesons couple to $\bar{q} i \gamma_{5} \vec{\tau} q$ and $\bar{q} q$. Of course, this just of the form $\bar{q}_{\mathrm{L}} \mathcal{M} q_{\mathrm{R}}+\bar{q}_{\mathrm{R}} \mathcal{M}^{\dagger} q_{\mathrm{L}}$ corresponding to the chiral representation $(1 / 2,1 / 2)$. The coupling can be extended to include vector mesons while preserving spin-flavor

$$
\mathcal{M}=\sigma+i \pi_{a} \tau_{a}+i \rho_{a i} \tau_{a} \sigma_{i}+\cdots .
$$

(Let us remark that these are the linear sigma model mesons fields and will be used only in this subsection. Elsewhere in this paper the non-linear meson fields are used. Also note that this $\mathcal{M}$ is unrelated to the mass term in Eq. (1).) The dots represent further meson fields to complete a general $2 N_{F} \times 2 N_{F}$ complex matrix. The space spanned by such matrices $\mathcal{M}$ carries a representation of the group $\mathrm{SU}\left(2 N_{F}\right)_{\mathrm{L}} \otimes \mathrm{SU}\left(2 N_{F}\right)_{\mathrm{R}}$, acting as $\mathcal{M} \rightarrow \Omega_{\mathrm{L}}^{\dagger} \mathcal{M} \Omega_{\mathrm{R}}$ with $\Omega_{\mathrm{L}, \mathrm{R}} \in \mathrm{SU}\left(2 N_{F}\right)$. This $\mathrm{SU}\left(2 N_{F}\right) \supset \mathrm{SU}\left(N_{F}\right) \otimes \mathrm{SU}(2)$ is generated by $\tau_{a}, \sigma_{i}$ and $\tau_{a} \sigma_{i}$. In particular, the $\sigma$ and $\pi$ fields mix under transformations generated by $\tau_{a}$ with $\Omega_{\mathrm{L}} \neq \Omega_{\mathrm{R}}$. These are the usual chiral transformations. On the other hand $\pi$ mixes with $\rho$ (and other mesons) under spin-flavor transformations $\left(\Omega_{\mathrm{L}}=\Omega_{\mathrm{R}}\right)$. Using the chiral representation of the Dirac gammas one immediately obtains the coupling

$$
\bar{q}_{\mathrm{L}} \mathcal{M} q_{\mathrm{R}}+\bar{q}_{\mathrm{R}} \mathcal{M}^{\dagger} q_{\mathrm{L}}=\bar{q}\left(\sigma+i \pi_{a} \tau_{a} \gamma_{5}+\rho_{a i} \tau_{a} \sigma^{0 i}+\cdots\right) q .
$$


The LR+RL structure (i.e. $\left.\left(3,3^{*}\right)+\left(3^{*}, 3\right)\right)$ requires quark bilinears constructed with $1, \gamma_{5}$ or $\sigma_{\mu \nu}$ which commute with $\gamma_{5}$, while $\gamma_{\mu}$ or $\gamma_{\mu} \gamma_{5}$ produce LL+RR (i.e. $(1,8)+(8,1)$ ). This implies that the spin-flavor approach favors an antisymmetric tensor representation of vector mesons [2]. The antisymmetric tensor $V_{\mu \nu}$ contains 3 spurious degrees of freedom and one can choose to take $V_{0 i}$ as the dynamical fields [94]. This is the choice in Eq. (21) with $\rho_{a i} \sim \bar{q} \tau_{a} \sigma^{0 i} q$. The description of vector mesons using antisymmetric tensors has been shown to be consistent with all expected properties of vector mesons [85, 94, 95]. (See e.g. [96] for the use of $\bar{q} \sigma_{\mu \nu} q$ as interpolating field of the vector meson in the context of Nambu-Jona-Lasinio models.)

Conversely, the quark bilinear construction naturally favors a $\left(3,3^{*}\right)+\left(3^{*}, 3\right)$ representation instead of $(1,8)+(8,1)$ for vector mesons if they are considered as antisymmetric tensors. In principle, one would expect that the chiral representation under which the meson transforms would reflect itself on the observable properties of the meson. However, this is not at all obvious. In the context of effective chiral Lagrangians for mesons a very convenient treatment is that based on the non-linear realization of chiral symmetry [97]. (Let us remark that only the linear realization is used in this work.) In this approach a field $u$ is constructed out of $\mathcal{M}$ such that under chiral transformations $u \rightarrow \Omega_{\mathrm{L}}^{\dagger} u h=h^{\dagger} u \Omega_{\mathrm{R}}$ (where $u$ and $h$ are unitary matrices) [85]. A field of the type LL such as the chiral current $V_{\mathrm{L}}^{\mu}$ transforms as $\Omega_{\mathrm{L}}^{\dagger} V_{\mathrm{L}}^{\mu} \Omega_{\mathrm{L}}$, and so belongs to the chiral representation $(8,1)$. This field is represented in the non-linear realization by the new field $\tilde{V}_{\mathrm{L}}^{\mu}=u^{\dagger} V_{\mathrm{L}}^{\mu} u$ which transforms instead as $h^{\dagger} \tilde{V}_{\mathrm{L}}^{\mu} h$. Likewise, the field $\mathcal{M}$, of the type LR, will be represented by $\tilde{\mathcal{M}}=u^{\dagger} \mathcal{M} u^{\dagger}$. This new field transforms as $h^{\dagger} \tilde{\mathcal{M}} h$, that is, exactly in the same way as the chiral currents or the vector or axial currents, etc. That was precisely the point of the non-linear realization, namely, all fields in the same representation with respect to $\mathrm{SU}\left(N_{F}\right)_{V}$ will be represented by fields transforming in the same way under general chiral transformations, regardless of their detailed chiral representation. Therefore, such detailed chiral representation does not reflect on the properties of the meson, at least to the extend that effective chiral Lagrangians are sufficient to describe them. This should not be surprising as it was already noted before that, e.g., the WT term is only sensible to the isospin (or more generally, flavor) of the target.

\section{The model: SU(6) invariant part}

In view of the previous remarks, we introduce now a model for meson interaction, including the $\pi$ octet and the $\rho$ nonet, with simultaneous chiral symmetry and spin-flavor symmetry, suitably broken.

The natural $\mathrm{SU}(6)$ extension of Eq. (1) from $\mathrm{SU}(3)$ to $\mathrm{SU}(6)$ is

$$
\mathcal{L}_{\mathrm{SU}(6)}=\frac{f_{6}^{2}}{4} \operatorname{Tr}\left(\partial_{\mu} U_{6}^{\dagger} \partial^{\mu} U_{6}+\mathcal{M}_{6}\left(U_{6}+U_{6}^{\dagger}-2\right)\right), \quad U_{6}=e^{\mathrm{i} \sqrt{2} \Phi_{6} / f_{6}} .
$$

$U_{6}$ is a unitary $6 \times 6$ matrix that transforms under the linear realization of $\mathrm{SU}(6)_{\mathrm{L}} \otimes \mathrm{SU}(6)_{\mathrm{R}}$. The Hermitian matrix $\Phi_{6}$ is the meson field, in the $\mathbf{3 5}$ irreducible representation of $\mathrm{SU}(6)$, and $f_{6}=f / \sqrt{2}$, as shown in Appendix B of Ref. [49]. The first term in $\mathcal{L}_{\mathrm{SU}(6)}$ preserves both chiral and spin-flavor symmetry. The second term breaks chiral symmetry and possibly flavor symmetry. This is not the most general breaking and this issue will be discussed in the next subsection. For the time being this term will be kept for illustration purposes with $\mathcal{M}_{6}=m_{6} I_{6 \times 6}$, with $m_{6}$ a common mass for all mesons belonging to the $\mathrm{SU}(6) \mathbf{3 5}$ irreducible representation.

Expanding the previous Lagrangian up to $\mathcal{O}\left(\Phi_{6}^{4}\right)$ gives the interaction Lagrangian,

$$
\mathcal{L}_{\mathrm{SU}(6)}^{\mathrm{int}}=\frac{1}{12 f_{6}^{2}} \operatorname{Tr}\left(\left[\Phi_{6}, \partial_{\mu} \Phi_{6}\right]\left[\Phi_{6}, \partial^{\mu} \Phi_{6}\right]+m_{6} \Phi_{6}^{4}\right) .
$$

The restriction of this Lagrangian to the $\mathrm{SU}(3)$ pseudoscalar $8 \otimes 8$ sector reproduces that given in Eq. (3) (with a common mass for all pseudoscalars). The kinetic term in Eq. (23) amounts to a coupling of the type $\left[(\mathbf{3 5} \otimes 35)_{35_{a}} \otimes(35 \otimes \mathbf{3 5})_{35_{a}}\right]_{\mathbf{1}}$ in the $t$-channel. That is, each meson pair, respecting Bose statistics, is coupled to the antisymmetric $\mathrm{SU}(6)$ adjoint representation $\left(\mathbf{3 5}_{a}\right)$ and the two resulting $\mathbf{3 5} \mathbf{5}_{a}$ 's couple into the singlet one to built up an SU(6) invariant interaction. This mechanism is completely analogous to that in Eq. (3) for SU(3) and so it is a natural extension of the WT chiral Lagrangian.

$\Phi_{6}$ is a dimension six matrix made of full meson fields, which depend on the space-time coordinates. A suitable 
choice for the $\Phi_{6}$ field is ${ }^{4}$

$$
\Phi_{6}=\underbrace{P_{a} \frac{\lambda_{a}}{\sqrt{2}} \otimes \frac{I_{2 \times 2}}{\sqrt{2}}}_{\Phi_{P}}+\underbrace{R_{a k} \frac{\lambda_{a}}{\sqrt{2}} \otimes \frac{\sigma_{k}}{\sqrt{2}}+W_{k} \frac{\lambda_{0}}{\sqrt{2}} \otimes \frac{\sigma_{k}}{\sqrt{2}}}_{\Phi_{V}}, \quad a=1, \ldots, 8, \quad k=1,2,3
$$

with $\lambda_{a}$ the Gell-Mann and $\vec{\sigma}$ the Pauli spin matrices, respectively, and $\lambda_{0}=\sqrt{2 / 3} I_{3 \times 3}\left(I_{n \times n}\right.$ denotes the identity matrix in the $n$ dimensional space). $P_{a}$ are the $\pi, K, \eta$ fields, while $R_{a k}$ and $W_{k}$ stand for the $\rho$-vector nonet fields, considering explicitly the spin degrees of freedom. The annihilation part of the meson matrix $\left[\Phi_{6}\right]_{j}^{i}$ is determined by the operators $M^{i}{ }_{j}$. Regarding $M$ as a matrix with respect to $i$ and $j$, the convention is that the upper/lower index acts as the first/second index of the matrix. $M$ is traceless and transforms under $\mathrm{SU}(6)$ in the same way as the quark operators

$$
q^{i} \bar{q}_{j}-\frac{1}{2 N_{F}} q^{m} \bar{q}_{m} \delta_{j}^{i}, \quad i, j=1, \ldots 2 N_{F},
$$

where $N_{F}$ is the number of flavors, three in this work.

We have denoted the contravariant and covariant spin-flavor quark and antiquark components

$$
q^{i}=\left(\begin{array}{c}
u \uparrow \\
d \uparrow \\
s \uparrow \\
u \downarrow \\
d \downarrow \\
s \downarrow
\end{array}\right), \quad \bar{q}_{i}=(\bar{u} \downarrow,-\bar{d} \downarrow,-\bar{s} \downarrow,-\bar{u} \uparrow, \bar{d} \uparrow, \bar{s} \uparrow)
$$

where $q^{i}\left(\bar{q}_{i}\right)$ annihilates ${ }^{5}$ a quark (antiquark) with the spin-flavor $i$. For instance $\bar{u} \downarrow$ annihilates an antiquark with flavor $\bar{u}$ and $S_{z}=-1 / 2$. The corresponding Wick's contractions of these operators read

$$
M_{l}^{k} M_{j}^{\dagger i}=\delta_{j}^{k} \delta_{l}^{i}-\frac{1}{2 N_{F}} \delta_{j}^{i} \delta_{l}^{k}
$$

For the process depicted in Fig. 1, the Lagrangian of Eq. (23) provides the following amplitudes $\left(\mathcal{H}^{\mathrm{SU}(6)}\right)$

$$
\mathcal{H}^{\mathrm{SU}(6)}=\mathcal{H}_{+}^{\mathrm{SU}(6)}+\mathcal{H}_{-}^{\mathrm{SU}(6)}
$$

with

$$
\begin{aligned}
\mathcal{H}_{+}^{\mathrm{SU}(6)} & =\frac{1}{12 f_{6}^{2}}\left(3 s-\sum_{i=1}^{4} q_{i}^{2}\right)\left\langle 0\left|M_{j^{\prime}}^{i^{\prime}} M_{l^{\prime}}^{k^{\prime}} \hat{\mathcal{G}}_{+} M_{j}^{\dagger i} M_{l}^{\dagger k}\right| 0\right\rangle-\frac{m_{6}^{2}}{12 f_{6}^{2}}\left\langle 0\left|M_{j^{\prime}}^{i^{\prime}} M_{l^{\prime}}^{k^{\prime}} \hat{\mathcal{G}}_{\mathcal{M}} M_{j}^{\dagger i} M_{l}^{\dagger k}\right| 0\right\rangle, \\
\mathcal{H}_{-}^{\mathrm{SU}(6)} & =\frac{u-t}{4 f_{6}^{2}}\left(\mathcal{G}_{d}-\mathcal{G}_{c}\right),
\end{aligned}
$$

where

$$
\hat{\mathcal{G}}_{+}=: \frac{1}{2} \operatorname{Tr}\left(\left[M^{\dagger}, M\right]^{2}\right):, \quad \hat{\mathcal{G}}_{\mathcal{M}}=: \operatorname{Tr}\left(\left(M+M^{\dagger}\right)^{4}\right):
$$

\footnotetext{
${ }^{4}$ Matrices, $A_{j}^{i}$, in the dimension 6 space are constructed as a direct product of flavor and spin matrices. Thus, an SU(6) index $i$, should be understood as $i \equiv(\alpha, \sigma)$, with $\alpha=1,2,3$ and $\sigma=1,2$ running over the (fundamental) flavor and spin quark degrees of freedom, respectively.

5 Our convention is such that $\left(\begin{array}{c}\bar{d} \\ \bar{u}\end{array}\right)$ is a standard basis of $\mathrm{SU}(2)$, that is, $\bar{d}=|1 / 2,1 / 2\rangle$ and $\bar{u}=|1 / 2,-1 / 2\rangle$. Thus, $\bar{u}, \bar{d}, \bar{s}$ is a standard basis of the $3^{*}$ representation of $\mathrm{SU}(3)$ with the Swart's convention [90].
} 
and

$$
\begin{aligned}
\left\langle 0\left|M_{j^{\prime}}^{i^{\prime}} M_{l^{\prime}}^{k^{\prime}} \hat{\mathcal{G}}_{+} M_{j}^{\dagger i} M_{l}^{\dagger k}\right| 0\right\rangle= & M_{j^{\prime}}^{i^{\prime}} M_{l^{\prime}}^{k^{\prime}} \operatorname{Tr}\left(\left[M^{\dagger}, M\right]\left[M^{\dagger}, M\right]\right) M_{j}^{\dagger i} M_{l}^{\dagger k} \\
& +M_{j^{\prime}}^{i^{\prime}} M_{l^{\prime}}^{k^{\prime}} \operatorname{Tr}\left(\left[M^{\dagger}, M\right]\left[M^{\dagger}, M\right]\right) M_{j}^{\dagger i} M_{l}^{\dagger k} \\
\equiv & \mathcal{G}_{d}+\mathcal{G}_{c} .
\end{aligned}
$$

In these expressions $s=\left(q_{1}+q_{2}\right)^{2}, t=\left(q_{1}-q_{3}\right)^{2}, u=\left(q_{1}-q_{4}\right)^{2},|0\rangle$ is the hadron vacuum state and : $\cdots:$ denotes the normal product.

For a fully SU(6) symmetric theory and because of Bose statistics, the interaction must be symmetric under the simultaneous exchange $(i, j) \leftrightarrow(k, l)$ and $q_{1} \leftrightarrow q_{2}$ or $\left(i^{\prime}, j^{\prime}\right) \leftrightarrow\left(k^{\prime}, l^{\prime}\right)$ and $q_{3} \leftrightarrow q_{4}$. This can be realized in two different manners: i) being both symmetric in flavor and momentum spaces, or ii) being both antisymmetric in flavor and momentum spaces. This corresponds to the decomposition $\mathcal{H}^{\mathrm{SU}(6)}=\mathcal{H}_{+}^{\mathrm{SU}(6)}+\mathcal{H}_{-}^{\mathrm{SU}(6)}$. The first of the amplitudes turns out to be purely $s$-wave, while $\mathcal{H}_{-}^{\mathrm{SU}(6)}$ describes $p$-wave scattering when mesons are degenerate in mass.

In terms of $\mathrm{SU}(6)$ projectors, the above amplitudes read (see Appendix $\mathrm{B}$ for details)

$$
\begin{aligned}
\mathcal{H}_{+}^{\mathrm{SU}(6)}= & \frac{1}{6 f^{2}}\left(3 s-\sum_{i=1}^{4} q_{i}^{2}\right)\left(-12 P_{\mathbf{1}}-6 P_{\mathbf{3 5}_{s}}-2 P_{\mathbf{1 8 9}}+2 P_{\mathbf{4 0 5}}\right) \\
& -\frac{m_{6}^{2}}{3 f^{2}}\left(23 P_{\mathbf{1}}+10 P_{\mathbf{3 5}_{s}}-2 P_{\mathbf{1 8 9}}+2 P_{\mathbf{4 0 5}}\right) \\
\mathcal{H}_{-}^{\mathrm{SU}(6)}= & 3 \frac{u-t}{f^{2}} P_{\mathbf{3 5}_{a}} .
\end{aligned}
$$

We will not discuss in this work the $p$-wave part, and we will focus here on the $s$-wave amplitude. However, $\mathcal{H}_{-}^{\mathrm{SU}(6)}$ will lead to a non-vanishing $s$-wave contribution for pseudoscalar-vector meson scattering when SU(6) symmetry breaking mass terms are considered, since in that case $(u-t)$ provides a non zero projection into $s$-wave. We return to this point below.

\section{SU(6) spin-flavor symmetry breaking effects}

The SU(6) spin-flavor symmetry is severely broken in nature. Certainly it is mandatory to take into account mass breaking effects by using different pseudoscalar and vector mesons masses. However, this cannot be done by just using these masses in the kinematics of the amplitudes derived in the previous subsection as this would lead to flagrant violations of the soft pion theorems in the $P V \rightarrow P V$ sector due to the large vector meson masses. Instead, the proper mass terms have to be added to the Lagrangian to give different mass to pseudoscalars and vectors mesons while preserving, or softly breaking, chiral symmetry. In addition, $\mathrm{SU}(2)_{\text {spin }}$ invariance must also be maintained since in the $s$-wave sector it is equivalent to angular momentum conservation.

To this end, we consider the following mass term (which replaces that in Eq. (22))

$$
\mathcal{L}_{\mathrm{SU}(6)}^{(m)}=\frac{f_{6}^{2}}{4} \operatorname{Tr}\left(\mathcal{M}\left(U_{6}+U_{6}^{\dagger}-2\right)\right)+\frac{f_{6}^{2}}{32} \operatorname{Tr}\left(\mathcal{M}^{\prime}\left(\vec{\sigma} U_{6} \vec{\sigma} U_{6}^{\dagger}+\vec{\sigma} U_{6}^{\dagger} \vec{\sigma} U_{6}-6\right)\right) .
$$

Here the matrix $\mathcal{M}$ acts only in flavor space and is to be understood as $\mathcal{M} \otimes I_{2 \times 2}$, and similarly for $\mathcal{M}^{\prime}$, so that $\mathrm{SU}(2)_{\text {spin }}$ invariance is preserved. Besides, these matrices should be diagonal in the isospin basis of Eq. (2) so that charge is conserved. Also, $\vec{\sigma}$ stands for $I_{3 \times 3} \otimes \vec{\sigma}$.

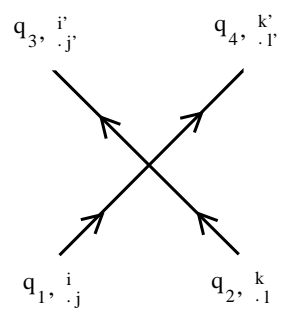

FIG. 1: Diagrammatic representation of four meson scattering in $\mathrm{SU}(6)$. 
The first term in $\mathcal{L}_{\mathrm{SU}(6)}^{(m)}$ is fairly standard. It preserves spin-flavor symmetry when $\mathcal{M}$ is proportional to the identity matrix and introduces a soft breaking of chiral symmetry when $\mathcal{M}$ is small. As it is shown below this term gives the same mass to pseudoscalar and vector mesons multiplets. Note that terms of this type are sufficient to give different mass to pseudoscalars (e.g. $\pi$ and $K$ ) when $\mathrm{SU}\left(N_{F}\right)$ is embedded into $\mathrm{SU}\left(N_{F}^{\prime}\right)$ (a larger number of flavors). They are not sufficient however to tailor different $P$ and $V$ masses when $\mathrm{SU}\left(N_{F}\right)$ is embedded into $\mathrm{SU}\left(2 N_{F}\right)$ (spin-flavor).

The second term in $\mathcal{L}_{\mathrm{SU}(6)}^{(m)}$ only gives mass to the vector mesons: indeed, if one would retain in $U_{6}$ only the pseudoscalar mesons, $U_{6}$ would cancel with $U_{6}^{\dagger}$ (since these matrices would commute with $\vec{\sigma}$ ) resulting in a cancellation of the whole term. This implies that this term does not contain contributions of the form $P P$ (pseudoscalar mass terms) nor $P P P P$ (purely pseudoscalar interaction). In addition, when $\mathcal{M}^{\prime}$ is proportional to the identity matrix (i.e., exact flavor symmetry) chiral symmetry is also exactly maintained, because the chiral rotations of $U_{6}$ commute with $\vec{\sigma}$. This guarantees that this term will produce the correct $P V \rightarrow P V$ contributions to ensure the fulfillment of soft pion WT theorem [67, 68] even when the vector mesons masses are not themselves small. ${ }^{6}$

Expanding to order $\Phi_{6}^{2}$ to isolate the genuine mass terms involved, we find

$$
\begin{aligned}
\operatorname{Tr}\left(\mathcal{M}\left(U_{6}+U_{6}^{\dagger}-2\right)\right) & =-\frac{2}{f_{6}^{2}} \operatorname{Tr}\left(\mathcal{M} \Phi_{6}^{2}\right)+\mathcal{O}\left(\Phi_{6}^{4}\right)=-\frac{2}{f_{6}^{2}} \operatorname{Tr}\left(\mathcal{M}\left(\Phi_{P}^{2}+\Phi_{V}^{2}\right)\right)+\mathcal{O}\left(\Phi_{6}^{4}\right), \\
\operatorname{Tr}\left(\mathcal{M}^{\prime}\left(\vec{\sigma} U_{6} \vec{\sigma} U_{6}^{\dagger}-3\right)\right) & =\frac{1}{f_{6}^{2}} \operatorname{Tr}\left(\mathcal{M}^{\prime}\left[\vec{\sigma}, \Phi_{6}\right]\left[\vec{\sigma}, \Phi_{6}\right]\right)+\mathcal{O}\left(\Phi_{6}^{3}\right)=-\frac{8}{f_{6}^{2}} \operatorname{Tr}\left(\mathcal{M}^{\prime} \Phi_{V}^{2}\right)+\mathcal{O}\left(\Phi_{6}^{3}\right) .
\end{aligned}
$$

Therefore

$$
\mathcal{L}_{\mathrm{SU}(6)}^{(m)}=-\frac{1}{2} \operatorname{Tr}\left(\mathcal{M} \Phi_{P}^{2}\right)-\frac{1}{2} \operatorname{Tr}\left(\left(\mathcal{M}+\mathcal{M}^{\prime}\right) \Phi_{V}^{2}\right)+\mathcal{O}\left(\Phi_{6}^{4}\right) .
$$

As advertised, $\mathcal{M}$ is the only source of mass for the pseudoscalars and so $\mathcal{M}=\operatorname{diag}\left(m_{\pi}^{2}, m_{\pi}^{2}, 2 m_{K}^{2}-m_{\pi}^{2}\right) I_{2 \times 2}$ is the usual SU(3) mass breaking matrix. On the other hand, vector mesons pick up a contribution to their mass from both $\mathcal{M}$ and $\mathcal{M}^{\prime}$.

For simplicity, in this exploratory work we will neglect the chiral breaking mass term $(\mathcal{M}=0)$ and take a common mass, $m_{V}$, for all vector mesons $\left(\mathcal{M}^{\prime}=m_{V}^{2} I_{3 \times 3} I_{2 \times 2}\right)$. We use a vector meson nonet averaged mass value $m_{V}=856$ $\mathrm{MeV}$. Let us stress that the simplifying choice $\mathcal{M}=0, \mathcal{M}^{\prime}=m_{V}^{2}$, refers only to the interaction terms derived from the Lagrangian $\mathcal{L}_{\mathrm{SU}(6)}^{(m)}$. For the evaluation of the kinematical thresholds of different channels we use physical meson masses.

With the abovementioned choice, picking up the terms of $\mathcal{O}\left(\Phi_{6}^{4}\right)$ in $\mathcal{L}_{\mathrm{SU}(6)}^{(m)}$ yields the following four-meson interaction terms

$$
\mathcal{L}_{\mathrm{SU}(6)}^{(m ; \mathrm{int})}=\frac{m_{V}^{2}}{8 f^{2}} \operatorname{Tr}\left(\Phi_{6}^{4}+\vec{\sigma} \Phi_{6}^{2} \vec{\sigma} \Phi_{6}^{2}-\frac{4}{3} \vec{\sigma} \Phi_{6} \vec{\sigma} \Phi_{6}^{3}\right) .
$$

As noted above, this Lagrangian contains only $P P V V$ and $V V V V$ interactions and no $P P P P$ ones. In addition, the $P P V V$ terms are consistent with soft pion (or soft Nambu-Goldstone boson) theorems.

Altogether, the amplitude, $\mathcal{H}$, of the process depicted in Fig. 1 after projecting into $s$-wave and for massless pseudoscalar bosons and equal mass vector mesons, takes the form

$$
\mathcal{H}=\left(\mathcal{H}_{+}^{\mathrm{SU}(6)}+\delta \mathcal{H}_{+}^{\mathrm{SU}(6)}+\mathcal{H}_{-}^{\mathrm{SU}(6)}\right)_{\mathcal{M}=0, \mathcal{M}^{\prime}=m_{V}^{2}}=\frac{1}{6 f^{2}}\left(3 s-\sum_{i=1}^{4} q_{i}^{2}\right) \mathcal{D}_{\text {kin }}+\frac{m_{V}^{2}}{8 f^{2}} \mathcal{D}_{m}+\frac{1}{2 f^{2}} \frac{m_{V}^{4}}{s} \mathcal{D}_{a} .
$$

Here $\mathcal{D}_{\text {kin }}=-12 P_{\mathbf{1}}-6 P_{\mathbf{3 5}_{s}}-2 P_{\mathbf{1 8 9}}+2 P_{\mathbf{4 0 5}}$. It accounts for the first (kinetic) term in Eq. (34), which reduces to the chirally invariant interaction proportional to $\hat{H}_{1}$ in Eq. (4). $\mathcal{D}_{m}$ is a matrix in spin-flavor space determined by the interaction $\mathcal{L}_{\mathrm{SU}(6)}^{(m \text {;int })}$ of Eq. (39). This matrix is identically zero in the $P P \rightarrow P P$ subspace and it cannot be expressed as a sum of $\mathrm{SU}(6)$ projectors, since $\mathcal{L}_{\mathrm{SU}(6)}^{(m ; \text { int })}$ breaks spin-flavor symmetry, though it is of course $Y I J$ block diagonal.

The last matrix, $\mathcal{D}_{a}$, is just $6 P_{\mathbf{3 5}_{a}}$ in the $P V \rightarrow P V$ sector and zero otherwise. $\mathcal{H}_{-}^{\mathrm{SU}(6)}$ of Eq. (35) has now a nonvanishing contribution and once again, neglecting these terms would introduce a large violation of chiral symmetry,

\footnotetext{
${ }^{6}$ Under a chiral transformation, the vector meson mass term $\left.\left(m_{V}^{2} \operatorname{Tr}\left(\Phi_{V}^{2}\right)\right)\right)$ will give rise to a $P V V$ term which can only get canceled by the corresponding variation of the $P P V V$ contact term (recall $\delta P \sim \mathcal{O}(1)$ and $\delta V \sim \mathcal{O}(P V, V V)$ ) to ensure that the whole Lagrangian is invariant. Thus the contact $P P V V$ piece is fixed by chiral symmetry.
} 
proportional to the vector meson mass. This comes about because $(u-t)$ gives rise to non-zero $s-$ wave contributions for $P V \rightarrow P V$ scattering, once pseudoscalar-vector mass breaking is taken into account. Indeed, in the limit of massless Nambu-Goldstone bosons and mass degenerated vector mesons, one finds for the $P V$ sector (assuming that the legs 1 and 3 in Fig. 1 are of type $P$ and 2 and 4 of type $V$ )

$$
\langle u-t\rangle_{l=0}=\frac{m_{V}^{4}}{s} \quad(P V \rightarrow P V) .
$$

The same average vanishes for $P P$ or $V V$ sectors, since there Bose symmetry still applies.

Regarding the fulfillment of the relations Eqs. (15,19) in subsection IIB, we can see that $\mathcal{H}$ of Eq. (40) reduces to

$$
\mathcal{H}_{P P \rightarrow P P}=\frac{s}{2 f^{2}} \mathcal{D}_{\text {kin }}
$$

in the $P P$ sector. On the other hand, in the $P V$ sector, the relation

$$
\frac{1}{3} \mathcal{D}_{\text {kin }}+\frac{1}{4} \mathcal{D}_{m}+\mathcal{D}_{a}=0 \quad(P V \rightarrow P V \text { sector })
$$

guarantees that $\mathcal{H}(s)$ vanishes at threshold in this sector, and moreover

$$
\mathcal{H}_{P V \rightarrow P V}=\frac{\left(s-m_{V}^{2}\right)}{2 f^{2}}\left(\mathcal{D}_{\text {kin }}-\frac{m_{V}^{2}}{s} \mathcal{D}_{a}\right) .
$$

These expressions fulfill the relations Eqs. (15]19]) by taking $F_{\mathcal{R}}(s)=s-\frac{1}{2} \sum_{i=1}^{4} q_{i}^{2}$ for the symmetric representations and $F_{\mathcal{R}}(s)=\left(s-\frac{1}{2} \sum_{i=1}^{4} q_{i}^{2}\right) m_{V}^{2} / s$ for the antisymmetric ones.

We have also considered spin-flavor symmetry breaking effects due to the difference between pseudoscalar and vector meson decay constants. The pseudoscalar meson decay constants, $f_{P}$, are defined by

$$
\left\langle 0\left|\bar{q}_{1} \gamma^{\mu} \gamma_{5} q_{2}(0)\right| P(p)\right\rangle=-\mathrm{i} \sqrt{2} f_{P} p^{\mu}
$$

and vector meson decay constants, $f_{V}$, by

$$
\left\langle 0\left|\bar{q}_{1} \gamma^{\mu} q_{2}(0)\right| V(p, \epsilon)\right\rangle=\sqrt{2} m_{V} f_{V} \epsilon^{\mu},
$$

where $\bar{q}_{1}, q_{2}$ are the quark fields, $\epsilon_{\mu}$ is the polarization vector of the meson, and $m_{V}$ its mass. With the above definitions, in the limit where either the quark or the antiquark that forms the meson becomes infinitely heavy and thus spin symmetry turns out to be exact, QCD predicts $f_{P}=f_{V}$ [99]. This guarantees that the normalizations of the coupling constants in Eqs. (45) and (46) are consistent. For light mesons there exist sizable corrections to the heavy quark symmetry-type relation $f_{P}=f_{V}$. For instance, the ratio $f_{\rho} / f_{\pi}$ is of the order of 1.7 . To take this into account, in Eq. (40) we apply the prescription

$$
\frac{1}{f^{2}} \rightarrow \frac{1}{\left(f_{1} f_{2} f_{3} f_{4}\right)^{1 / 2}}
$$

where the labels $1,2,3,4$ refer the four interacting mesons.

The meson decay constants (taken from Ref. [65]) and masses used throughout this work to compute the kinematical thresholds and loop functions are compiled in Table II while the coupled-channel matrices $\mathcal{D}_{\text {kin }}, \mathcal{D}_{m}$ and $\mathcal{D}_{a}$ can be found in Tables XXI LXI of Appendix A. We assume an ideal mixing in the vector meson sector, namely, $\omega=\sqrt{\frac{2}{3}} \omega_{1}+\frac{1}{\sqrt{3}} \omega_{8}$ and $\phi=\sqrt{\frac{2}{3}} \omega_{8}-\frac{1}{\sqrt{3}} \omega_{1}$. The conventions of [90] are used throughout. Note that for the $Y=0$ channels, $G$-parity is conserved, ${ }^{7}$ and that all $Y=0$ states have well-defined $G$-parity except the $\bar{K}^{*} K$ and $K^{*} \bar{K}$ states, but the combinations $\left(\bar{K} K^{*} \pm K \bar{K}^{*}\right) / \sqrt{2}$ are actually $G$-parity eigenstates with eigenvalues \pm 1 . These states will be denoted $\left(\bar{K} K^{*}\right)_{S}$ and $\left(\bar{K} K^{*}\right)_{A}$, respectively.

A final remark is in order here. The new model introduced in this work is given by $\mathcal{L}_{\mathrm{SU}(6)}^{\mathrm{kin}}+\mathcal{L}_{\mathrm{SU}(6)}^{(m)}($ namely, the first term in Eq. (22) and that in Eq. (36)). It implements the approximate spin-flavor chiral symmetry (as opposed

\footnotetext{
${ }^{7}$ Recall that the $G$-parity operation can be defined through its action on an $Y I I_{3}$ eigenstate as $G\left|Y I I_{3}\right\rangle=\chi(-1)^{Y / 2+I}\left|-Y I I_{3}\right\rangle$, with $\chi$ the charge conjugation of a neutral non-strange member of the $\mathrm{SU}(3)$ family.
} 
to the standard flavor chiral symmetry) advocated by Caldi and Pagels [79, 80]. In their approach vector mesons are identified as dormant Nambu-Goldstone bosons. $\mathcal{L}_{\mathrm{SU}(6)}^{\text {kin }}$ displays such extended chiral symmetry, while $\mathcal{L}_{\mathrm{SU}(6)}^{(m)}$ models the pattern of symmetry breaking. Regarding this latter term, it should be noted that there is a large ambiguity in choosing it. Being a contact term, it cannot contain PPPP contributions, due to chiral symmetry, and for the same reason the terms $P P V V$ are also fixed, as already noted. However, $V V V V$ terms are not so constrained. One can easily propose alternative forms for $\mathcal{L}_{\mathrm{SU}(6)}^{(m)}$ which would still be acceptable from general requirements but would yield different $V V V V$ interactions. For instance, any term of the form $\operatorname{Tr}\left(\mathcal{M} \vec{\sigma} U_{6} \vec{\sigma} U_{6}^{\dagger} \vec{\sigma} U_{6} \vec{\sigma} U_{6}^{\dagger} \ldots\right)$, with the indices of the $\vec{\sigma}$ matrices contracted in any order, could be present in $\mathcal{L}_{\mathrm{SU}(6)}^{(m)}$. Our choice in Eq. (36) is just the simplest or minimal one. ${ }^{8}$ Of course, such minimal choices are also present in any other model, often tied to some expansion parameter. We have not yet identified a hierarchy to choose among the various available operators. Ultimately, the ambiguity should be fixed by requiring consistency with the asymptotic behavior of QCD [95]. In what follows we will present results obtained with the interaction $\mathcal{H}$ given in Eq. (40).

\section{BS MESON-MESON SCATTERING AMPLITUDE}

To describe the dynamics of resonances one needs to have exact elastic unitarity in coupled-channel. For that purpose, we solve the coupled-channel BS equation and use the $\mathrm{SU}(6)$ broken potential defined above to construct its interaction kernel. In this way in any $Y I J$ sector, the solution for the coupled-channel $s$-wave scattering amplitude, $T^{Y I J}$, satisfies exact unitarity in coupled-channel. In the so called on-shell scheme [23, 24, 28, 36], $T^{Y I J}$ is given by

$$
T^{Y I J}(s)=\frac{1}{1-V^{Y I J}(s) G^{Y I J}(s)} V^{Y I J}(s) .
$$

$V^{Y I J}(s)$ (a matrix in coupled-channel space) stands for the projection of the scattering amplitude, $\mathcal{H}$, in the $Y I J$ sector. $G^{Y I J}(s)$ is the loop function and is diagonal in the coupled-channel space. Suppressing the indices, it is written for each channel as

$$
G(s)=i \int \frac{d^{4} q}{(2 \pi)^{4}} \frac{1}{q^{2}-m_{1}^{2}} \frac{1}{(P-q)^{2}-m_{2}^{2}}
$$

where $m_{1}$ and $m_{2}$ are the masses of the mesons corresponding to the channel, for which we take physical values, and $P^{\mu}$ is the total four momentum $\left(P^{2}=s\right)$. The loop function involves a logarithmic ultraviolet divergence which needs to be dealt with. Extracting a suitable infinite constant, one can write

$$
G(s)=\bar{G}(s)+G\left(\left(m_{1}+m_{2}\right)^{2}\right) .
$$

The finite function $\bar{G}(s)$ can be found in Eq. (A9) of Ref. [32], and it displays the unitarity right-hand cut of the amplitude. On the other hand, the constant $G\left(\left(m_{1}+m_{2}\right)^{2}\right)$ contains the logarithmic divergence. After renormalizing using the dimensional regularization scheme, one finds

$$
G\left(s=\left(m_{1}+m_{2}\right)^{2}\right)=\frac{1}{16 \pi^{2}}\left(a(\mu)+\frac{1}{m_{1}+m_{2}}\left\{m_{1} \ln \frac{m_{1}^{2}}{\mu^{2}}+m_{2} \ln \frac{m_{2}^{2}}{\mu^{2}}\right\}\right)
$$

where $\mu$ is the scale of the dimensional regularization. Changes in the scale are reabsorbed in the subtraction constant $a(\mu)$, so that the results remain scale independent.

We fix the Renormalization Scheme (RS) used in this work as follows. We adopt a reasonable scale $\mu=1 \mathrm{GeV}$ and we allow $a(\mu)$ to vary around the value -2 to best describe the known phenomenology in each $Y I J$ sector. ${ }^{9}$ Results,

\footnotetext{
8 As it turns out, the same term has been proposed by Caldi 98 as a Lorentz symmetry restoration correction.

9 One can instead use an ultraviolet hard cutoff $\Lambda$ to renormalize the loop function. The relation between the subtraction constant $a(\mu)$, at the scale $\mu$, and $\Lambda$ is

$$
a(\mu)=-\frac{2}{m_{1}+m_{2}}\left\{m_{1} \ln \left[\frac{\Lambda+\sqrt{\Lambda^{2}+m_{1}^{2}}}{\mu}\right]+m_{2} \ln \left[\frac{\Lambda+\sqrt{\Lambda^{2}+m_{2}^{2}}}{\mu}\right]\right\}
$$

For $\mu=0.7-1 \mathrm{GeV}$, and assuming a cutoff of the same order of magnitude, -2 turns out to be a natural choice for the subtraction
} constant $a(\mu)$. 
TABLE II: Values for the meson masses and decay constants used in the numerical calculations. All units are in MeV. Besides, we use $m_{V}=856 \mathrm{MeV}$ as parameter of the Lagrangian.

\begin{tabular}{cc|cc}
\hline \hline$m_{\pi}$ & 138.0 & $f_{\pi}$ & 92.4 \\
$m_{K}$ & 495.7 & $f_{K}$ & 113.0 \\
$m_{\eta}$ & 547.5 & $f_{\eta}$ & $1.2 \times f_{\pi}$ \\
$m_{\rho}$ & 775.5 & $f_{\rho}$ & 153 \\
$m_{K^{*}}$ & 893.8 & $f_{K^{*}}$ & 153 \\
$m_{\omega}$ & 782.7 & $f_{\phi}$ & 163 \\
$m_{\phi}$ & 1019.5 & $f_{\omega}$ & $f_{\rho}$ \\
\hline \hline
\end{tabular}

of course, have some dependence on the adopted RS, as they also depend on the assumed SU(6) breaking pattern of the couplings $\left(1 / f^{2} \rightarrow 1 /\left(f_{1} f_{2} f_{3} f_{4}\right)^{1 / 2}\right)$. Indeed, both choices are not independent from each other. That is the reason why we do not mind to scale, for instance, the $\pi \rho \rightarrow \pi \rho$ channel by $1 /\left(f_{\pi} f_{\rho}\right)$ instead of by $1 / f_{\pi}^{2}$, as one will naturally expect from chiral symmetry [97], since a change in the renormalization scale or in the subtraction constant for this channel would easily cover the differences among these two choices for the couplings.

Since $f_{V}$ is significantly higher than $f_{P}$, the adopted breaking pattern for the couplings guarantees that low-lying $J^{P}=0^{+}$resonances, such as the $f_{0}(980)$ or the $f_{0}(600)$, described previously by unitarizing pseudoscalar-pseudoscalar meson amplitudes [18, 19, 22, 30] are not much affected by the inclusion of vector-vector meson channels. It will be shown below that the adopted RS successfully describes the main features of these positive parity scalar resonances.

Other on-shell renormalization schemes can be also adopted. For instance, one can take a certain scale, $\mu$, such that $G\left(\mu^{2}\right)=0$ and the $T^{Y I J}$-amplitude reduces to the two-particle irreducible amplitude $V^{Y I J}$, i.e., $T^{Y I J}\left(\mu^{2}\right)=$ $V^{Y I J}\left(\mu^{2}\right)$. This fixes the value of the subtraction constant $G\left(\left(m_{1}+m_{2}\right)^{2}\right)$. This approach has been adopted in [36, 41, 44, 46, 65] for meson-baryon $s$-wave scattering. The use of one RS or another is part of the uncertainties of the present approach, though, they are smaller than those associated to our incomplete knowledge of the two-particle irreducible amplitude $V^{Y I J}$. We do not expect large differences in the gross features of the picture that emerges, though the exact position of the poles can of course be affected by modifying the RS. In the present work, the use of the RS based on dimensional regularization, as outlined above, is preferable, because the same RS has been adopted in previous studies of vector meson-vector meson $(V V)$ and pseudoscalar meson-vector meson $(P V)$ scattering within the hidden gauge unitary approach [43, 69, 70]. This makes it easier to compare our results with those obtained in these references.

\section{RESULTS AND DISCUSSION}

In this section, we show the results obtained using the approach described above and compare them with those obtained earlier within different schemes, and to data when possible.

The mass and widths of the dynamically generated resonances in each $Y I J$ sector are determined from the positions of the poles, $s_{R}$, in the Second Riemann Sheet (SRS) of the corresponding scattering amplitudes, namely $s_{R}=$ $M_{R}^{2}-\mathrm{i} M_{R} \Gamma_{R}$. For narrow resonances $\left(\Gamma_{R} \ll M_{R}\right), \sqrt{s_{R}} \sim M_{R}-\mathrm{i} \Gamma_{R} / 2$ constitutes a good approximation. In some cases, we also find real poles in the First Riemann Sheet (FRS) of the amplitudes which correspond to bound states.

The coupling constants of each resonance to the various meson-meson states are obtained from the residues at the pole, by matching the amplitudes to the expression

$$
T_{i j}^{Y I J}(s)=\frac{g_{i} g_{j}}{\left(s-s_{R}\right)},
$$

for energy values $s$ close to the pole. The couplings, $g_{i}$, are complex in general.

Since our starting point is the chiral dynamics governing the interaction among Nambu-Goldstone bosons, low energy results should be similar to those previously obtained by unitarizing one loop ChPT amplitudes [18, 19, 30]. Because of the inclusion of vector meson degrees of freedom the scalar sector has an enlarged coupled-channel space in our case. However, we expect small effects from these new degrees of freedom on the low-lying scalar resonances, since 
vector meson-vector meson thresholds are relatively far away from the low-energy region, where the pseudoscalarpseudoscalar interaction dominates.

To facilitate the discussion of our results, let us point out the main differences between the approach advocated in the present work and the approaches followed in Ref. [43] for the pseudoscalar-vector sector, and in Refs. 69, 70] for the vector-vector one. These latter works are based ${ }^{10}$ on the formalism of the hidden gauge interaction for vector mesons [74, 75]. The main differences are:

1. Previous works [43, 69, 70] treat separately pseudoscalar-pseudoscalar, pseudoscalar-vector and vector-vector meson sectors. However, for instance, vector-vector channels could modify the properties of some vector-axial resonances, generated in Ref. [43], where only pseudoscalar-vector meson interactions are considered. Within the formalism of the hidden gauge interaction for vector mesons there exist no $s$-wave $P V \rightarrow V V$ transition potentials at tree level, and thus it is difficult to overcome this limitation in that scheme.

2. Pseudoscalar-vector channels [43]: Though, in a first view, the two-particle irreducible amplitude $\left(V^{Y I J}\right)$ employed here and that used in [43] might look quite different, this is not really the case and they just differ at order $\mathcal{O}\left(m^{2}, \vec{k}^{2}\right)$ (with $m$ and $k^{\mu}$, the mass and the momentum of the Nambu-Goldstone boson) in the chiral expansion, which is not fixed by the LO WT theorem [67, 68] that both approaches satisfy. Thus, in both schemes, the potentials $V^{Y I J}$ totally agree at LO $\mathcal{O}\left(k^{\mu}\right)$ and take the common value

$$
V^{Y I J}=C^{Y I J} m_{V} k^{0} / f^{2}
$$

where the $C^{Y I J}$ coupled-channel matrices are given in [43]. The $P V \rightarrow P V$ amplitudes vanish in the soft Nambu-Goldstone boson limit $k^{0} \rightarrow 0$, as required by the LO WT theorem (see discussion in [85] for some more details).

As a consequence, and apart from the influence of the vector meson-vector meson channels (see point below), of the use here of massless Nambu-Goldstone bosons and physical decay constants in the computation of $V^{Y I J}$, we expect a rather good agreement with the results of Ref. [43] for the lowest lying axial resonances, which will not be much affected by higher orders of the chiral expansion.

3. Vector-vector channels [69, 70]: In Refs. [69, 70], contact, box and $t$ - and $u$-exchange contributions were considered, within a scheme based on the hidden gauge interaction for vector mesons; the exchange and contact terms being the dominant mechanisms. The exchange mechanism is closely related to the kinetic interaction derived within our $\mathrm{SU}(6)$ symmetric scheme $\left(\mathcal{D}_{\text {kin }}\right)$. Indeed, one finds that by symmetrizing the interaction in the $\rho \rho$ channel in Table I of Ref. 69] and adding a factor 4/3, our SU(6) symmetric $\rho \rho$ interaction is reproduced $\left(\mathcal{D}_{\text {kin }}\right.$ can be looked up in Appendix A). Note that in the $\rho \rho$ channel SU(6) symmetry implies having symmetric interactions under the exchange $I \leftrightarrow J$.

As we commented above, the kinetic interaction of our model is of the form $\left[(\mathbf{3 5} \otimes \mathbf{3 5})_{\mathbf{3 5}} \otimes(\mathbf{3 5} \otimes \mathbf{3 5})_{\mathbf{3 5}}\right]_{\mathbf{1}}$ in the $t$-channel. This can be regarded as the zero-range $t$-channel exchange of a full $\mathbf{3 5}$ irreducible representation, carried by an octet of spin 0 and a nonet of spin 1 mesons of even parity. In Refs. 69, 70] these kinetic terms are originated by the $t$-exchange of the time component of vector mesons, which has certain resemblance with our zero-range exchange of $0^{+}$mesons. Parity and angular momentum conservation also allow the exchange of $1^{+}$and $2^{+}$mesons. The latter exchange is missing in both approaches, and the former one is included within our scheme, as required by $\mathrm{SU}(6)$ symmetry, while it is not present in the hidden gauge formalism adopted in Refs. [69, 70]. We do not see a priori any compelling reason to favor any of the two approaches.

The contact terms in both approaches seem to be totally unrelated. We remind here the ambiguities mentioned above associated to this term and that presumably its actual nature can only be fixed by the asymptotic behavior of QCD.

4. We use $f_{V} \neq f_{P}$ for those channels which involve vector mesons, while a universal $1 / f^{2}$ coupling is assumed for all channels in the previous works. As commented above, this is somehow related with the RS.

In what follows, we show results for the different $Y I J$ sectors, considering only nonnegative hypercharge values.

10 Strictly speaking, the study of axial-vector resonances carried out in Ref. [43] does not use the hidden gauge formalism. There, a contact WT type Lagrangian is employed. However, the tree level amplitudes so obtained coincide with those deduced within the hidden gauge formalism, neglecting $q^{2} / m_{V}^{2}$ in the $t$-exchange contributions [101] and considering only the propagation of the time component of the virtual vector mesons. 
TABLE III: Pole positions and modulus of the couplings $|g|(\mathrm{MeV}$ units $)$ in the $(Y, I, J)=(0,0,0)$ sector. $I^{G}\left(J^{P C}\right)=0^{+}\left(0^{++}\right)$. The subtraction constant has been set to its default value, $a=-2$. Possible PDG counterparts: $f_{0}(600), f_{0}(980), f_{0}(1370)$, and $f_{0}(1710)$.

\begin{tabular}{c|cccccccc}
\hline \hline$\sqrt{s_{R}}$ & $\pi \pi$ & $\bar{K} K$ & $\eta \eta$ & $\rho \rho$ & $\omega \omega$ & $\omega \phi$ & $\bar{K}^{*} K^{*}$ & $\phi \phi$ \\
\hline$(635,-202)$ & 3516 & 432 & 333 & 7592 & 7909 & 117 & 7306 & 1850 \\
$(969,0)$ & 28 & 2983 & 2477 & 3393 & 2401 & 1627 & 4305 & 3831 \\
$(1350,-62)$ & 553 & 3257 & 840 & 1336 & 2841 & 7074 & 10697 & 10647 \\
$(1723,-52)$ & 43 & 853 & 3154 & 318 & 408 & 3400 & 2470 & 13698 \\
\hline \hline
\end{tabular}

TABLE IV: Pole positions and the modulus of the couplings (MeV units) in the $(Y, I, J)=(0,0,0)$ sector when only block diagonal pseudoscalar-pseudoscalar and vector-vector meson interactions are used. $I^{G}\left(J^{P C}\right)=0^{+}\left(0^{++}\right)$. Possible PDG counterparts: $f_{0}(600), f_{0}(980), f_{0}(1370)$ and $f_{0}(1710)$.

\begin{tabular}{c|cccccccc}
\hline \hline$\sqrt{s_{R}}$ & $\pi \pi$ & $\bar{K} K$ & $\eta \eta$ & $\rho \rho$ & $\omega \omega$ & $\omega \phi$ & $\bar{K}^{*} K^{*}$ & $\phi \phi$ \\
\hline$(485,-156)$ & 2807 & 600 & 86 & & & & & \\
$(990,-6)$ & 862 & 2746 & 2146 & & & & & \\
$(1217,0)$ & & & & 3637 & 3111 & 1378 & 12465 & 13130 \\
$(1981,-110)$ & & & & 848 & 1896 & 4984 & 3708 & 10747 \\
\hline \hline
\end{tabular}

\section{A. Hypercharge 0, isospin 0 and spin 0}

There are eight coupled channels, i.e., $\pi \pi, \bar{K} K, \eta \eta, \rho \rho, \omega \omega, \omega \phi, \bar{K}^{*} K^{*}$ and $\phi \phi$. In all cases the $G-$ parity is positive. Four poles are found on the complex plane of the SRS. These are compiled in Table III, where the modulus of the couplings to the different channels (see Eq. (53) ) are also given. The lowest two poles can be easily identified with the $f_{0}(600)$ and $f_{0}(980)$ resonances. There are some differences with other works [22, 30] mainly because we have neglected the pseudoscalar meson mass terms and have incorporated vector meson-vector meson channels. On the other hand, the identification of the other two poles is not so direct, though it is tempting to associate them to the $f_{0}(1370)$, and $f_{0}(1710)$ resonances. Thus, in our model the $f_{0}(1370)$ resonance has a sizeable coupling to the $\rho \rho$ channel which would lead to a four pion decay mode. For the decay of the resonance, the $\rho \rho$ channel is more relevant than the other ones (for instance the $\omega \omega$ or $\bar{K}^{*} K^{*}$ ), thanks to the large width of the $\rho$-meson, which enhances the decay of the resonance to the decay products of the $\rho \rho$ pair. Indeed, the width of these $f_{0}$ resonances will be enhanced when new mechanisms constructed out of VPP $p$-wave couplings are considered (see for example Fig. 21) 70]. For instance, since the pole that we have associated to the $f_{0}(1370)$ is placed below the two $\rho$ meson threshold, it can decay neither to this channel nor to those which are even heavier. Thus, the width of around $124 \mathrm{MeV}$ that can be read off from Table III accounts only for the decay of the resonance into the open channels $(\pi \pi, \bar{K} K, \eta \eta)$. However, the resonance can decay into two virtual $\rho$ mesons, and each of them subsequently will decay into two pions, giving rise to four and two pion decay modes through processes like those sketched in Fig. 2. These decays will increase the width of the resonance 70]. Obvious modifications to these mechanisms should be considered, taking into account the specific details of the dominant decays of the corresponding vector mesons, for other channels. For instance, since the $\omega$ meson decays predominantly into three pions, the coupling of a resonance to two $\omega$ mesons will produce six or four pion decays.

Following the findings of Ref. [70], a substantial increase of both the $f_{0}(1370)$ and $f_{0}(1710)$ widths with respect to those deduced from the pole position is to be expected. On the other hand, the above mechanisms could explain a large $K \bar{K}$ decay mode of the $f_{0}(1710)$ resonance that in our model couples strongly to the $K^{*} \bar{K}^{*}$ and the $\phi \phi$ channels. This also supports the picture of Ref. [102], where it is guessed that the $f_{0}(1710)$ is dominantly $s \bar{s}$. Besides, we predict a sizeable decay of this resonance into $\eta \eta$. 
The experimental $f_{0}(1500)$, on the other hand, has a mass of $1505 \pm 6 \mathrm{MeV}$ and it is relatively narrow $(\Gamma=$ $109 \pm 7 \mathrm{MeV}$ ) with dominant decays into two and four pion channels. Owing to the above discussion, it would be difficult to assign it to our lowest pole, and thus it is a clear candidate to have a dominant glueball structure [103, 104]. This is also in agreement with the recent claims of Albaladejo and Oller [105], though it looks more difficult to reconcile the picture that emerges from our analysis with this latter work in the case of the $f_{0}(1710)$ resonance. This is because in Ref. [105], the $f_{0}(1710)$ resonance is identified as an unmixed glueball with a large $\eta^{\prime} \eta^{\prime}$ coupling, and this latter channel is not included in our scheme. In Ref. [70], only the $f_{0}(1370)$ and $f_{0}(1710)$ resonances are found as well, and in agreement with our findings, there the $f_{0}(1500)$ is not dynamically generated either. However, there appear some differences with our results, since in this latter reference the $f_{0}(1370)$ is mainly $\rho \rho$, and the $f_{0}(1710)$ is mostly $K^{*} \bar{K}^{*}$. Such a distinction is not so clear in our scheme, where $\omega \omega, \omega \phi$ and $\phi \phi$ channels play a more significant role than in the hidden gauge unitarity approach advocated in [70].
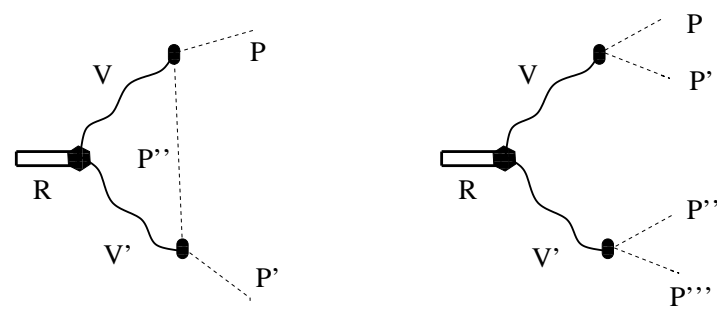

FIG. 2: Resonance $(R)$ decay to two (left) or four (right) pseudoscalar mesons $\left(P, P^{\prime}, P^{\prime \prime}, P^{\prime \prime \prime}\right)$ through its $s-$ wave (hexagon) coupling to two vector mesons $\left(V, V^{\prime}\right)$ and the $p$-wave coupling (ovals) of these latter mesons to two pseudoscalar mesons.

In previous studies of pseudoscalar-pseudoscalar and vector-vector interactions, the first three channels and the last five channels in Table [II were considered separately (see for instance Refs. [18, 19] and [70], respectively). It is interesting to check how the results change if they are also considered separately. That is, if all couplings that connect the pseudoscalar-pseudoscalar and vector-vector meson sectors are set to zero. Comparing the results of Tables [III] and IV] we observe that, though the number of resonances is the same in both cases (four) their positions and the relative strengths of couplings to different channels have changed. The inclusion of the three pseudoscalar-pseudoscalar channels has a large impact on the two poles of higher energy.

\section{B. Hypercharge 0 , isospin 0 and spin 1}

In this sector (see Table $\mathrm{V}$ ), there are two sets of quantum numbers: $I^{G}\left(J^{P C}\right)$ equal $0^{-}\left(1^{+-}\right)$and $0^{+}\left(1^{++}\right)$, corresponding to those of the $h_{1}$ and $f_{1}$ resonances, respectively. Interactions turn out to be block diagonal since strong interactions conserve $G$-parity and charge conjugation.

For $0^{-}\left(1^{+-}\right)$there are five coupled channels, namely, $\eta \phi, \eta \omega, \pi \rho,\left(\bar{K} K^{*}\right)_{A}=\frac{1}{\sqrt{2}}\left(\bar{K} K^{*}-K \bar{K}^{*}\right), K^{*} \bar{K}^{*}$. Three poles are found in the complex plane, which can be tentatively associated to the $h_{1}(1170), h_{1}(1380)$, and $h_{1}(1595)$. (These are the only three $0^{-}\left(1^{+-}\right)$resonances below $2 \mathrm{GeV}$ quoted in the PDG [84].) Naturally, huge couplings are found for the $h_{1}(1170), h_{1}(1380)$ and $h_{1}(1595)$ resonances to the $\pi \rho,\left(\bar{K} K^{*}\right)_{A}$ and $\eta \omega$ and $\bar{K}^{*} K^{*}$ channels, respectively.

TABLE V: Pole positions and modulus of the couplings $\left(\mathrm{MeV}\right.$ units) in the $(Y, I, J)=(0,0,1)$ sector. $I^{G}\left(J^{P C}\right)=0^{-}\left(1^{+-}\right)$ $\left[h^{\prime} s\right]$ and $0^{+}\left(1^{++}\right)\left[f^{\prime} s\right]$. We have slightly moved away from the choice $a=-2$ for the subtraction constants (see Eq. (51)) and have used $a=-1.3$ and $a=-2.9$ for the $0^{-}\left(1^{+-}\right)$and $0^{+}\left(1^{++}\right)$sub-sectors, respectively. Possible PDG counterparts: $h_{1}(1170), h_{1}(1380), h_{1}(1595)$ and $f_{1}(1285)$.

\begin{tabular}{c|ccccccc|c}
\hline \hline$\sqrt{s_{R}}$ & $\eta \phi$ & $\eta \omega$ & $\pi \rho$ & $\left(\bar{K} K^{*}\right)_{A}$ & $\bar{K}^{*} K^{*}$ & $\omega \phi$ & $\left(\bar{K} K^{*}\right)_{S}$ & $G$ \\
\hline$(1006,-85)$ & 52 & 26 & 4362 & 1192 & 1029 & & & - \\
$(1373,-17)$ & 2957 & 2484 & 1273 & 5322 & 1779 & & & - \\
$(1600,-67)$ & 2414 & 3655 & 1006 & 1033 & 12869 & & & - \\
$(1286,0)$ & & & & & & 4089 & 6790 & + \\
\hline \hline
\end{tabular}


While the two latter resonances are omitted from the summary PDG table, and even the isospin and $G$-parity of the $h_{1}(1380)$ is not quoted, the $h_{1}(1170)$ is firmly established experimentally. We predict for it a width smaller than that quoted in the PDG, because within our model it comes out lighter than the experimental one and thus the available phase space for $\pi \rho$ decay is much smaller.

The $h_{1}(1170)$ and $h_{1}(1380)$ obtained here are placed at similar positions and have similar couplings and widths as those obtained in Ref. [43]. This is not surprising since, as already noted, at LO in the chiral expansion our coupled-channel interaction for $P V \rightarrow P V$ scattering coincides with that used in Ref. [43].

Within our scheme, the vector-vector channel $K^{*} \bar{K}^{*}$ plays an important role in the dynamics of the pole placed at $(1600,-67) \mathrm{MeV}$. Presumably, this is the reason why a third $h_{1}$ pole was not found in Ref. [43], which misses the $K^{*} \bar{K}^{*}$ channel. On the other hand, a $K^{*} \bar{K}^{*}$ resonance is found in Ref. [70] located almost at threshold $\left[\sqrt{s_{R}}=(1802,-39)\right]$. This pole was not identified in Ref. [70] with the $h_{1}(1595)$, because of the very different mass. It can be conjectured that this pole corresponds to the one found in our approach at $(1600,-67)$. The latter is strongly modified by the inclusion of the $\omega \eta$ channel in the dynamics. If the picture presented by our model is correct, with the pole at $(1600,-67)$ assigned to $h_{1}(1595)$, this resonance cannot be generated just by $\omega \eta$, as attempted in Ref. [43]. Indeed, the diagonal $\omega \eta$ potential is zero in this sector. And also it cannot be described using only $K^{*} \bar{K}^{*}$, as in 70$]$, since the mass turns out to be too high and furthermore its dominant decay mode, $\omega \eta$, is ignored.

In the $0^{+}\left(1^{++}\right)$subsector only one pole is found, at $(1286,0)$, quite similar to that reported in Ref. [43]. The PDG quotes three $f_{1}$ resonances below $2 \mathrm{GeV}$ : $f_{1}(1285), f_{1}(1420)$, and $f_{1}(1510)$. The $f_{1}(1285)$ has a mass of $1281.8 \pm 0.6$ $\mathrm{MeV}$ and a width of $24.3 \pm 1.1 \mathrm{MeV}$; the $f_{1}(1420)$ has a mass of $1426.4 \pm 0.9 \mathrm{MeV}$ and a width of $54.9 \pm 2.6 \mathrm{MeV}$; the $f_{1}(1510)$ has a mass of $1518 \pm 5 \mathrm{MeV}$ and a width of $73 \pm 25 \mathrm{MeV}$. The decay modes of $f_{1}(1420)$ and $f_{1}(1510)$ are dominated by the $\left(K \bar{K}^{*}\right)_{S}$ mode. On the other hand, because the $f_{1}(1285)$ is below the $\bar{K} K^{*}$ threshold, it cannot decay through this channel, though the branching fraction into $\bar{K} K \pi$ is about $10 \%$ and it might hint at a non-negligible $\bar{K} K^{*}$ component in its wave function.

Because of the position of the pole at $(1286,0)$, it makes sense to assign this pole to the $f_{1}(1285)$ resonance, as it was done in Ref. [43]. The reason why no width is found for this resonance, while the PDG quotes $24 \mathrm{MeV}$ for it, is that there are other decay channels different to $V P$ that are obviously not considered in our scheme [like $4 \pi(33 \%)$, $\eta \pi \pi(52 \%)$, or $\left.K \bar{K} \pi(10 \%)^{11}\right]$. Nevertheless, the assignment of the pole to the $f_{1}(1420)$ resonance, whose dominant decay is $\left(K \bar{K}^{*}\right)_{S}$ and it is also placed close to threshold, cannot be completely discarded either.

TABLE VI: Pole positions and modulus of the couplings $(\mathrm{MeV}$ units $)$ in the $(Y, I, J)=(0,0,2)$ sector. $I^{G}\left(J^{P C}\right)=0^{+}\left(2^{++}\right)$. In this subsector the value of -2.77 for the subtraction constant in the $\rho \rho$ channel and -2.5 for the other ones have been used. Possible PDG counterparts: $f_{2}(1270)$ and $f_{2}(1640)$.

\begin{tabular}{c|ccccc}
\hline \hline$\sqrt{s_{R}}$ & $\rho \rho$ & $\omega \omega$ & $\omega \phi$ & $\bar{K}^{*} K^{*}$ & $\phi \phi$ \\
\hline$(1289,0)$ & 22138 & 17793 & 3642 & 18385 & 5865 \\
$(1783,-19)$ & 2235 & 1541 & 2846 & 5201 & 2427 \\
\hline \hline
\end{tabular}

\section{Hypercharge 0 , isospin 0 and spin 2}

In this sector, there are five coupled channels: $\rho \rho, \omega \omega, \omega \phi, K^{*} \bar{K}^{*}$, and $\phi \phi$, and we find two poles (see Table VI) in the SRS/FRS of our amplitudes. Experimentally, many $f_{2}$ resonances below $2 \mathrm{GeV}$ have been reported, including $f_{2}(1270), f_{2}(1430), f_{2}^{\prime}(1525), f_{2}(1565), f_{2}(1640), f_{2}(1810), f_{2}(1910)$, and $f_{2}(1950)$. Most of them $\left[f_{2}(1430), f_{2}(1565)\right.$, $f_{2}(1640), f_{2}(1810)$, and $f_{2}(1910)$ ] have not been confirmed yet. It is tempting to associate the two $f_{2}$ poles found within our approach to the two lowest lying confirmed resonances $f_{2}(1270)$ and the $f_{2}^{\prime}(1525)$. In the first case, the mass agrees well with that quoted in the PDG, however experimentally the $f_{2}(1270)$ resonance is quite broad $(\Gamma \sim 185$ $\mathrm{MeV}$ ) while in our case, it appears as a bound state (pole in the FRS) of zero width. Contributions as those depicted in Fig. 2 might provide a sizeable width to this pole. Besides there exist other mechanisms like $d$-wave $\pi \pi$ decays, which could also be important in this case because the large available phase space. Those associated to the left diagram of Fig. 2 are considered in Refs. [69, 70]. Regarding our identification of the $f_{2}^{\prime}(1525)$ resonance, we find

11 This latter decay mode can be easily understood from the decay of the resonance to a virtual $\bar{K} K^{*}$ pair. 
dominant couplings to the $K^{*} \bar{K}^{*}$ and $\phi \phi$ channels, which will naturally account for the experimental dominant decay mode into $K \bar{K}$ of this resonance [84] through loop mechanisms (Fig. 21). However, the mass position disagrees much more in this case, while its sizeable coupling in our approach to $\rho \rho$ seems difficult to reconcile with its experimental small branching fractions into $\pi \pi$ and $\pi \pi \pi \pi$. Thus, we have some reservation with this identification, and perhaps it could also be possible to identify the pole with the resonances $f_{2}(1565)$ or $f_{2}(1640)$, which are placed closer to the pole and have decay modes involving an even number of pions, or an $\omega \omega$ pair. Possibly further ingredients, like $d$-wave $K \bar{K}$ pairs, would also be needed to correctly describe the dynamics of the $f_{2}^{\prime}(1525)$ resonance.

In Ref. [70] two states are also generated in this channel, and are associated with the $f_{2}(1270)$ and $f_{2}^{\prime}(1525)$. The real part of both poles agree remarkably well with the masses of these two resonances. This was achieved by a suitable fine-tuning of the subtraction constants. A similar good agreement could not be achieved within our scheme by fine-tuning of the subtraction constants. In [70], these two resonances appear mostly as $\rho \rho$ and $\bar{K}^{*} K^{*}$ bound states, respectively. In our case, these channels are still dominant but with a substantial contribution from the subdominant channels. The hidden gauge interaction for vector mesons model used in [70] and our approach are related for $P V \rightarrow P V$ scattering, thanks to chiral symmetry, but they are completely unrelated in the $V V$ sector, where we believe that the nature of the contact terms can only be unraveled by requiring consistency with the QCD asymptotic behavior at high energies [95]. Besides, the $V V$ interactions of our model are weaker than those deduced in Ref. [70] due to the use of $f_{V}$ instead of the pion decay constant.

TABLE VII: Pole positions and modulus of the couplings in the $(Y, I, J)=(0,1,0)$ sector (MeV units). The subtraction constants $a=-3.5$ for the $P P$ channels and $a=-1$ for the three $V V$ ones have been used. $I^{G}\left(J^{P C}\right)=1^{-}\left(0^{++}\right)$. Possible PDG counterparts: $a_{0}(980), a_{0}(1450)$ and $a_{0}(2020)$.

\begin{tabular}{c|ccccc}
\hline \hline$\sqrt{s_{R}}$ & $\eta \pi$ & $\bar{K} K$ & $\omega \rho$ & $\phi \rho$ & $\bar{K}^{*} K^{*}$ \\
\hline$(991,-46)$ & 2906 & 3831 & 775 & 4185 & 5541 \\
$(1442,-5)$ & 907 & 285 & 10898 & 677 & 3117 \\
$(1760,-12)$ & 790 & 1241 & 667 & 5962 & 5753 \\
\hline \hline
\end{tabular}

\section{Hypercharge 0, isospin 1 and spin 0}

There are five coupled channels in this sector: $\pi \eta, K \bar{K}, \rho \omega, \rho \phi$ and $K^{*} \bar{K}^{*}$ and our model produces three poles in the SRS of the amplitudes. These are compiled in Table VII. The lowest pole should correspond to the $a_{0}(980)$, which has been obtained in all previous studies considering only pseudoscalar-pseudoscalar coupled-channel. In our model, its couplings to the $\pi \eta$ and $K \bar{K}$ are large, in agreement with the results of earlier studies and with the data, but it also presents large couplings to the heaviest channels, $\phi \rho$ and $\bar{K}^{*} K^{*}$.

The pole at $\sqrt{s_{R}}=(1442,-5)$ can be associated to the $a_{0}(1450)$. Within our scheme, it can decay to $\pi \eta$ and $K \bar{K}$, which is in agreement with the data. Its huge coupling to $\omega \rho$ will give rise to a significant $\omega \pi \pi$ decay mode and to an important enhancement of its width, thanks to the broad spectral function of the $\rho$ resonance.

On the other hand, the PDG only reports two $a_{0}$ resonances below $2 \mathrm{GeV}$. Therefore, the third pole in this sector at $\sqrt{s_{R}}=(1760,-12)$ cannot be associated, in principle, to any known state. Nevertheless, it is interesting to note that in Ref. [70] an $a_{0}$-like pole was found located close to the $K^{*} \bar{K}^{*}$ threshold and with large couplings to $K^{*} \bar{K}^{*}$ and $\phi \rho$. On the other hand, a resonance $a_{0}(2020)$ has been reported in [106], with these quantum numbers around $2 \mathrm{GeV}(2025 \pm 30 \mathrm{MeV})$, but extremely wide $(330 \pm 75 \mathrm{MeV})$. The large width of this state makes less meaningful the difference between its mass and that of our pole, which might be then associated to this resonance. Still, it should be noted that the $a_{0}(2020)$ resonance is not yet firmly established and needs further confirmation [84].

\section{E. Hypercharge 0 , isospin 1 and spin 1}

There are two sets of quantum numbers in this sector: $1^{+}\left(1^{+-}\right)$and $1^{-}\left(1^{++}\right)$, corresponding to those of $b_{1}$ and $a_{1}$ resonances. Our results for this channel are compiled in Table VIII]

In the $1^{+}\left(1^{+-}\right)$subsector, two poles are found in the SRS: the lower one can be associated to the $b_{1}(1235)$. The predicted mass, width and decay modes agree well with the data $[\omega \pi$ (dominant), $\rho \eta$ (seen), $4 \pi(<50 \%)$ and 
TABLE VIII: Pole positions and modulus of the couplings $(\mathrm{MeV}$ units $)$ in the $(Y, I, J)=(0,1,1)$ sector. $I^{G}\left(J^{P C}\right)=1^{+}\left(1^{+-}\right)$ $\left[b^{\prime} s\right]$ and $1^{-}\left(1^{++}\right)\left[a^{\prime} s\right]$. A subtraction constant of $a=-1.57$ has been used for positive $G$-parity states. Possible PDG counterparts: $b_{1}(1235), b_{1}(1960), a_{1}(1260), a_{1}(1640)$.

\begin{tabular}{c|cccccccccc|c}
\hline \hline$\sqrt{s_{R}}$ & $\bar{K}^{*} K^{*}$ & $\pi \phi$ & $\pi \omega$ & $\eta \rho$ & $\rho \rho$ & $\left(\bar{K} K^{*}\right)_{S}$ & $\pi \rho$ & $\omega \rho$ & $\left(\bar{K} K^{*}\right)_{A}$ & $\phi \rho$ & $G$ \\
\hline$(1234,-57)$ & 4516 & 438 & 3398 & 900 & 9025 & 3165 & & & & & + \\
$(1642,-139)$ & 10433 & 4214 & 912 & 3321 & 965 & 523 & & & & & + \\
$(1021,-251)$ & & & & & & & 7988 & 7580 & 5284 & 929 & - \\
$(1568,-145)$ & & & & & & & & & & & \\
\end{tabular}

$K \bar{K} \pi(14 \%)]$. This state has also been found in Refs. [37, 43], and this indicates that it mainly originates from the pseudoscalar-vector interaction. The second $b_{1}$ state is found at $\sqrt{s_{R}}=(1642,-139)$. It couples strongly to $K^{*} \bar{K}^{*}$ and lacks a clear PDG counterpart yet. A $b_{1}$ state is also found in Ref. 70] at $\sim 1700 \mathrm{MeV}$. It is tempting to associate our second pole with the resonance $b_{1}(1960)$, though this state is not firmly established yet [106]. The $b_{1}(1960)$ turns out to be also quite wide $(\Gamma=230 \pm 50)$, as it was the case of the $a_{0}(2020)$ resonance above, which makes less important the large difference existing between the masses. Moreover, the data suggest that $b_{1}(1960)$ has non-zero overlaps with the $\pi \omega$ and $\eta \pi \omega$ channels [107]. This is compatible with the features of our pole. (Note that the $\eta \rho$ coupling could lead to a non zero contribution to the $\eta \pi \omega$ decay mode.)

In the $1^{-}\left(1^{++}\right)$subsector, also two poles are found in the SRS. It is tempting to associate them to the $a_{1}(1260)$ and $a_{1}(1640)$, the only two $a_{1}$ resonances below $2 \mathrm{GeV}$ reported in the PDG [84].

The mass and width of $a_{1}$ (1260) suffer from large uncertainties, being quoted in the PDG values of $1230 \pm 40 \mathrm{MeV}$ and $250-600 \mathrm{MeV}$, respectively. Its dominant decay modes are $3 \pi$ and $\left(\bar{K} K^{*}\right)_{A}$. This is in total agreement with the largest couplings of our lightest pole in this sector. In addition, the main properties of this pole are similar to those of the pole found in the approach of Ref. [43].

The resonance $a_{1}(1640)$ is much worse established experimentally and it is not reported in the approach of Ref. [70]. Nevertheless, our second pole couples strongly to $V V$ channels and its features fit well with those known for the $a_{1}(1640)$ resonance.

TABLE IX: Pole positions and modulus of the couplings (MeV units ) in the $(Y, I, J)=(0,1,2)$ sector. $I^{G}\left(J^{P C}\right)=1^{-}\left(2^{++}\right)$. The subtraction constant has been set to $a=-3.4$. Possible PDG counterparts: $a_{2}(1320)$ and $a_{2}(1700)$.

\begin{tabular}{c|ccc}
\hline \hline$\sqrt{s_{R}}$ & $\omega \rho$ & $\phi \rho$ & $\bar{K}^{*} K^{*}$ \\
\hline$(1228,0)$ & 11287 & 2637 & 6281 \\
$(1775,-6)$ & 1454 & 3167 & 4362 \\
\hline \hline
\end{tabular}

\section{F. Hypercharge 0, isospin 1 and spin 2}

There are three coupled channels in this sector: $\bar{K}^{*} K^{*}, \omega \rho$, and $\phi \rho$, and we find two poles, one in the FRS and a second one in the SRS of the amplitudes (see Table IX), which might be associated to the $a_{2}(1320)$ and $a_{2}(1700)$ resonances. In our model, the bound state strongly couples to the $\omega \rho$ channel, which would give rise to the observed $3 \pi$ and $\omega \pi \pi$ decay modes of the $a_{2}(1320)$ thanks to the width of the virtual $\rho$ meson. Furthermore, if the pole position were closer to the experimental mass, the width would also increase. Fine-tuning of the subtraction constant did not work to achieve a better agreement in the mass position.

Little is known about the $a_{2}(1700)$, but the assignment of our second pole with it might get supported by the decays of this resonance into $\omega \rho$ and $K \bar{K}$ pairs. Indeed, this latter decay mode can be obtained from the decays of the resonance to virtual $\phi \rho$ or $K^{*} \bar{K}^{*}$ pairs, through loop mechanisms as those depicted in Fig. 2, The hidden gauge interaction for vector mesons model used in [70] gives rise only to one pole, whose features corresponds to the 
heaviest of the poles found here. As it is the case here, though its mass is close to that quoted in the PDG for the $a_{2}(1700)$ resonance, it turns out to be much narrower than this resonance. This could be an indication of the fact that either the identification of this pole with the $a_{2}(1700)$ resonance is incorrect or that other mechanisms, such as coupled-channel $d$-wave dynamics, might play an important role in this case.

TABLE X: Pole positions and modulus of the couplings $\left(\mathrm{MeV}\right.$ units) in the $(Y, I, J)=(1,1 / 2,0)$ sector. $I\left(J^{P}\right)=\frac{1}{2}\left(0^{+}\right)$. The subtraction constant has been set to $a=-1.5$ for the $V V$ channels. Possible PDG counterparts: $K_{0}^{*}(800), K_{0}^{*}(1430)$ and $K_{0}^{*}(1950)$.

\begin{tabular}{c|ccccc}
\hline \hline$\sqrt{s_{R}}$ & $K \pi$ & $\eta K$ & $K^{*} \rho$ & $K^{*} \omega$ & $K^{*} \phi$ \\
\hline$(830,-170)$ & 4446 & 1879 & 5868 & 2029 & 1805 \\
$(1428,-24)$ & 1805 & 892 & 8007 & 10803 & 5556 \\
$(1787,-37)$ & 45 & 2662 & 657 & 1107 & 12181 \\
\hline \hline
\end{tabular}

\section{G. Hypercharge 1, isospin 1/2 and spin 0}

In this sector there are five coupled channels: $\pi K, \eta K, \rho K^{*}, \omega K^{*}$, and $\phi K^{*}$, and three poles are found in the SRS of the amplitudes. The first one at $\sqrt{s_{R}}=(830,-170)$ can be associated to the $K_{0}^{*}(800)$. There is still a controversy about the existence and the origin [108] of this broad resonance $(\Gamma \sim 550 \mathrm{MeV})$, being $K \pi$ its dominant decay mode. It is very similar to the $f_{0}(600)$, and hence it cannot be interpreted as a Breit-Wigner narrow resonance.

We identify the second pole at $\sqrt{s_{R}}=(1428,-24)$ with the $K_{0}^{*}(1430)$ resonance, despite being the latter one much wider than the pole found in our scheme. The $K \pi$ branching fraction for this resonance is $93 \% \pm 10 \%[84]$. The pole generated in our scheme couples more than twice stronger to the $K \pi$ channel than to the $\eta K$ one, which is also open. However, the coupling to the $K^{*} \rho$ channel is four times bigger and does not contribute to the width of $48 \mathrm{MeV}$ quoted in Table $\mathrm{X}$ because it is not open. Nevertheless, the resonance can decay into a virtual $K^{*} \rho$ pair which will significantly enhance the $K \pi$ decay probability, thanks to the broad $\rho$ and $K^{*}$ widths and the fact that the pole is not placed too far from threshold (see left panel of Fig. 2).

In Ref. [70], where only $V V$-channels are considered, only one pole at $(1643,-24)$ with a strong $\rho K^{*}$ coupling was found. The authors of [70] argue, although with reservations, that it might correspond to the $K(1630)$ resonance. We conjecture that with an adequate subtraction constant the pole found in that reference might be similar to our second pole and thus it would rather correspond to the $K_{0}^{*}(1430)$ resonance.

The situation of the third pole is less clear. It would be tempting to associate this third pole at $\sqrt{s_{R}}=(1787,-37)$ to the $K(1630)$ (with yet undetermined $J^{P}[84]$ ). Our pole is wider than the $K(1630)$ resonance, whose reported width is compatible with zero. This could be explained because our pole is located above the $K^{*} \omega$ threshold and experimentally it is below this threshold although close to it. Note that this channel gives rise to a decay mode $K \pi \pi$, as reported in the PDG. Nevertheless, we believe that such identification would probably be incorrect, since the biggest couplings of the pole found here are those corresponding to the $\eta K$ and $K^{*} \phi$ channels. The first of these two channels is open, giving rise to a sizeable width difficult to reconcile with the narrow width quoted in the PDG for the $K(1630)$. Besides, the huge $K^{*} \phi$ coupling will lead to a $K \pi$ decay mode, through the loop mechanisms sketched in Fig. 2 while the decay mode observed in the PDG is $K \pi \pi$. Note that the pole at $\sqrt{s_{R}}=(1787,-37)$ also couples to the $K^{*} \rho$ channel and that it will also contribute to the $K \pi$ decay mode. This suggests to identify the pole found here with the wide $K_{0}^{*}(1950)$ resonance, for which the decay mode observed in the PDG is $K \pi$. Moreover, its large width $201 \pm 90 \mathrm{MeV}$ [84]) make less meaningful the difference between its mass $1945 \pm 22$ and that of our pole. However, it should be pointed out that the $K_{0}^{*}(1950)$ resonance is not firmly established yet and needs further confirmation [84].

\section{H. Hypercharge 1, isospin $1 / 2$ and spin 1}

In this sector there are eight coupled channels: $K^{*} \pi, K \rho, K \omega, K^{*} \eta, K \phi, K^{*} \rho, K^{*} \omega, K^{*} \phi$, and four poles are found on the SRS.

In the PDG there appear three resonances below $2 \mathrm{GeV}$ with these quantum numbers, namely $K_{1}(1270), K_{1}(1400)$, $K_{1}(1650)$, while here we found four poles. 
TABLE XI: Pole positions and modulus of the couplings (MeV units) in the $(Y, I, J)=(1,1 / 2,1)$ sector. $I\left(J^{P}\right)=\frac{1}{2}\left(1^{+}\right)$. The subtraction constants has been set to $a=-2.5$ for the $P V$ channels and to $a=-1.7$ for the three $V V$ ones. Possible PDG counterparts: $K_{1}(1270), K_{1}(\ldots), K_{1}(1400), K_{1}(1650)$.

\begin{tabular}{c|ccccccccc}
\hline \hline$\sqrt{s_{R}}$ & $\pi K^{*}$ & $K \rho$ & $K \omega$ & $\eta K^{*}$ & $K \phi$ & $K^{*} \rho$ & $K^{*} \omega$ & $K^{*} \phi$ \\
\hline$(1188,-64)$ & 5616 & 3703 & 1959 & 1988 & 1860 & 4405 & 2824 & 2669 \\
$(1250,-31)$ & 3910 & 5267 & 2516 & 3612 & 1665 & 3225 & 6311 & 2023 \\
$(1414,-66)$ & 798 & 3326 & 3030 & 1169 & 1668 & 9866 & 2225 & 4373 \\
$(1665,-95)$ & 1358 & 1166 & 922 & 3650 & 3799 & 2880 & 3017 & 10641 \\
\hline \hline
\end{tabular}

In Ref. [43] two poles $\left(\sqrt{s_{R}}=(1112,-64) \mathrm{MeV}\right.$ and $\left.\sqrt{s_{R}}=(1216,-4) \mathrm{MeV}\right)$ were reported, using only $P V \rightarrow P V$ interaction. An additional pole was found at $\sqrt{s_{R}}=(1737,-82) \mathrm{MeV}$ in Ref. [70], using only the $V V \rightarrow V V$ sector. The work of Roca et al. [43] was revisited in Ref. [109]. In this latter reference, the double pole structure of the $K_{1}(1270)$, uncovered in [43], is further confirmed. Let us summarize here some of the most relevant findings of Ref. [109]. There, one pole is found at $\sim 1200 \mathrm{MeV}$ with a width of $\sim 250 \mathrm{MeV}$ and the other is found at $\sim 1280 \mathrm{MeV}$ with a width of $\sim 150 \mathrm{MeV}$. The lower pole couples more to the $K^{*} \pi$ channel and the higher pole couples dominantly to the $K \rho$ channel. The peak in the $K \pi \pi$ mass distribution in the WA3 data [110] on $K^{-} p \rightarrow K^{-} \pi^{+} \pi^{-} p$ is explained in [109] as a superposition of two poles, but in the $K^{*} \pi$ channel the lower pole dominates and in the $\rho K$ channel, the higher pole gives the biggest contribution. Finally, it is argued in [109] that different reaction mechanisms may prefer different channels and this would explain the different invariant mass distributions seen in various experiments.

The results compiled in Table XI, show two poles around 1.2 GeV which correspond to those reported in Ref. [109], though the couplings turn out to be somehow different. This is partially due to the inclusion here of the $V V$ channels. When those channels are switched off, the agreement improves, but there still remain some differences between the couplings obtained in both approaches, specially on the strength of the $K^{*} \eta$ coupling for the lightest resonance. This can be attributed, at this stage, to the approximation $m_{P}=0$ used here when computing the potential. Our scheme implements an extra SU(6) symmetry breaking pattern induced by the use of different pseudoscalar and vector decay constants $\left(f_{P} \neq f_{V}\right)$, however, in Ref. [109] the WA3 $K^{-} p \rightarrow K^{-} \pi^{+} \pi^{-} p$ data were successfully fitted with a value of $f^{2} \sim(115 \mathrm{MeV})^{2}$, which numerically is rather similar to $f_{P} f_{V}$ used here (see Table II). On the other hand, taking into account the finite $\rho$ and $K^{*}$ widths in the intermediate loops will increase the imaginary parts of the poles, specially that of the higher pole which has a large coupling to the $\rho K$ channel. This will then bring its width close to $\sim 150 \mathrm{MeV}$, as found in in Ref. [109]. Thus, our findings here reinforce the double pole picture for the $K_{1}(1270)$ resonance predicted in Refs. [43, 109]. It is also noteworthy that Ref. [37] did not find this double pole structure.

We move now to the third of the poles found here $\left[\sqrt{s_{R}}=(1414,-66) \mathrm{MeV}\right]$, which has large $K^{*} \pi, K \rho, K \omega, K^{*} \phi$ and specially $K^{*} \rho$ couplings. Given its mass and width, it can be naturally associated to the $K_{1}(1400)$ resonance. However in the PDG, branching fractions of $(94 \pm 6) \%,(3 \pm 3) \%$ and $(1 \pm 1) \%$, for the $K^{*} \pi, K \rho$ and $K \omega$ modes, respectively, are quoted for this resonance. The couplings shown in Table XI cannot be easily reconciled with the above fractions. Refs. [43, 109] did not find the $K_{1}(1400)$ resonance, while in Ref. [37] a broad bump in the speed plot was associated to it. In the $V V$-work of Ref. [70], a pole at $\sqrt{s_{R}}=(1737,-82) \mathrm{MeV}$ is reported with a dominant $\rho K^{*}$ coupling. Indeed, when the $P V-V V$ interferences are switched off, we find the two $K_{1}(1270)$ poles in the $P V$ sector and a third pole in the $V V$ sector with a large $\rho K^{*}$ coupling, whose position depends strongly on the value of the subtraction constant. Our conjecture is that it is precisely this pole, which manifests itself as a $\rho K^{*}$ bound or resonant state when only $V V$ interactions are considered, the one that moves down to $\sqrt{s_{R}}=(1414,-66)$ when the $P V$ channels are also included.

Here, we envisage two different possibilities:

(i) To identify the $\sqrt{s_{R}}=(1414,-66)$ pole with the $K_{1}(1400)$ resonance, despite the PDG branching fractions quoted above. It is worth stressing here that the properties quoted in the PDG obtained from the WA3 data analysis rely upon considering only one pole for the $K_{1}(1270)$. The reanalysis of the WA3 data carried out in [109], where the double $K_{1}(1270)$ pole structure is taken into account and the totality of the $P V$ channels studied here are considered, is also inconsistent with the PDG $K_{1}(1400)$ branching fractions, being the $K \rho$ mode almost comparable to the $K^{*} \pi$ one (see Fig. 7 of this reference) and certainly it is not around 30 times smaller. On the other hand, there exists another ingredient which should be considered. Our state has a huge $K^{*} \rho$ coupling, which will provide a $K \pi \pi \pi$ signature and that of course will also contribute to the inclusive WA3 $K^{-} p \rightarrow K^{-} \pi^{+} \pi^{-} p$ reaction. This latter mechanism was considered neither in the original analysis of Ref. [110] 
nor in the better theoretical founded re-analysis of Ref. [109].

Within this scenario, the fourth pole at $\sqrt{s_{R}}=(1665,-95)$ shown in Table XI could be assigned to the $K_{1}(1650)$ with a mass of $1650 \pm 50 \mathrm{MeV}$ and a width of $150 \pm 50$ [84]. The only decay channels observed are $K \pi \pi$ and $K \phi$, which could be easily associated to the large $K \phi$ coupling of the pole together with its sizeable $K^{*} \pi$ and $K \rho$ components (see Table XI). This pole appears due to the interplay between the $K^{*} \phi$ and $K \phi$ channels, similarly as it was discussed earlier in the case of the $h_{1}(1595)$, and indeed it disappears when only the $V V$ sector is considered. In Ref. [70], the above mentioned $\sqrt{s_{R}}=(1737,-82)$ pole was tentatively assigned to the $K_{1}(1650)$ resonance despite the fact that its large $\rho K^{*}$ coupling is difficult to accommodate with the $K_{1}(1650)$ known decays.

Nevertheless, this is still a questionable scenario, since the couplings quoted in Table XI, for the pole at $(1414,-66)$, indicate that its $K \rho$ decay mode is much larger than the $K^{*} \pi$, and this is difficult to reconcile even with the results of the re-analysis of Ref. [109].

(ii) Alternatively, the subtraction constants could be fine-tuned so that the third pole is pushed up in energy and thus it could be associated to the $K_{1}(1650)$ (see for instance Table XII). Properties of the poles, other than the mass and width, are not much affected by the fine-tuning, and one still gets the two pole structure for the $K_{1}(1270)$ resonance. In this scenario no pole is assigned to the $K_{1}(1400)$, which then will not be dynamically generated, as advocated in the picture of Refs. [43, 70, 109]. However, the assignment of the third pole to the $K_{1}(1650)$ would suffer from the problems mentioned above in the case of the $\sqrt{s_{R}}=(1737,-82)$ resonance found in Ref. [70]. In addition, a further $K_{1}$ above $1.8 \mathrm{GeV}$ and not included in the PDG, will be predicted with a large $K \phi$ decay mode.

TABLE XII: Same as Table XI $\left[I\left(J^{P}\right)=\frac{1}{2}\left(1^{+}\right)\right]$, but using subtraction constants $a=-3.1$ for the $P V$ channels and $a=-1.0$ for the three $V V$ ones. Possible PDG counterparts: $K_{1}(1270), K_{1}(\ldots), K_{1}(1650), K_{1}(\ldots)$.

\begin{tabular}{c|ccccccccc}
\hline \hline$\sqrt{s_{R}}$ & $\pi K^{*}$ & $K \rho$ & $K \omega$ & $\eta K^{*}$ & $K \phi$ & $K^{*} \rho$ & $K^{*} \omega$ & $K^{*} \phi$ \\
\hline$(1169,-46)$ & 4595 & 3643 & 1862 & 1873 & 1484 & 2468 & 2328 & 1418 \\
$(1266,-44)$ & 4539 & 5789 & 2797 & 4504 & 2870 & 1077 & 7956 & 1837 \\
$(1576,-43)$ & 888 & 1868 & 2508 & 796 & 682 & 9874 & 1364 & 3366 \\
$(1823,-61)$ & 474 & 154 & 221 & 2636 & 3105 & 770 & 1295 & 11756 \\
\hline \hline
\end{tabular}

TABLE XIII: Pole positions and modulus of the couplings $(\mathrm{MeV}$ units $)$ in the $(Y, I, J)=(1,1 / 2,2)$ sector. $I\left(J^{P}\right)=\frac{1}{2}\left(2^{+}\right)$. Possible PDG counterparts: $K_{2}^{*}(1430)$.

\begin{tabular}{c|ccc}
\hline \hline$\sqrt{s_{R}}$ & $K^{*} \rho$ & $K^{*} \omega$ & $K^{*} \phi$ \\
\hline$(1708,-156)$ & 7227 & 2834 & 2299 \\
\hline \hline
\end{tabular}

\section{Hypercharge 1 , isospin $1 / 2$ and spin 2}

In this sector, a pole is found in the SRS of the amplitudes. In the PDG, two $K_{2}^{*}$ resonances below 2 GeV $\left[K_{2}^{*}(1430)\right.$ and $\left.K_{2}^{*}(1980)\right]$ are reported, though only the lightest one is firmly established. The $K_{2}^{*}(1430)$ has a mass of $1429 \pm 1.4 \mathrm{MeV}$ and a width of $104 \pm 4 \mathrm{MeV}$; the second resonance has a mass of $1973 \pm 26 \mathrm{MeV}$ and a width of $373 \pm 70 \mathrm{MeV}$. It is not clear to which one to associate the state we find. The subtraction constants cannot be fine-tuned to achieve the mass of the pole to lie much closer to $1.43 \mathrm{GeV}$ than in Table XIII. Nevertheless, we believe that the pole found here might correspond to the $K_{2}^{*}(1430)$ and its nature is somehow related to those of the $f_{2}(1270)$ and $f_{2}^{\prime}(1525)$. In both cases, an important influence of $d$-wave interactions is to be expected. Indeed in the case of the $K_{2}^{*}(1430)$, the PDG branching fractions are around $50 \%, 25 \%, 9 \%$ and $3 \%$ for the $d$-wave modes $K \pi, K^{*} \pi, K \rho$ 
and $K \omega$, respectively. In addition, the branching fraction of the $K^{*} \pi \pi$ channel is only about $13 \%$. This latter decay mode looks like the only one more or less related to the dynamics included within our model, thanks to the dominant coupling $K^{*} \rho$ of the pole displayed in Table XIII. This would explain why our model does not describe properly the mass and the width of the $K_{2}^{*}(1430)$. From this point of view, what is somewhat more surprising is the fact that our scheme were able to describe the mass of the $f_{2}(1270)$ at all. However, there is here a distinctive feature: the possible influence of the $d$-wave pseudoscalar-vector meson $K^{*} \pi$ channel, which lies closer to the resonance mass than the pseudoscalar-pseudoscalar channels. Notice that the equivalent channel in the case of $f_{2}(1270)$ would be $\pi \rho$, but it is not allowed by $G$-parity conservation.

The approach of Ref. 70] for $V V \rightarrow V V$ scattering produces a resonance in this sector, with mass fine-tuned to $1430 \mathrm{MeV}$, even if all type $d$-wave interactions are also ignored.

\section{J. Exotics}

Exotics refers here to meson states with quantum numbers that cannot be formed by a $q \bar{q}$ pair. Quantum numbers with $I \geq 3 / 2$ or $|Y|=2$ are exotic. Our model produces five poles on the complex plane with the following quantum numbers: $2^{+}\left(0^{++}\right)$with $Y=0,3 / 2\left(0^{+}\right)$and $3 / 2\left(1^{+}\right)$with $Y=1$, and $0\left(1^{+}\right)$and $1\left(0^{+}\right)$with $Y=2$. Remarkably, no exotic state was reported in Ref. 70]. This is a direct consequence of the different dynamics implicit in both approaches. Future experiments may be needed to distinguish between these two schemes.

TABLE XIV: Poles positions and modulus of the couplings (MeV units) in the $(Y, I, J)=(0,2,0)$ sector. The subtraction constant has been set to $a=-1.5 . I^{G}\left(J^{P C}\right)=2^{+}\left(0^{++}\right)$. Possible PDG counterparts: $X(1420)$.

\begin{tabular}{c|cc}
\hline \hline$\sqrt{s_{R}}$ & $\pi \pi$ & $\rho \rho$ \\
\hline$(1419,-54)$ & 2719 & 10069 \\
\hline \hline
\end{tabular}

\section{Hypercharge 0, isospin 2 and spin 0}

In this sector, a pole is found that, given its mass and width, can be naturally associated to the $X(1420)$ resonance (see Table XIV]). This resonance needs further confirmation and its current evidence comes from a statistical indication 111 for a $\pi^{+} \pi^{+}$resonant state in the $\bar{n} p \rightarrow \pi^{+} \pi^{+} \pi^{-}$annihilation reaction with data collected by the OBELIX experiment. Within our scheme, the pole is essentially a $\rho \rho$ bound state with a small coupling to the $\pi \pi$ channel that moves the pole to the SRS. The fact that the strength of the coupling to $\pi^{+} \pi^{+}$is not large might explain why the resonance distorts weakly the spectrum of the outgoing pair of positive pions in the OBELIX data. Within our scheme, the $\rho \rho \rightarrow \rho \rho$ amplitude is symmetric under $I \leftrightarrow J$ exchange. For $\mathcal{D}_{\text {kin }}$ this comes as a result of $\mathrm{SU}(6)$ symmetry. On the other hand, the interaction $\mathcal{D}_{m}$ is a contact term and this ensures the invariance under $I \leftrightarrow J .{ }^{12}$ As a consequence our $\rho \rho$ potential in this sector $(I=2, J=0)$ is the same as that in the $I=0, J=2$ one. BS amplitudes in both sectors will become different because of coupled-channel and renormalization effects. Nevertheless, we expect the $X(1420)$ to be the counterpart of the $f_{2}(1270)$, which appeared mostly as a $\rho \rho J=2$ isoscalar bound state. This situation is distinctively different in the hidden gauge interaction model used of Ref. [70], where near threshold, the $\rho \rho$ interaction in the $I=2, J=0$ sector becomes repulsive and five times smaller, in absolute value, than that in the $I=0, J=2$ sector [69]. Indeed, while in the latter sector the $\rho \rho$ interaction is attractive and gives rise to the $f_{2}(1270)$ resonance, the model of Ref. [69, 70] does not provide any $(I=2, J=0)$ resonance. However, it is found a dip in the $\rho \rho$ amplitude squared in this latter work. There, it is suggested that such a dip in the $\rho \rho$ amplitude might lead to a bump in $\pi^{+} \pi^{+}$production.

The $\pi \pi$ diagonal potential is repulsive in this sector, however, the $\pi \pi \rightarrow \rho \rho$ transition potential leads to an interaction more attractive than that deduced from the diagonal $\rho \rho$ potential. Indeed, from Tables XXVII and XLVI, one finds eigenvalues \pm 2 for $\mathcal{D}_{\text {kin }}$ and $8 / 3,-8$ for $\mathcal{D}_{m}$ (the two matrices entering in the kernel potential) while the

12 Indeed, the most general contact interaction in the $\rho \rho$ sector is of the form $\mathcal{L}_{\text {int }}=g_{1} \rho_{a i} \rho_{a i} \rho_{b j} \rho_{b j}+g_{2} \rho_{a i} \rho_{a j} \rho_{b i} \rho_{b j}$ which is symmetric under exchange of spin and isospin labels. 
$\rho \rho$ diagonal matrix elements are -1 and $-16 / 3$, respectively. [Notice that our conventions are such that negative diagonal matrix elements, or eigenvalues, of $\mathcal{D}_{\text {kin }}, \mathcal{D}_{m}$ and $\mathcal{D}_{a}$ correspond to attractive interactions.]

TABLE XV: Pole positions and modulus of the couplings (MeV units) in the $(Y, I, J)=(1,3 / 2,0)$ sector. $I^{G}\left(J^{P}\right)=\frac{3}{2}\left(0^{+}\right)$.

\begin{tabular}{c|cc}
\hline \hline$\sqrt{s_{R}}$ & $K \pi$ & $K^{*} \rho$ \\
\hline$(1433,-70)$ & 3242 & 10962 \\
\hline \hline
\end{tabular}

TABLE XVI: Pole positions and modulus of the couplings $(\mathrm{MeV}$ units $)$ in the $(Y, I, J)=(2,1,0)$ sector. $I\left(J^{P}\right)=1\left(0^{+}\right)$.

\begin{tabular}{c|cc}
\hline \hline$\sqrt{s_{R}}$ & $K K$ & $K^{*} K^{*}$ \\
\hline$(1564,-66)$ & 3484 & 11593 \\
\hline \hline
\end{tabular}

\section{Hypercharge 1, isospin 3/2 and spin 0 and hypercharge 2, isospin 1 and spin 0}

The matrices $\mathcal{D}_{\text {kin }}$ and $\mathcal{D}_{m}$ are the same in both sectors, and identical to those appearing in $(Y, I, J)=(0,2,0)$. Thus, the two resonances displayed in Tables XV] and XVI belong to the same multiplet of scalars that the resonance $X(1420)$, and masses and widths are similar. We will come back to this point below.

TABLE XVII: Pole positions and modulus of the couplings $(\mathrm{MeV}$ units $)$ in the $(Y, I, J)=(1,3 / 2,1)$ sector. $I\left(J^{P}\right)=\frac{3}{2}\left(1^{+}\right)$.

\begin{tabular}{c|ccc}
\hline \hline$\sqrt{s_{R}}$ & $\pi K^{*}$ & $K \rho$ & $K^{*} \rho$ \\
\hline$(1499,-127)$ & 3791 & 3699 & 8513 \\
\hline \hline
\end{tabular}

3. Hypercharge 1, isospin 3/2 and spin 1 and hypercharge 2, isospin 0 and spin 1

We find one pole in each sector (see Tables XVII and XVIII). Masses and widths of these two resonances are quite similar and we will argue below that they belong to the same axial vector multiplet.

4. Hypercharge 0, isospin 2 and spin 1 and 2, hypercharge 1, isospin 3/2 and spin 2, and hypercharge 2, isospin 1 and spin 1 and 2

The interaction in these five sectors is repulsive and they present no poles.

\section{SUMMARY AND CONCLUSIONS}

Tables XIX and XX compile the different poles found within the present approach. It must be observed that the widths obtained are only a first approximation and they could receive substantial corrections in some cases. This is because of the following reasons. First, the only decay channels considered are $P P, P V$ and $V V s$-wave pairs. Second, the widths of the vector mesons have been neglected in their propagators in the loop functions. The effect might be particularly important for the $\rho$ and the $K^{*}$ resonances. It is to be expected that this mechanism will enhance the width of the resonances with a very small impact on the masses [43]. The same mechanism should also introduce 
TABLE XVIII: Pole positions and modulus of the couplings $\left(\mathrm{MeV}\right.$ units) in the $(Y, I, J)=(2,0,1)$ sector. $I\left(J^{P}\right)=0\left(1^{+}\right)$.

\begin{tabular}{c|cc}
\hline \hline$\sqrt{s_{R}}$ & $K K^{*}$ & $K^{*} K^{*}$ \\
\hline$(1608,-114)$ & 5614 & 9303 \\
\hline \hline
\end{tabular}

contributions of the type displayed in Fig. 2 Such contributions have appeared repeatedly during the discussion of our results.

SU(6) symmetry of our approach has been explicitly broken to account for physical masses and decay constants, and also when the amplitudes have been renormalized. Nevertheless, the underlying SU(6) symmetry is still present and serves to organize the set of even parity meson resonances found in this work, and compiled in Tables XIX] and $\mathrm{XX}$

Spin-flavor symmetry has been used to guide the construction of the $s$-wave interactions among the members of the $\mathrm{SU}(6) 35$ multiplet. The matrix $\mathcal{D}_{\text {kin }}$ that appears in the kinetic term of the amplitudes can be expressed as

$$
\mathcal{D}_{\text {kin }}=-12 P_{\mathbf{1}}-6 P_{\mathbf{3 5}}-2 P_{\mathbf{1 8 9}}+2 P_{\mathbf{4 0 5}} .
$$

Therefore, this interaction is (moderately) repulsive in the $\mathbf{4 0 5}$ representation and attractive in the other representations. To the extent that $\mathcal{D}_{\text {kin }}$ is the dominant term, this favors the existence of up to $225(1+35+189)$ states. (This counts all states of spin and isospin as different, not only multiplets. In terms of $J^{P} I^{G} Y$ states, this number is 45 .) The irreducible representations (irreps) of $\mathrm{SU}(6)$ can be reduced in terms of irreps of $\mathrm{SU}(3) \otimes \mathrm{SU}(2)$. In this way, the content of the $\mathrm{SU}(6) \mathbf{1}, \mathbf{3 5}_{s}, \mathbf{1 8 9}$ irreps is as follows

$$
\begin{aligned}
\mathbf{1} & =1_{1}, \\
\mathbf{3 5} & =8_{1} \oplus 8_{3} \oplus 1_{3}, \\
\mathbf{1 8 9} & =27_{1} \oplus 8_{1} \oplus 1_{1} \oplus 10_{3} \oplus 10_{3}^{*} \oplus 8_{3} \oplus 8_{3} \oplus 8_{5} \oplus 1_{5},
\end{aligned}
$$

where the subindex refers to $2 J+1$, so e.g., $10_{3}^{*}$ stands for the representation $10^{*}$ of $\mathrm{SU}(3)$ with $J=1$. Further, the $(Y, I)$ content of the $\mathrm{SU}(3)$ irreps is as follows

$$
\begin{aligned}
1 & =(0,0), \\
8 & =( \pm 1,1 / 2),(0,1),(0,0), \\
10 \oplus 10^{*} & =( \pm 1,3 / 2),(0,1),(0,1),( \pm 1,1 / 2),( \pm 2,0), \\
27 & =( \pm 2,1),( \pm 1,3 / 2),( \pm 1,1 / 2),(0,2),(0,1),(0,0) .
\end{aligned}
$$

The gross features of the states reported in Tables $\mathrm{XIX}$ and $\mathrm{XX}$ follow the above decomposition based on SU(6) multiplets. This picture is somewhat modified by the effect of the terms added to the kinetic contribution of the Hamiltonian (see Eq. (40)), namely, $\mathcal{D}_{m}$, which is mainly attractive and $\mathcal{D}_{a}$, which is repulsive. As mentioned, the use of different vector and pseudoscalar meson masses and decay constants, and the used subtraction constants, which in some cases have been fine-tuned to better reproduce the experimental (PDG) resonances, produce also a deviation from the $\mathrm{SU}(6)$ pattern.

In Table I] the poles found in this work (Tables $\mathrm{XIX}$ and $\mathrm{XX}$ ) are classified in terms of the above SU(6) and $\mathrm{SU}(3) \otimes \mathrm{SU}(2)$ irreps. Several comments are in order here. First, it should be stressed that there will be mixings between states with the same $J^{P} I^{G} Y$ quantum numbers but belonging to different $\mathrm{SU}(6)$ and/or $\mathrm{SU}(3)$ multiplets, since these symmetries are broken both within our approach and in nature. These mixings have not been considered when classifying the states in Table I. Some comments are also pertinent regarding each spin-parity sector:

(i) $J^{P}=0^{+}$: As can be seen in Table I the poles found here closely follow the pattern determined by the spinflavor SU(6) symmetry, except for the absence of the singlet state associated to the $\mathbf{1 8 9} \mathrm{SU}(6)$ irrep. The attractive interaction in this irrep is weak. We have checked that if the SU(6) symmetry breaking contact term $\left(\mathcal{D}_{m}\right)$ is switched off a new $f_{0}$ resonance (with a mass close to $1.9 \mathrm{GeV}$ ) would be generated in our calculation, corresponding to this $1_{1}$ missing state. On the other hand, the $\mathrm{SU}(6)$ pattern is also accurate when describing the PDG scalar resonances compiled in Table【 This fact has two consequences. First, it increases the credibility of our predictions on the existence of two exotic states in the region 1.4-1.6 GeV, belonging to the SU(3) 27 irrep included in the SU(6) 189, while giving further theoretical support on the reliability of other resonances, not yet firmly established, as for example the $a_{0}(2020)$ or the exotic isotensor $X(1420)$ state. Second, by inspection 
TABLE XIX: Poles having non exotic quantum numbers found in this work and possible PDG counterparts. Units are given in $\mathrm{MeV}$. Those resonances marked with $\dagger$ need to be confirmed. A question mark symbol expresses our reservations on the assignment.

\begin{tabular}{|c|c|c|c|c|c|}
\hline \multirow[t]{2}{*}{$(Y, I, J)$} & \multirow[t]{2}{*}{$I^{G}\left(J^{P C}\right)$} & \multirow{2}{*}{$\begin{array}{c}\text { This model } \\
\text { Pole position }\left(\sqrt{s_{R}}\right)\end{array}$} & \multicolumn{3}{|c|}{ PDG [84] } \\
\hline & & & Name & Mass & Width \\
\hline \multirow[t]{4}{*}{$(0,0,0)$} & $0^{+}\left(0^{++}\right)$ & $(635,-202)$ & $f_{0}(600)$ & $400 \sim 1200$ & $600 \sim 1000$ \\
\hline & $0^{+}\left(0^{++}\right)$ & $(969,0)$ & $f_{0}(980)$ & $980 \pm 10$ & $40 \sim 100$ \\
\hline & $0^{+}\left(0^{++}\right)$ & $(1350,-62)$ & $f_{0}(1370)$ & $1200 \sim 1500$ & $200 \sim 500$ \\
\hline & $0^{+}\left(0^{++}\right)$ & $(1723,-52)$ & $f_{0}(1710)$ & $1720 \pm 6$ & $135 \pm 8$ \\
\hline \multirow[t]{4}{*}{$(0,0,1)$} & $0^{-}\left(1^{+-}\right)$ & $(1006,-85)$ & $h_{1}(1170)$ & $1170 \pm 20$ & $360 \pm 40$ \\
\hline & $0^{-}\left(1^{+-}\right)$ & $(1373,-17)$ & $h_{1}(1380)^{\dagger}$ & $1386 \pm 19$ & $91 \pm 30$ \\
\hline & $0^{-}\left(1^{+-}\right)$ & $(1600,-67)$ & $h_{1}(1595)^{\dagger}$ & $1594_{-60}^{+18}$ & $384_{-120}^{+90}$ \\
\hline & $0^{+}\left(1^{++}\right)$ & $(1286,0)$ & $f_{1}(1285)$ & $1281.8 \pm 0.8$ & $24.3 \pm 1.1$ \\
\hline \multirow[t]{3}{*}{$(0,0,2)$} & $0^{+}\left(2^{++}\right)$ & $(1289,0)$ & $f_{2}(1270)$ & $1275.1 \pm 1.2$ & $185.1_{-2.4}^{+2.9}$ \\
\hline & $0^{+}\left(2^{++}\right)$ & $(1783,-19)$ & $f_{2}(1640)^{\dagger}$ or $f_{2}^{\prime}(1525)$ & $1639 \pm 6$ & $99_{-40}^{+60}$ \\
\hline & & & or $f_{2}(1430)^{\dagger}$ or $f_{2}(1565)^{\dagger}, \ldots$ & & \\
\hline \multirow[t]{3}{*}{$(0,1,0)$} & $1^{-}\left(0^{++}\right)$ & $(991,-46)$ & $a_{0}(980)$ & $980 \pm 20$ & $50 \sim 100$ \\
\hline & $1^{-}\left(0^{++}\right)$ & $(1442,-5)$ & $a_{0}(1450)$ & $1474 \pm 19$ & $265 \pm 13$ \\
\hline & $1^{-}\left(0^{++}\right)$ & $(1760,-12)$ & $a_{0}(2020)^{\dagger} ?$ & $2025 \pm 30$ & $330 \pm 75$ \\
\hline \multirow[t]{4}{*}{$(0,1,1)$} & $1^{+}\left(1^{+-}\right)$ & $(1234,-57)$ & $b_{1}(1235)$ & $1229.5 \pm 3.2$ & $142 \pm 9$ \\
\hline & $1^{+}\left(1^{+-}\right)$ & $(1642,-139)$ & $b_{1}(1960)^{\dagger} ?$ & $1960 \pm 35$ & $230 \pm 50$ \\
\hline & $1^{-}\left(1^{++}\right)$ & $(1021,-251)$ & $a_{1}(1260)$ & $1230 \pm 40$ & $250 \sim 600$ \\
\hline & $1^{-}\left(1^{++}\right)$ & $(1568,-145)$ & $a_{1}(1640)^{\dagger}$ & $1647 \pm 22$ & $254 \pm 27$ \\
\hline \multirow[t]{2}{*}{$(0,1,2)$} & $1^{-}\left(2^{++}\right)$ & $(1228,0)$ & $a_{2}(1320)$ & $1318.3 \pm 0.6$ & $107 \pm 5$ \\
\hline & $1^{-}\left(2^{++}\right)$ & $(1775,-6)$ & $a_{2}(1700)^{\dagger}$ & $1732 \pm 16$ & $194 \pm 40$ \\
\hline \multirow[t]{3}{*}{$(1,1 / 2,0)$} & $1 / 2\left(0^{+}\right)$ & $(830,-170)$ & $K_{0}^{*}(800)^{\dagger}$ & $672 \pm 40$ & $550 \pm 34$ \\
\hline & $1 / 2\left(0^{+}\right)$ & $(1428,-24)$ & $K_{0}^{*}(1430)$ & $1425 \pm 50$ & $270 \pm 80$ \\
\hline & $1 / 2\left(0^{+}\right)$ & $(1787,-37)$ & $K_{0}^{*}(1950)^{\dagger}$ & $1945 \pm 22$ & $201 \pm 90$ \\
\hline \multirow[t]{4}{*}{$(1,1 / 2,1)$} & $1 / 2\left(1^{+}\right)$ & $(1188,-64)$ & $K_{1}(1270)$ & $1272 \pm 7$ & $90 \pm 20$ \\
\hline & $1 / 2\left(1^{+}\right)$ & $(1250,-31)$ & $K_{1}(\ldots)$ & & \\
\hline & $1 / 2\left(1^{+}\right)$ & $(1414,-66)$ & $K_{1}(1400) ?$ & $1403 \pm 7$ & $174 \pm 13$ \\
\hline & $1 / 2\left(1^{+}\right)$ & $(1665,-95)$ & $K_{1}(1650)^{\dagger} ?$ & $1650 \pm 50$ & $150 \pm 50$ \\
\hline$(1,1 / 2,2)$ & $1 / 2\left(2^{+}\right)$ & $(1708,-156)$ & $K_{2}^{*}(1430) ?$ & $1429 \pm 1.4$ & $104 \pm 4$ \\
\hline
\end{tabular}


TABLE XX: Poles with exotic quantum numbers found in this work and possible PDG counterparts. Units are given in MeV. Those resonances marked with $\dagger$ need to be confirmed.

\begin{tabular}{|c|c|c|c|c|c|}
\hline \multirow[t]{2}{*}{$(Y, I, J)$} & \multirow[t]{2}{*}{$I^{G}\left(J^{P C}\right)$} & \multirow{2}{*}{$\begin{array}{c}\text { This model } \\
\text { Pole position }\left(\sqrt{s_{R}}\right)\end{array}$} & \multicolumn{3}{|c|}{ PDG [84] } \\
\hline & & & Name & Mass & Width \\
\hline$(0,2,0)$ & $2^{+}\left(0^{++}\right)$ & $(1419,-54)$ & $X(1420)^{\dagger}$ & $1420 \pm 20$ & $160 \pm 10$ \\
\hline$(1,3 / 2,0)$ & $3 / 2\left(0^{+}\right)$ & $(1433,-70)$ & & & \\
\hline$(2,1,0)$ & $1\left(0^{+}\right)$ & $(1564,-66)$ & & & \\
\hline$(1,3 / 2,1)$ & $3 / 2\left(1^{+}\right)$ & $(1499,-127)$ & & & \\
\hline$(2,0,1)$ & $0\left(1^{+}\right)$ & $(1608,-114)$ & & & \\
\hline
\end{tabular}

of the resonances with these quantum numbers reported in the PDG and with masses below 2 GeV, it can be noted that there exists just one well established resonance that does not fit within the SU(6) classification pattern assumed in Table [ ${ }^{13}$ This is the $f_{0}(1500)$ resonance, for which a glueball picture has been suggested by several authors [103, 104]. Our result would then be in support of such picture.

(ii) $J^{P}=1^{+}$: Here the effects of the $\mathrm{SU}(6)$ breaking terms $\mathcal{D}_{m}$ and $\mathcal{D}_{m}$ terms turn out to be important. There are two types of channels, namely, $P V$ and $V V$ mesons coupled to total spin 1.

The $P V \rightarrow P V$ amplitudes are constrained by the LO WT theorem (see Eq. (54)) and give rise to the states of the multiplets $8_{3}$ and $1_{3}$ of the $\mathrm{SU}(6) \mathbf{3 5}_{s}$ irrep and to those of a further $\mathrm{SU}(3)$ octet $\left(8_{3}^{a}\right)$ of the $\mathrm{SU}(6) \mathbf{1 8 9}$. Note that the dynamics of the states of this latter multiplet is strongly influenced by the SU(6) breaking terms mentioned above. Our results for those multiplets are in good agreement with those previously obtained in Ref. [43], which among others include the prediction of the existence of a second $K_{1}(1270)$ resonance [109].

On the other hand the simultaneous consideration of $P V$ and $V V$ channels make the present approach different from that followed in Ref. [43], and has allowed us to dynamically generate also the $h_{1}(1595)$ resonance. The interference $P V \rightarrow V V$ amplitudes turn out to play a crucial role in producing this state, and that is presumably the reason why it is not generated either in the $V V \rightarrow V V$ study carried out in Ref. [70] using the formalism of the hidden gauge interaction for vector mesons. Possibly, the situation is similar for the $K_{1}(1650)$ state and thus we end up with a clearer $\mathrm{SU}(6)$ pattern, which is also followed to some extent in nature.

In this sector, we also predict two exotic states belonging to the 10 and $10^{*}$ irreps. On the other hand, we have verified that the missing $b_{1}$ pole in the symmetric octet of the SU(6) $\mathbf{1 8 9}$ would appear if the SU(6) symmetry were restored.

To finish the discussion of this sector, we would like to point out that below $2 \mathrm{GeV}$ there is only one firmly established axial vector resonance that does not fit in the symmetry pattern sketched in Table I It is the $f_{1}(1420)$, and similarly to the previous discussion for the $f_{0}(1500)$ resonance, this might hint at the possible existence of gluon components in its wave-function. Indeed arguments favoring the $f_{1}(1420)$ being a hybrid $q \bar{q} g$ meson have been put forward by Ishida and collaborators [112].

(iii) $J^{P}=2^{+}$: In this sector is where the $\mathrm{SU}(6)$ pattern works worst. This is because the $\mathrm{SU}(6)$ symmetry kinetic term becomes less dominant when compared to $V V$ interaction contact term generated as a result of giving mass to the vector mesons. Moreover, we must stress here, once more, the little control that we have over this term. Yet, the interaction in the $\mathrm{SU}(6) \mathbf{1 8 9}$ irrep associated to $\mathcal{D}_{\text {kin }}$ is relatively weak. Thus all results displayed in Table \ for this sector must be understood by actively considering the interplay between $\mathcal{D}_{\text {kin }}$ and $\mathcal{D}_{m}$. The first remark is that if the contact term is switched off, the pole associated to the $f_{2}(1270)$ moves up in mass by more than $200 \mathrm{MeV}$ and the $a_{2}(1320)$ resonance disappears. Actually, in each $Y I$ subsector, $\mathcal{D}_{m}$ has two large and negative (attractive) eigenvalues, which correspond to a full nonet (singlet plus octet). The $a_{2}(1320)$

\footnotetext{
${ }^{13}$ We will omit here any reference to the $K(1630)$ resonance, since its $J^{P}$ is undetermined yet [84].
} 
would be part of this nonet, and it might well be that the actual $K_{2}^{*}(1430)$ could be also a member of it. In that scenario, the pole at $(1708,-156)$ obtained here, and that we cannot move down closer to the mass of the $K_{2}^{*}(1430)$ resonance, might correspond to a further state, for which we do not find an easy correspondence with any of those reported in the PDG. On the other hand, by changing the subtraction constants it is possible to generate some more $0^{+}\left(2^{++}\right)$poles within our scheme, which might account for those states needed to fill in completely the nonet mentioned above.

In this sector, and in contrast to the $0^{+}$and $1^{+}$cases, there appear in the PDG several even parity resonances that cannot be accommodated within our scheme. Some of them, might be glueballs, but we cannot be here as precise as we were in the previous sectors. The hidden gauge formalism for vector mesons used in Ref. [70] does not improve on that, though its choice for the contact $V V$ term might provide a more robust description of the $f_{2}(1270)$ and $K_{2}^{*}(1430)$ resonances than that obtained here.

In summary, it has been shown that most of the low-lying even parity meson resonances, specially in the $J^{P}=0^{+}$ and $1^{+}$sectors, can be classified according to multiplets of the spin-flavor symmetry group SU(6). The $f_{0}(1500)$, $f_{1}(1420)$ and some $0^{+}\left(2^{++}\right)$resonances cannot be accommodated within SU(6) multiplets and thus they are clear candidates to be glueballs or hybrids. On the other hand, we predict the existence of five exotic resonances $(I \geq 3 / 2$ and/or $|Y|=2$ ) with masses in region $1.4-1.6 \mathrm{GeV}$, which would complete the $27_{1}$ and $10_{3}$ and $10_{3}^{*}$ spin-flavor multiplets.

\section{Acknowledgments}

We warmly thank E. Oset and E. Ruiz-Arriola for useful discussions. This research was supported by DGI and FEDER funds, under contracts FIS2008-01143, FIS2006-03438, by the EU contract FLAVIAnet MRTN-CT-2006035482, the Spanish Consolider-Ingenio 2010 Programme CPAN (CSD2007-00042), and the Junta de Andalucía grants FQM225 and it is part of the European Community-Research Infrastructure Integrating Activity "Study of Strongly Interacting Matter" (acronym HadronPhysics2, Grant Agreement n. 227431) under the Seventh Framework Programme of EU. LSG acknowledges support from the MICINN in the Program "Juan de la Cierva" and from the Alexander von Humboldt foundation through a research fellowship. 


\section{Appendix A: Coefficients of the $s$-wave tree level amplitudes}

This Appendix gives the $\mathcal{D}_{\text {kin }}, \mathcal{D}_{m}$ and $\mathcal{D}_{a}$ matrices of the $s$-wave tree level meson-meson amplitudes in Eq. (40), for the various $Y I J$ sectors (Tables XXI LXI).

\section{Kinetic term: $\mathcal{D}_{\text {kin }}$}

TABLE XXI: $(Y, I, J)=(0,0,0)$.

\begin{tabular}{|c|c|c|c|c|c|c|c|}
\hline$\pi \pi$ & $\bar{K} K$ & $\eta \eta$ & $\rho \rho$ & $\omega \omega$ & $\omega \phi$ & $\bar{K}^{*} K^{*}$ & $\phi \phi$ \\
\hline-2 & $\frac{\sqrt{3}}{2}$ & 0 & $2 \sqrt{3}$ & 0 & 0 & $-\frac{3}{2}$ & 0 \\
\hline$\frac{\sqrt{3}}{2}$ & $-\frac{3}{2}$ & $-\frac{3}{2}$ & $-\frac{3}{2}$ & $\frac{\sqrt{3}}{2}$ & $\sqrt{3}$ & $\frac{3 \sqrt{3}}{2}$ & $\sqrt{3}$ \\
\hline 0 & $-\frac{3}{2}$ & 0 & 0 & 0 & 0 & $\frac{3 \sqrt{3}}{2}$ & 0 \\
\hline $2 \sqrt{3}$ & $-\frac{3}{2}$ & 0 & -4 & $2 \sqrt{3}$ & 0 & $\frac{3 \sqrt{3}}{2}$ & 0 \\
\hline 0 & $\frac{\sqrt{3}}{2}$ & 0 & $2 \sqrt{3}$ & -2 & 0 & $-\frac{3}{2}$ & 0 \\
\hline 0 & $\sqrt{3}$ & 0 & 0 & 0 & 0 & 1 & 0 \\
\hline$-\frac{3}{2}$ & $\frac{3 \sqrt{3}}{2}$ & $\frac{3 \sqrt{3}}{2}$ & $\frac{3 \sqrt{3}}{2}$ & $-\frac{3}{2}$ & 1 & $-\frac{9}{2}$ & -3 \\
\hline 0 & $\sqrt{3}$ & 0 & 0 & 0 & 0 & -3 & -4 \\
\hline
\end{tabular}

TABLE XXII: $(Y, I, J)=(0,0,1)$.

\begin{tabular}{|c|c|c|c|c|c|c|c|}
\hline$G$ & $\eta \phi$ & $\eta \omega$ & $\pi \rho$ & $\left(\bar{K} K^{*}\right)_{A}$ & $\bar{K}^{*} K^{*}$ & $\omega \phi$ & $\left(\bar{K} K^{*}\right)_{S}$ \\
\hline- & 0 & 0 & 0 & $-\sqrt{6}$ & $-\sqrt{6}$ & & \\
\hline- & 0 & 0 & 0 & $-\sqrt{3}$ & $\sqrt{3}$ & & \\
\hline- & 0 & 0 & -4 & $\sqrt{3}$ & $-\sqrt{3}$ & & \\
\hline- & $-\sqrt{6}$ & $-\sqrt{3}$ & $\sqrt{3}$ & -3 & -1 & & \\
\hline- & $-\sqrt{6}$ & $\sqrt{3}$ & $-\sqrt{3}$ & -1 & -3 & & \\
\hline+ & & & & & & 0 & -2 \\
\hline+ & & & & & & -2 & 0 \\
\hline
\end{tabular}


TABLE XXIII: $(Y, I, J)=(0,0,2)$.

\begin{tabular}{c|ccccc}
\hline \hline & $\rho \rho$ & $\omega \omega$ & $\omega \phi$ & $\bar{K}^{*} K^{*}$ & $\phi \phi$ \\
\hline & -1 & $-\sqrt{3}$ & 0 & 0 & 0 \\
$-\sqrt{3}$ & 1 & 0 & 0 & 0 \\
0 & 0 & 0 & -2 & 0 \\
& 0 & -2 & 0 & 0 \\
& 0 & 0 & 0 & 0 & 2 \\
\hline \hline
\end{tabular}

TABLE XXIV: $(Y, I, J)=(0,1,0)$.

\begin{tabular}{c|ccccc}
\hline \hline & $\eta \pi$ & $\bar{K} K$ & $\omega \rho$ & $\phi \rho$ & $\bar{K}^{*} K^{*}$ \\
\hline \multirow{7}{*}{$\sqrt{\frac{3}{2}}$} & $-\frac{1}{2}$ & 0 & 0 & $-\frac{3}{\sqrt{2}}$ \\
& 0 & $-\sqrt{\frac{3}{2}}$ & -4 & 0 & $\frac{3}{\sqrt{2}}$ \\
& 0 & $-\sqrt{3}$ & 0 & 0 & -1 \\
& $-\frac{3}{\sqrt{2}}$ & $\frac{\sqrt{3}}{2}$ & $\frac{3}{\sqrt{2}}$ & -1 & $-\frac{3}{2}$ \\
\hline \hline
\end{tabular}

TABLE XXV: $(Y, I, J)=(0,1,1)$.

\begin{tabular}{|c|c|c|c|c|c|c|c|c|c|c|}
\hline$G$ & $\pi \phi$ & $\pi \omega$ & $\eta \rho$ & $\left(\bar{K} K^{*}\right)_{S}$ & $\rho \rho$ & $\bar{K}^{*} K^{*}$ & $\pi \rho$ & $\left(\bar{K} K^{*}\right)_{A}$ & $\omega \rho$ & $\phi \rho$ \\
\hline+ & 0 & 0 & 0 & $\sqrt{2}$ & 0 & $\sqrt{2}$ & & & & \\
\hline+ & 0 & 0 & 0 & 1 & $2 \sqrt{2}$ & -1 & & & & \\
\hline+ & 0 & 0 & 0 & $\sqrt{3}$ & 0 & $-\sqrt{3}$ & & & & \\
\hline+ & $\sqrt{2}$ & 1 & $\sqrt{3}$ & -1 & $-\sqrt{2}$ & 1 & & & & \\
\hline+ & 0 & $2 \sqrt{2}$ & 0 & $-\sqrt{2}$ & -2 & $\sqrt{2}$ & & & & \\
\hline+ & $\sqrt{2}$ & -1 & $-\sqrt{3}$ & 1 & $\sqrt{2}$ & -1 & & & & \\
\hline- & & & & & & & 0 & 0 & -2 & 0 \\
\hline- & & & & & & & 0 & 0 & 0 & -2 \\
\hline- & & & & & & & -2 & 0 & 0 & 0 \\
\hline- & & & & & & & 0 & -2 & 0 & 0 \\
\hline
\end{tabular}


TABLE XXVI: $(Y, I, J)=(0,1,2)$.

\begin{tabular}{c|ccc}
\hline \hline & $\omega \rho$ & $\phi \rho$ & $\bar{K}^{*} K^{*}$ \\
\hline & 2 & 0 & 0 \\
& 0 & 0 & 2 \\
& 0 & 2 & 0 \\
\hline \hline
\end{tabular}

TABLE XXVII: $(Y, I, J)=(0,2,0)$.

\begin{tabular}{c|cc}
\hline \hline & $\pi \pi$ & $\rho \rho$ \\
\hline & 1 & $-\sqrt{3}$ \\
& $-\sqrt{3}$ & -1 \\
\hline \hline
\end{tabular}

TABLE XXVIII: $(Y, I, J)=(0,2,1)$.

\begin{tabular}{l|c}
\hline \hline & $\pi \rho$ \\
\hline & 2 \\
\hline \hline
\end{tabular}

TABLE XXIX: $(Y, I, J)=(0,2,2)$.

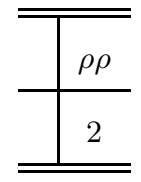

TABLE XXX: $(Y, I, J)=(1,1 / 2,0)$.

\begin{tabular}{c|ccccc}
\hline \hline & $K \pi$ & $\eta K$ & $K^{*} \rho$ & $K^{*} \omega$ & $K^{*} \phi$ \\
\hline$-\frac{5}{4}$ & $\frac{3}{4}$ & $\frac{5 \sqrt{3}}{4}$ & $-\frac{3}{4}$ & $-\frac{3}{2 \sqrt{2}}$ \\
$\frac{3}{4}$ & $\frac{3}{4}$ & $-\frac{3 \sqrt{3}}{4}$ & $-\frac{3}{4}$ & $-\frac{3}{2 \sqrt{2}}$ \\
& $\frac{5 \sqrt{3}}{4}$ & $-\frac{3 \sqrt{3}}{4}$ & $-\frac{7}{4}$ & $-\frac{5 \sqrt{3}}{4}$ & $\frac{3 \sqrt{\frac{3}{2}}}{2}$ \\
$-\frac{3}{4}$ & $-\frac{3}{4}$ & $-\frac{5 \sqrt{3}}{4}$ & $-\frac{5}{4}$ & $\frac{3}{2 \sqrt{2}}$ \\
$-\frac{3}{2 \sqrt{2}}$ & $-\frac{3}{2 \sqrt{2}}$ & $\frac{3 \sqrt{\frac{3}{2}}}{2}$ & $\frac{3}{2 \sqrt{2}}$ & $-\frac{5}{2}$ \\
\hline \hline
\end{tabular}


TABLE XXXI: $(Y, I, J)=(1,1 / 2,1)$.

\begin{tabular}{|c|c|c|c|c|c|c|c|}
\hline$\pi K^{*}$ & $K \rho$ & $K \omega$ & $\eta K^{*}$ & $K \phi$ & $K^{*} \rho$ & $K^{*} \omega$ & $K^{*} \phi$ \\
\hline$-\frac{5}{4}$ & $\frac{5}{4}$ & $-\frac{\sqrt{3}}{4}$ & $-\frac{3}{4}$ & $-\frac{\sqrt{\frac{3}{2}}}{2}$ & $-\frac{5}{2 \sqrt{2}}$ & $\frac{\sqrt{\frac{3}{2}}}{2}$ & $-\frac{\sqrt{3}}{2}$ \\
\hline$\frac{5}{4}$ & $-\frac{5}{4}$ & $\frac{\sqrt{3}}{4}$ & $\frac{3}{4}$ & $\frac{\sqrt{\frac{3}{2}}}{2}$ & $\frac{1}{2 \sqrt{2}}$ & $\frac{3 \sqrt{\frac{3}{2}}}{2}$ & $\frac{\sqrt{3}}{2}$ \\
\hline$-\frac{\sqrt{3}}{4}$ & $\frac{\sqrt{3}}{4}$ & $\frac{1}{4}$ & $\frac{\sqrt{3}}{4}$ & $\frac{1}{2 \sqrt{2}}$ & $\frac{3 \sqrt{\frac{3}{2}}}{2}$ & $\frac{3}{2 \sqrt{2}}$ & $\frac{1}{2}$ \\
\hline$-\frac{3}{4}$ & $\frac{3}{4}$ & $\frac{\sqrt{3}}{4}$ & $\frac{3}{4}$ & $\frac{\sqrt{\frac{3}{2}}}{2}$ & $-\frac{3}{2 \sqrt{2}}$ & $-\frac{\sqrt{\frac{3}{2}}}{2}$ & $\frac{\sqrt{3}}{2}$ \\
\hline$-\frac{\sqrt{\frac{3}{2}}}{2}$ & $\frac{\sqrt{\frac{3}{2}}}{2}$ & $\frac{1}{2 \sqrt{2}}$ & $\frac{\sqrt{\frac{3}{2}}}{2}$ & $\frac{1}{2}$ & $-\frac{\sqrt{3}}{2}$ & $-\frac{1}{2}$ & $-\frac{3}{\sqrt{2}}$ \\
\hline$-\frac{5}{2 \sqrt{2}}$ & $\frac{1}{2 \sqrt{2}}$ & $\frac{3 \sqrt{\frac{3}{2}}}{2}$ & $-\frac{3}{2 \sqrt{2}}$ & $-\frac{\sqrt{3}}{2}$ & $-\frac{3}{2}$ & $-\frac{\sqrt{3}}{2}$ & $-\sqrt{\frac{3}{2}}$ \\
\hline$\frac{\sqrt{\frac{3}{2}}}{2}$ & $\frac{3 \sqrt{\frac{3}{2}}}{2}$ & $\frac{3}{2 \sqrt{2}}$ & $-\frac{\sqrt{\frac{3}{2}}}{2}$ & $-\frac{1}{2}$ & $-\frac{\sqrt{3}}{2}$ & $-\frac{1}{2}$ & $-\frac{1}{\sqrt{2}}$ \\
\hline$-\frac{\sqrt{3}}{2}$ & $\frac{\sqrt{3}}{2}$ & $\frac{1}{2}$ & $\frac{\sqrt{3}}{2}$ & $-\frac{3}{\sqrt{2}}$ & $-\sqrt{\frac{3}{2}}$ & $-\frac{1}{\sqrt{2}}$ & -1 \\
\hline
\end{tabular}

TABLE XXXII: $(Y, I, J)=(1,1 / 2,2)$.

\begin{tabular}{c|ccc}
\hline \hline & $K^{*} \rho$ & $K^{*} \omega$ & $K^{*} \phi$ \\
\hline & -1 & $\sqrt{3}$ & 0 \\
& $\sqrt{3}$ & 1 & 0 \\
& 0 & 0 & 2 \\
\hline \hline
\end{tabular}

TABLE XXXIII: $(Y, I, J)=(1,3 / 2,0)$.

\begin{tabular}{c|cc}
\hline \hline & $K \pi$ & $K^{*} \rho$ \\
\hline & 1 & $-\sqrt{3}$ \\
& $-\sqrt{3}$ & -1 \\
\hline \hline
\end{tabular}

TABLE XXXIV: $(Y, I, J)=(1,3 / 2,1)$.

\begin{tabular}{c|ccc}
\hline \hline & $\pi K^{*}$ & $K \rho$ & $K^{*} \rho$ \\
\hline 1 & 1 & $-\sqrt{2}$ \\
1 & 1 & $\sqrt{2}$ \\
& $-\sqrt{2}$ & $\sqrt{2}$ & 0 \\
\hline \hline
\end{tabular}

TABLE XXXV: $(Y, I, J)=(1,3 / 2,2)$.

\begin{tabular}{c|c}
\hline \hline & $K^{*} \rho$ \\
\hline & 2 \\
\hline \hline
\end{tabular}


TABLE XXXVI: $(Y, I, J)=(2,0,1)$.

\begin{tabular}{c|cc}
\hline \hline & $K K^{*}$ & $K^{*} K^{*}$ \\
\hline & 0 & -2 \\
& -2 & 0 \\
\hline \hline
\end{tabular}

TABLE XXXVII: $(Y, I, J)=(2,1,0)$.

\begin{tabular}{c|cc}
\hline \hline & $K K$ & $K^{*} K^{*}$ \\
\hline & 1 & $-\sqrt{3}$ \\
& $-\sqrt{3}$ & -1 \\
\hline \hline
\end{tabular}

TABLE XXXVIII: $(Y, I, J)=(2,1,1)$.

\begin{tabular}{c|c}
\hline \hline & $K K^{*}$ \\
\hline & 2 \\
\hline \hline
\end{tabular}

TABLE XXXIX: $(Y, I, J)=(2,1,2)$.

\begin{tabular}{c|c}
\hline \hline & $K^{*} K^{*}$ \\
\hline & 2 \\
\hline \hline
\end{tabular}


2. Contact term: $\mathcal{D}_{m}$

TABLE XL: $(Y, I, J)=(0,0,0)$.

\begin{tabular}{|c|c|c|c|c|c|c|c|}
\hline$\pi \pi$ & $\bar{K} K$ & $\eta \eta$ & $\rho \rho$ & $\omega \omega$ & $\omega \phi$ & $\bar{K}^{*} K^{*}$ & $\phi \phi$ \\
\hline 0 & 0 & 0 & $\frac{16}{\sqrt{3}}$ & 0 & 0 & -4 & 0 \\
\hline 0 & 0 & 0 & -4 & $\frac{4}{\sqrt{3}}$ & $\frac{8}{\sqrt{3}}$ & $4 \sqrt{3}$ & $\frac{8}{\sqrt{3}}$ \\
\hline 0 & 0 & 0 & 0 & 0 & 0 & $4 \sqrt{3}$ & 0 \\
\hline$\frac{16}{\sqrt{3}}$ & -4 & 0 & $-\frac{208}{3}$ & $\frac{80}{\sqrt{3}}$ & 0 & $24 \sqrt{3}$ & 0 \\
\hline 0 & $\frac{4}{\sqrt{3}}$ & 0 & $\frac{80}{\sqrt{3}}$ & $-\frac{80}{3}$ & 0 & -24 & 0 \\
\hline 0 & $\frac{8}{\sqrt{3}}$ & 0 & 0 & 0 & 0 & $\frac{16}{3}$ & 0 \\
\hline-4 & $4 \sqrt{3}$ & $4 \sqrt{3}$ & $24 \sqrt{3}$ & -24 & $\frac{16}{3}$ & -72 & -48 \\
\hline 0 & $\frac{8}{\sqrt{3}}$ & 0 & 0 & 0 & 0 & -48 & $-\frac{160}{3}$ \\
\hline
\end{tabular}

TABLE XLI: $(Y, I, J)=(0,0,1)$.

\begin{tabular}{|c|c|c|c|c|c|c|c|}
\hline$G$ & $\eta \phi$ & $\eta \omega$ & $\pi \rho$ & $\left(\bar{K} K^{*}\right)_{A}$ & $\bar{K}^{*} K^{*}$ & $\omega \phi$ & $\left(\bar{K} K^{*}\right)_{S}$ \\
\hline- & 0 & 0 & 0 & $4 \sqrt{\frac{2}{3}}$ & 0 & & \\
\hline- & 0 & 0 & 0 & $\frac{4}{\sqrt{3}}$ & 0 & & \\
\hline- & 0 & 0 & $\frac{16}{3}$ & $-\frac{4}{\sqrt{3}}$ & 0 & & \\
\hline- & $4 \sqrt{\frac{2}{3}}$ & $\frac{4}{\sqrt{3}}$ & $-\frac{4}{\sqrt{3}}$ & 4 & 0 & & \\
\hline- & 0 & 0 & 0 & 0 & -28 & & \\
\hline+ & & & & & & 0 & 0 \\
\hline+ & & & & & & 0 & -12 \\
\hline
\end{tabular}


TABLE XLII: $(Y, I, J)=(0,0,2)$.

\begin{tabular}{c|ccccc}
\hline \hline & $\rho \rho$ & $\omega \omega$ & $\omega \phi$ & $\bar{K}^{*} K^{*}$ & $\phi \phi$ \\
\hline & $-\frac{16}{3}$ & $\frac{32}{\sqrt{3}}$ & 0 & $4 \sqrt{3}$ & 0 \\
$\frac{32}{\sqrt{3}}$ & $-\frac{32}{3}$ & 0 & -4 & 0 \\
0 & 0 & 0 & $\frac{40}{3}$ & 0 \\
& $4 \sqrt{3}$ & -4 & $\frac{40}{3}$ & -12 & -8 \\
& 0 & 0 & 0 & -8 & $-\frac{64}{3}$ \\
\hline \hline
\end{tabular}

TABLE XLIII: $(Y, I, J)=(0,1,0)$.

\begin{tabular}{c|ccccc}
\hline \hline & $\eta \pi$ & $\bar{K} K$ & $\omega \rho$ & $\phi \rho$ & $\bar{K}^{*} K^{*}$ \\
\hline & 0 & 0 & 0 & 0 & $-4 \sqrt{2}$ \\
0 & 0 & $-4 \sqrt{\frac{2}{3}}$ & $-\frac{8}{\sqrt{3}}$ & $\frac{4}{\sqrt{3}}$ \\
0 & $-4 \sqrt{\frac{2}{3}}$ & $-\frac{160}{3}$ & 0 & $24 \sqrt{2}$ \\
& $-\frac{8}{\sqrt{3}}$ & 0 & 0 & $-\frac{16}{3}$ \\
& $-4 \sqrt{2}$ & $\frac{4}{\sqrt{3}}$ & $24 \sqrt{2}$ & $-\frac{16}{3}$ & -24 \\
\hline \hline
\end{tabular}

TABLE XLIV: $(Y, I, J)=(0,1,1)$.

\begin{tabular}{|c|c|c|c|c|c|c|c|c|c|c|}
\hline$G$ & $\pi \phi$ & $\pi \omega$ & $\eta \rho$ & $\left(\bar{K} K^{*}\right)_{S}$ & $\rho \rho$ & $\bar{K}^{*} K^{*}$ & $\pi \rho$ & $\left(\bar{K} K^{*}\right)_{A}$ & $\omega \rho$ & $\phi \rho$ \\
\hline+ & 0 & 0 & 0 & $-\frac{4 \sqrt{2}}{3}$ & 0 & 0 & & & & \\
\hline+ & 0 & 0 & 0 & $-\frac{4}{3}$ & 0 & 0 & & & & \\
\hline+ & 0 & 0 & 0 & $-\frac{4}{\sqrt{3}}$ & 0 & 0 & & & & \\
\hline+ & $-\frac{4 \sqrt{2}}{3}$ & $-\frac{4}{3}$ & $-\frac{4}{\sqrt{3}}$ & $\frac{4}{3}$ & 0 & 0 & & & & \\
\hline+ & 0 & 0 & 0 & 0 & $-\frac{56}{3}$ & $\frac{28 \sqrt{2}}{3}$ & & & & \\
\hline+ & 0 & 0 & 0 & 0 & $\frac{28 \sqrt{2}}{3}$ & $-\frac{28}{3}$ & & & & \\
\hline- & & & & & & & -8 & $4 \sqrt{2}$ & 0 & 0 \\
\hline- & & & & & & & $4 \sqrt{2}$ & -4 & 0 & 0 \\
\hline- & & & & & & & 0 & 0 & 0 & 0 \\
\hline- & & & & & & & 0 & 0 & 0 & 0 \\
\hline
\end{tabular}


TABLE XLV: $(Y, I, J)=(0,1,2)$.

\begin{tabular}{c|ccc}
\hline \hline & $\omega \rho$ & $\phi \rho$ & $\bar{K}^{*} K^{*}$ \\
\hline & $-\frac{64}{3}$ & 0 & $4 \sqrt{2}$ \\
0 & 0 & $-\frac{40}{3}$ \\
& $4 \sqrt{2}$ & $-\frac{40}{3}$ & -4 \\
\hline \hline
\end{tabular}

TABLE XLVI: $(Y, I, J)=(0,2,0)$.

\begin{tabular}{c|cc}
\hline \hline & $\pi \pi$ & $\rho \rho$ \\
\hline & 0 & $-\frac{8}{\sqrt{3}}$ \\
& $-\frac{8}{\sqrt{3}}$ & $-\frac{16}{3}$ \\
\hline \hline
\end{tabular}

TABLE XLVII: $(Y, I, J)=(0,2,1)$.

\begin{tabular}{c|c}
\hline \hline & $\pi \rho$ \\
\hline & $-\frac{8}{3}$ \\
\hline \hline
\end{tabular}

TABLE XLVIII: $(Y, I, J)=(0,2,2)$.

\begin{tabular}{c|c}
\hline \hline & $\rho \rho$ \\
\hline & $-\frac{40}{3}$ \\
\hline \hline
\end{tabular}

TABLE XLIX: $(Y, I, J)=(1,1 / 2,0)$.

\begin{tabular}{c|ccccc}
\hline \hline & $K \pi$ & $\eta K$ & $K^{*} \rho$ & $K^{*} \omega$ & $K^{*} \phi$ \\
\hline 0 & 0 & $\frac{10}{\sqrt{3}}$ & -2 & $-2 \sqrt{2}$ \\
0 & 0 & $-2 \sqrt{3}$ & -2 & $-2 \sqrt{2}$ \\
& $\frac{10}{\sqrt{3}}$ & $-2 \sqrt{3}$ & $-\frac{100}{3}$ & $-\frac{44}{\sqrt{3}}$ & $12 \sqrt{6}$ \\
-2 & -2 & $-\frac{44}{\sqrt{3}}$ & $-\frac{44}{3}$ & $12 \sqrt{2}$ \\
& $-2 \sqrt{2}$ & $-2 \sqrt{2}$ & $12 \sqrt{6}$ & $12 \sqrt{2}$ & $-\frac{88}{3}$ \\
\hline \hline
\end{tabular}


TABLE L: $(Y, I, J)=(1,1 / 2,1)$.

\begin{tabular}{|c|c|c|c|c|c|c|c|}
\hline$\pi K^{*}$ & $K \rho$ & $K \omega$ & $\eta K^{*}$ & $K \phi$ & $K^{*} \rho$ & $K^{*} \omega$ & $K^{*} \phi$ \\
\hline$-\frac{4}{3}$ & $-\frac{14}{3}$ & $-\frac{2}{\sqrt{3}}$ & 4 & $-2 \sqrt{\frac{2}{3}}$ & 0 & 0 & 0 \\
\hline$-\frac{14}{3}$ & $-\frac{4}{3}$ & $-\frac{4}{\sqrt{3}}$ & 2 & $-4 \sqrt{\frac{2}{3}}$ & 0 & 0 & 0 \\
\hline$-\frac{2}{\sqrt{3}}$ & $-\frac{4}{\sqrt{3}}$ & $-\frac{4}{3}$ & $\frac{2}{\sqrt{3}}$ & $-\frac{4 \sqrt{2}}{3}$ & 0 & 0 & 0 \\
\hline 4 & 2 & $\frac{2}{\sqrt{3}}$ & -4 & $2 \sqrt{\frac{2}{3}}$ & 0 & 0 & 0 \\
\hline$-2 \sqrt{\frac{2}{3}}$ & $-4 \sqrt{\frac{2}{3}}$ & $-\frac{4 \sqrt{2}}{3}$ & $2 \sqrt{\frac{2}{3}}$ & $-\frac{8}{3}$ & 0 & 0 & 0 \\
\hline 0 & 0 & 0 & 0 & 0 & -14 & $-\frac{14}{\sqrt{3}}$ & $-14 \sqrt{\frac{2}{3}}$ \\
\hline 0 & 0 & 0 & 0 & 0 & $-\frac{14}{\sqrt{3}}$ & $-\frac{14}{3}$ & $-\frac{14 \sqrt{2}}{3}$ \\
\hline 0 & 0 & 0 & 0 & 0 & $-14 \sqrt{\frac{2}{3}}$ & $-\frac{14 \sqrt{2}}{3}$ & $-\frac{28}{3}$ \\
\hline
\end{tabular}

TABLE LI: $(Y, I, J)=(1,1 / 2,2)$.

\begin{tabular}{c|ccc}
\hline \hline & $K^{*} \rho$ & $K^{*} \omega$ & $K^{*} \phi$ \\
\hline & $\frac{2}{3}$ & $-\frac{26}{\sqrt{3}}$ & $2 \sqrt{6}$ \\
& $-\frac{26}{\sqrt{3}}$ & $-\frac{26}{3}$ & $2 \sqrt{2}$ \\
& $2 \sqrt{6}$ & $2 \sqrt{2}$ & $-\frac{52}{3}$ \\
\hline \hline
\end{tabular}

TABLE LII: $(Y, I, J)=(1,3 / 2,0)$.

\begin{tabular}{c|cc}
\hline \hline & $K \pi$ & $K^{*} \rho$ \\
\hline & 0 & $-\frac{8}{\sqrt{3}}$ \\
& $-\frac{8}{\sqrt{3}}$ & $-\frac{16}{3}$ \\
\hline \hline
\end{tabular}

TABLE LIII: $(Y, I, J)=(1,3 / 2,1)$.

\begin{tabular}{c|ccc}
\hline \hline & $\pi K^{*}$ & $K \rho$ & $K^{*} \rho$ \\
\hline & $-\frac{4}{3}$ & $-\frac{4}{3}$ & 0 \\
& $-\frac{4}{3}$ & $-\frac{4}{3}$ & 0 \\
& 0 & 0 & 0 \\
\hline
\end{tabular}

TABLE LIV: $(Y, I, J)=(1,3 / 2,2)$.

\begin{tabular}{l|l}
\hline \hline & $K^{*} \rho$ \\
\hline & $-\frac{40}{3}$ \\
\hline \hline
\end{tabular}


TABLE LV: $(Y, I, J)=(2,0,1)$.

\begin{tabular}{c|cc}
\hline \hline & $K K^{*}$ & $K^{*} K^{*}$ \\
\hline & 0 & 0 \\
& 0 & 0 \\
\hline \hline
\end{tabular}

TABLE LVI: $(Y, I, J)=(2,1,0)$.

\begin{tabular}{c|cc}
\hline \hline & $K K$ & $K^{*} K^{*}$ \\
\hline & 0 & $-\frac{8}{\sqrt{3}}$ \\
& $-\frac{8}{\sqrt{3}}$ & $-\frac{16}{3}$ \\
\hline \hline
\end{tabular}

TABLE LVII: $(Y, I, J)=(2,1,1)$.

\begin{tabular}{l|l}
\hline \hline & $K K^{*}$ \\
\hline & $-\frac{8}{3}$ \\
\hline \hline
\end{tabular}

TABLE LVIII: $(Y, I, J)=(2,1,2)$.

\begin{tabular}{l|l}
\hline \hline & $K^{*} K^{*}$ \\
\hline & $-\frac{40}{3}$ \\
\hline \hline
\end{tabular}


3. $(u-t)$ term: $\mathcal{D}_{a}$

The matrix elements corresponding to $\mathcal{D}_{a}$ are displayed. The $(Y, I, J)$ or $(Y, I, J, G)$ sectors with identically zero matrices are omitted. $P P$ and $V V$ channels (for which the matrix vanishes) are also omitted.

TABLE LIX: $(Y, I, J, G)=(0,0,1,+)$.

\begin{tabular}{c|c}
\hline \hline & $\left(\bar{K} K^{*}\right)_{S}$ \\
\hline & 3 \\
\hline \hline
\end{tabular}

TABLE LX: $(Y, I, J, G)=(0,1,1,-)$.

\begin{tabular}{c|cc}
\hline \hline & $\pi \rho$ & $\left(\bar{K} K^{*}\right)_{A}$ \\
\hline & 2 & $-\sqrt{2}$ \\
& $-\sqrt{2}$ & 1 \\
\hline \hline
\end{tabular}

TABLE LXI: $(Y, I, J)=(1,1 / 2,1)$.

\begin{tabular}{c|ccccc}
\hline \hline & $\pi K^{*}$ & $K \rho$ & $K \omega$ & $\eta K^{*}$ & $K \phi$ \\
\hline & $\frac{3}{4}$ & $\frac{3}{4}$ & $\frac{\sqrt{3}}{4}$ & $-\frac{3}{4}$ & $\frac{\sqrt{\frac{3}{2}}}{2}$ \\
& $\frac{3}{4}$ & $\frac{3}{4}$ & $\frac{\sqrt{3}}{4}$ & $-\frac{3}{4}$ & $\frac{\sqrt{\frac{3}{2}}}{2}$ \\
& $\frac{\sqrt{3}}{4}$ & $\frac{\sqrt{3}}{4}$ & $\frac{1}{4}$ & $-\frac{\sqrt{3}}{4}$ & $\frac{1}{2 \sqrt{2}}$ \\
& $-\frac{3}{4}$ & $-\frac{3}{4}$ & $-\frac{\sqrt{3}}{4}$ & $\frac{3}{4}$ & $-\frac{\sqrt{\frac{3}{2}}}{2}$ \\
& $\frac{\sqrt{\frac{3}{2}}}{2}$ & $\frac{\sqrt{\frac{3}{2}}}{2}$ & $\frac{1}{2 \sqrt{2}}$ & $-\frac{\sqrt{\frac{3}{2}}}{2}$ & $\frac{1}{2}$ \\
\hline \hline
\end{tabular}




\section{Appendix B: Computation of the eigenvalues}

To get the eigenvalues associated to each of the $\mathrm{SU}(6)$ projectors in Eqs. (34) and (35), we just let act $\mathcal{H}_{ \pm}^{\mathrm{SU}(6)}$ on one of the states of the vector space associated to each representation. For instance, the vector space associated to the singlet representation is generated by the state

$$
|1\rangle=M_{b}^{\dagger a} M_{a}^{\dagger b}|0\rangle, \quad a, b=1, \cdots 2 N_{F} .
$$

This gives

$$
\begin{aligned}
\hat{\mathcal{G}}_{+}|1\rangle & =: \operatorname{Tr}\left(\left(M M^{\dagger}\right)^{2}-M^{2} M^{\dagger 2}\right): M_{b}^{\dagger a} M_{a}^{\dagger b}|0\rangle \\
& =2(M_{j}^{\dagger i} M_{k}^{j} M_{l}^{\dagger k} \underbrace{l}_{i} M_{b}^{\dagger a} M_{a}^{\dagger b}-M_{j}^{\dagger i} M_{k}^{\dagger j} \underbrace{k}_{l} \underbrace{l}_{i} M_{b}^{\dagger a} M_{a}^{\dagger b})|0\rangle=-4 N_{F}|1\rangle
\end{aligned}
$$

and similarly for $\hat{\mathcal{G}}_{\mathcal{M}}$. For the remaining symmetric representations, a convenient choice of states is

$$
|2\rangle=M_{b}^{\dagger 1} M_{2}^{\dagger b}|0\rangle, \quad|3\rangle=\left(M_{2}^{\dagger 1} M_{4}^{\dagger 3}-M_{2}^{\dagger 3} M_{4}^{\dagger 1}\right)|0\rangle, \quad|4\rangle=M_{2}^{\dagger 1} M_{2}^{\dagger 1}|0\rangle
$$

for the $\mathbf{3 5}, \mathbf{1 8 9}$ and $\mathbf{4 0 5}$ representations, respectively.

The $p$-wave part $\mathcal{G}_{d}-\mathcal{G}_{c}$ can be represented as the matrix element of an operator in a fermion space

$$
\mathcal{G}_{d}-\mathcal{G}_{c}=-\left\langle 0\left|M_{j^{\prime}}^{i^{\prime}} M_{l^{\prime}}^{k^{\prime}} \operatorname{Tr}\left(M^{\dagger 2} M^{2}\right) M_{j}^{\dagger i} M_{l}^{\dagger k}\right| 0\right\rangle
$$

where now the $M^{i}{ }_{j}$ operators satisfy an anticommutation relation

$$
\left\{M_{j}^{i}, M_{l}^{\dagger k}\right\}=\delta_{j}^{k} \delta_{l}^{i}-\frac{1}{2 N_{F}} \delta_{j}^{i} \delta_{l}^{k}
$$

and Eq. (27) still holds. One can follow the same techniques as those outlined above for the $\hat{\mathcal{G}}_{+}$operators, using e.g. the states

$$
|5\rangle=M_{b}^{\dagger 1} M_{2}^{\dagger b}|0\rangle, \quad|6\rangle=M_{3}^{\dagger 1} M_{3}^{\dagger 2}|0\rangle, \quad|7\rangle=M_{1}^{\dagger 3} M_{2}^{\dagger 3}|0\rangle
$$

for the $\mathbf{3 5} \mathbf{5}_{a}, \mathbf{2 8 0}$ and $\mathbf{2 8 0} *$ antisymmetric representations.

[1] S. Weinberg, Physica A 96, 327 (1979).

[2] J. Gasser and H. Leutwyler, Annals Phys. 158, 142 (1984).

[3] J. Gasser and H. Leutwyler, Nucl. Phys. B 250, 465 (1985).

[4] U. G. Meissner, Rept. Prog. Phys. 56, 903 (1993).

[5] V. Bernard, N. Kaiser and U. G. Meissner, Int. J. Mod. Phys. E 4, 193 (1995).

[6] A. Pich, Rept. Prog. Phys. 58, 563 (1995).

[7] G. Ecker, Prog. Part. Nucl. Phys. 35, 1 (1995).

[8] S. Scherer, Adv. Nucl. Phys. 27, 277 (2003).

[9] V. Bernard, Prog. Part. Nucl. Phys. 60, 82 (2008).

[10] S. Scherer, Prog. Part. Nucl. Phys. 64, 1 (2010)

[11] T. N. Truong, Phys. Rev. Lett. 61, 2526 (1988).

[12] A. Dobado, M. J. Herrero and T. N. Truong, Phys. Lett. B 235, 134 (1990).

[13] A. Dobado and J. R. Pelaez, Phys. Rev. D 47, 4883 (1993).

[14] N. Kaiser, P. B. Siegel and W. Weise, Nucl. Phys. A 594, 325 (1995).

[15] A. Dobado and J. R. Pelaez, Phys. Rev. D 56, 3057 (1997).

[16] T. Hannah, Phys. Rev. D 55, 5613 (1997).

[17] E. Oset and A. Ramos, Nucl. Phys. A 635, 99 (1998).

[18] J. A. Oller, E. Oset and J. R. Pelaez, Phys. Rev. Lett. 80, 3452 (1998).

[19] J. A. Oller and E. Oset, Nucl. Phys. A 620, 438 (1997) [Erratum-ibid. A 652, 407 (1999)].

[20] J. Nieves and E. Ruiz Arriola, Phys. Lett. B 455, 30 (1999).

[21] N. Kaiser, Eur. Phys. J. A 3, 307 (1998).

[22] J. A. Oller, E. Oset and J. R. Pelaez, Phys. Rev. D 59, 074001 (1999) [Erratum-ibid. D 60, 099906 (1999); Erratum-ibid. D75, 099903 (2007)]. 
[23] J. A. Oller and E. Oset, Phys. Rev. D 60 (1999) 074023 arXiv:hep-ph/9809337.

[24] J. Nieves and E. Ruiz Arriola, Nucl. Phys. A 679, 57 (2000).

[25] A. Gomez Nicola, J. Nieves, J. R. Pelaez and E. Ruiz Arriola, Phys. Lett. B 486, 77 (2000).

[26] J. Nieves and E. Ruiz Arriola, Phys. Rev. D 63, 076001 (2001).

[27] V. E. Markushin, Eur. Phys. J. A 8, 389 (2000).

[28] J. A. Oller and U. G. Meissner, Phys. Lett. B 500, 263 (2001).

[29] J. Nieves, M. Pavon Valderrama and E. Ruiz Arriola, Phys. Rev. D 65, 036002 (2002).

[30] A. Gomez Nicola and J. R. Pelaez, Phys. Rev. D 65, 054009 (2002).

[31] M. F. M. Lutz and E. E. Kolomeitsev, Nucl. Phys. A 700, 193 (2002).

[32] J. Nieves and E. Ruiz Arriola, Phys. Rev. D 64, 116008 (2001).

[33] T. Hyodo, S. I. Nam, D. Jido and A. Hosaka, Phys. Rev. C 68, 018201 (2003).

[34] C. Garcia-Recio, J. Nieves, E. Ruiz Arriola and M. J. Vicente Vacas, Phys. Rev. D 67, 076009 (2003).

[35] E. E. Kolomeitsev and M. F. M. Lutz, Phys. Lett. B 585, 243 (2004).

[36] C. Garcia-Recio, M. F. M. Lutz and J. Nieves, Phys. Lett. B 582, 49 (2004).

[37] M. F. M. Lutz and E. E. Kolomeitsev, Nucl. Phys. A 730, 392 (2004).

[38] D. Jido, J. A. Oller, E. Oset, A. Ramos and U. G. Meissner, Nucl. Phys. A 725, 181 (2003).

[39] A. G. Nicola, J. Nieves, J. R. Pelaez and E. R. Arriola, Phys. Rev. D 69, 076007 (2004).

[40] S. Sarkar, E. Oset and M. J. Vicente Vacas, Nucl. Phys. A 750, 294 (2005) [Erratum-ibid. A 780, 78 (2006)].

[41] C. Garcia-Recio, J. Nieves and L. L. Salcedo, Phys. Rev. D 74, 034025 (2006).

[42] B. Borasoy, R. Nissler and W. Weise, Eur. Phys. J. A 25, 79 (2005).

[43] L. Roca, E. Oset and J. Singh, Phys. Rev. D 72, 014002 (2005).

[44] C. Garcia-Recio, J. Nieves and L. L. Salcedo, Eur. Phys. J. A 31, 499 (2007).

[45] A. Gomez Nicola, J. R. Pelaez and G. Rios, Phys. Rev. D 77, 056006 (2008).

[46] H. Toki, C. Garcia-Recio and J. Nieves, Phys. Rev. D 77, 034001 (2008).

[47] J. R. Pelaez, Phys. Rev. Lett. 92, 102001 (2004).

[48] J. R. Pelaez and G. Rios, Phys. Rev. Lett. 97, 242002 (2006).

[49] C. Garcia-Recio, J. Nieves and L. L. Salcedo, Phys. Rev. D 74, 036004 (2006).

[50] T. Hyodo, D. Jido and L. Roca, Phys. Rev. D 77, 056010 (2008).

[51] L. Roca, T. Hyodo and D. Jido, Nucl. Phys. A 809, 65 (2008).

[52] L. S. Geng, E. Oset, J. R. Pelaez and L. Roca, Eur. Phys. J. A 39, 81 (2009).

[53] J. Nieves and E. Ruiz Arriola, Phys. Rev. D 80, 045023 (2009).

[54] J. Nieves and E. Ruiz Arriola, Phys. Lett. B 679, 449 (2009).

[55] A. Martinez Torres, K. P. Khemchandani and E. Oset, Phys. Rev. C 77, 042203(R) (2008).

[56] A. Martinez Torres, K. P. Khemchandani, L. S. Geng, M. Napsuciale and E. Oset, Phys. Rev. D 78, 074031 (2008).

[57] E. E. Kolomeitsev and M. F. M. Lutz, Phys. Lett. B 582, 39 (2004).

[58] J. Hofmann and M. F. M. Lutz, Nucl. Phys. A 733, 142 (2004).

[59] F. K. Guo, P. N. Shen, H. C. Chiang and R. G. Ping, Phys. Lett. B 641, 278 (2006).

[60] D. Gamermann, E. Oset, D. Strottman and M. J. Vicente Vacas, Phys. Rev. D 76, 074016 (2007).

[61] M. F. M. Lutz and E. E. Kolomeitsev, Nucl. Phys. A 730, 110 (2004).

[62] J. Hofmann and M. F. M. Lutz, Nucl. Phys. A 763, 90 (2005).

[63] J. Hofmann and M. F. M. Lutz, Nucl. Phys. A 776, 17 (2006).

[64] T. Mizutani and A. Ramos, Phys. Rev. C 74, 065201 (2006).

[65] C. Garcia-Recio, V. K. Magas, T. Mizutani, J. Nieves, A. Ramos, L. L. Salcedo and L. Tolos, Phys. Rev. D 79, 054004 (2009).

[66] D. Gamermann, C. Garcia-Recio, J. Nieves, L. L. Salcedo and L. Tolos, Phys. Rev. D in press and arXiv:1002.2763

[67] S. Weinberg, Phys. Rev. Lett. 17, 616 (1966).

[68] Y. Tomozawa, Nuovo Cim. 46A, 707 (1966).

[69] R. Molina, D. Nicmorus and E. Oset, Phys. Rev. D 78, 114018 (2008).

[70] L. S. Geng and E. Oset, Phys. Rev. D 79, 074009 (2009); L. S. Geng, E. Oset, R. Molina and D. Nicmorus, arXiv:0905.0419 [hep-ph].

[71] E. Oset and A. Ramos, arXiv:0905.0973 [hep-ph].

[72] P. Gonzalez, E. Oset and J. Vijande, Phys. Rev. C 79, 025209 (2009).

[73] S. Sarkar, B. X. Sun, E. Oset and M. J. V. Vacas, arXiv:0902.3150 [hep-ph].

[74] M. Bando, T. Kugo, S. Uehara, K. Yamawaki and T. Yanagida, Phys. Rev. Lett. 541215 (1985).

[75] M. Bando, T. Kugo and K. Yamawaki, Phys. Rept. 164217 (1988).

[76] A. Martinez Torres, L. S. Geng, L. R. Dai, B. X. Sun, E. Oset and B. S. Zou, Phys. Lett. B 680,310 (2009).

[77] T. Branz, L. S. Geng and E. Oset, Phys. Rev. D 81, 054037 (2010).

[78] L. S. Geng, F. K. Guo, C. Hanhart, R. Molina, E. Oset and B. S. Zou, Eur. Phys. J. A 44, 305 (2009).

[79] D. G. Caldi and H. Pagels, Phys. Rev. D 14, 809 (1976).

[80] D. G. Caldi and H. Pagels, Phys. Rev. D 15, 2668 (1977).

[81] L. E. Ibanez, Phys. Lett. B 86, 340 (1979).

[82] U. G. Meissner and V. Pasquier, Phys. Lett. B 235, 153 (1990).

[83] J. Smit, Nucl. Phys. B 175, 307 (1980).

[84] C. Amsler et al. [Particle Data Group], Phys. Lett. B 667, 1 (2008). 
[85] M. C. Birse, Z. Phys. A 355, 231 (1996).

[86] T. Hyodo, D. Jido and A. Hosaka, Phys. Rev. D 75, 034002 (2007) arXiv:hep-ph/0611004.

[87] F. Gursey and L. A. Radicati, Phys. Rev. Lett. 13, 173 (1964).

[88] A. Pais, Phys. Rev. Lett. 13, 175 (1964).

[89] B. Sakita, Phys. Rev. 136, B1756 (1964).

[90] J. J. de Swart, Rev. Mod. Phys. 35916 (1963) [Erratum-ibid. 37326 (1965)].

[91] C. L. Cook and G. Murtaza, Nuovo Cim. 39531 (1965).

[92] R. P. Feynman, M. Gell-Mann and G. Zweig, Phys. Rev. Lett. 13, 678 (1964).

[93] S. R. Coleman and J. Mandula, Phys. Rev. 159, 1251 (1967).

[94] G. Ecker, J. Gasser, A. Pich and E. de Rafael, Nucl. Phys. B 321, 311 (1989).

[95] G. Ecker, J. Gasser, H. Leutwyler, A. Pich and E. de Rafael, Phys. Lett. B 223, 425 (1989).

[96] M. Jaminon and E. Ruiz Arriola, Phys. Lett. B 443, 33 (1998).

[97] S. Weinberg, Phys. Rev. 166, 1568 (1968).

[98] D. G. Caldi,

[99] A. V. Manohar and M. B. Wise, Camb. Monogr. Part. Phys. Nucl. Phys. Cosmol. 10, 1 (2000).

[101] H. Nagahiro, L. Roca, A. Hosaka and E. Oset, Phys. Rev. D 79, 014015 (2009).

[102] F. E. Close and Q. Zhao, Phys. Rev. D 71, 094022 (2005).

[103] C. Amsler and F. E. Close, Phys. Rev. D 53, 295 (1996).

[104] C. Amsler and N. A. Tornqvist, Phys. Rept. 389, 61 (2004).

[105] M. Albaladejo and J. A. Oller, Phys. Rev. Lett. 101, 252002 (2008).

[106] A. V. Anisovich et al. [Crystal Barrel Collaboration], Phys. Lett. B 452, 173 (1999).

[107] A. V. Anisovich et al., Phys. Lett. B 542, 8 (2002).

[108] S. N. Cherry and M. R. Pennington, Nucl. Phys. A 688, 823 (2001).

[109] L. S. Geng, E. Oset, L. Roca and J. A. Oller, Phys. Rev. D 75, 014017 (2007).

[110] C. Daum et al. [ACCMOR Collaboration], Nucl. Phys. B 187, 1 (1981).

[111] A. Filippi et al. [OBELIX Collaboration], Phys. Lett. B 495, 284 (2000).

[112] S. Ishida, M. Oda, H. Sawazaki and K. Yamada, Prog. Theor. Phys. 82, 119 (1989). 